\title{
Search for new phenomena with large jet multiplicities and missing transverse momentum using large-radius jets and flavour-tagging at ATLAS in $13 \mathrm{TeV} p p$ collisions
}

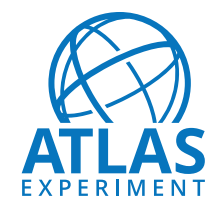

\section{The ATLAS collaboration}

E-mail: atlas.publications@cern.ch

AbStRact: A search is presented for particles that decay producing a large jet multiplicity and invisible particles. The event selection applies a veto on the presence of isolated electrons or muons and additional requirements on the number of $b$-tagged jets and the scalar sum of masses of large-radius jets. Having explored the full ATLAS 2015-2016 dataset of LHC proton-proton collisions at $\sqrt{s}=13 \mathrm{TeV}$, which corresponds to $36.1 \mathrm{fb}^{-1}$ of integrated luminosity, no evidence is found for physics beyond the Standard Model. The results are interpreted in the context of simplified models inspired by R-parity-conserving and R-parity-violating supersymmetry, where gluinos are pair-produced. More generic models within the phenomenological minimal supersymmetric Standard Model are also considered.

Keywords: Beyond Standard Model, Hadron-Hadron scattering (experiments), Supersymmetry

ARXiv EPRINT: 1708.02794 


\section{Contents}

1 Introduction 1

2 ATLAS detector 2

3 Collision data and simulated event samples 3

3.1 Data 3

3.2 Simulated event samples 3

3.2.1 Background process simulation 3

3.2.2 Supersymmetric signal models 5

4 Event reconstruction $\quad 7$

4.1 Primary vertex $\quad 7$

4.2 Jets 7

4.3 Electrons and photons 8

4.4 Muons 8

4.5 Overlap removal 9

4.6 Missing transverse momentum 9

5 Event selection $\quad 9$

5.1 Signal region definitions 10

5.1.1 Heavy-flavour channel 10

5.1.2 Jet mass channel 10

5.2 Control region definitions 11

$\begin{array}{lll}\text { 5.2.1 Multijet template region } & 12\end{array}$

$\begin{array}{ll}5.2 .2 & \text { Leptonic control regions } \\ \end{array}$

$\begin{array}{lll}6 & \text { Background estimation techniques } & 13\end{array}$

6.1 Multijet template estimation 13

$\begin{array}{ll}6.2 \text { Leptonic background estimates } & 15\end{array}$

$\begin{array}{lll}6.3 & \text { Combined background fits } & 16\end{array}$

$\begin{array}{lll}7 & \text { Statistical procedures } & 18\end{array}$

$\begin{array}{lll}7.1 & \text { Systematic uncertainties } & 18\end{array}$

$\begin{array}{lll}7.2 & \text { Hypothesis testing } & 19\end{array}$

8 Results and interpretation $\quad 19$

8.1 Exclusion limits 20

9 Conclusion $\quad 26$

The ATLAS collaboration $\quad 34$ 


\section{Introduction}

As the experiments at the Large Hadron Collider [1] (LHC) continue to amass data from the $13 \mathrm{TeV}$ centre-of-mass energy run, observing the production of heavy resonances remains a principal path in the search for physics beyond the Standard Model (SM), such as supersymmetry (SUSY) [2-7]. One distinctive signature of such processes would be an increased incidence of events containing a large number of jets accompanied by missing transverse momentum (the magnitude of which is denoted $E_{\mathrm{T}}^{\mathrm{miss}}$ ). These could originate from extended cascade decays of heavy particles through lighter states, which might interact weakly and therefore have remained unobserved due to their low production cross-sections.

A particle spectrum of this nature is exemplified by the pair production of heavy gluinos $(\tilde{g})$ that decay via long cascade chains, such as through the superpartners of the electroweak and Higgs bosons. In R-parity-conserving (RPC) [8] SUSY models, these decays culminate in the production of a stable lightest supersymmetric particle (LSP). Cosmological and other observations prohibit an electrically charged or strongly interacting LSP [9-12], hence the production of these objects, invisible to the detector, would result in missing transverse momentum. Similarly, large jet multiplicities could also be achieved if the gluinos were to decay via on- or off-shell top squarks $\left(\tilde{t}_{1}\right)$ or via $\mathrm{R}$-parity-violating (RPV) [13] couplings. In the latter case, the LSP could decay within the detector volume, softening the $E_{\mathrm{T}}^{\text {miss }}$ spectrum.

This paper reports the results of an analysis of $36.1 \mathrm{fb}^{-1}$ of proton-proton $(p p)$ collision data recorded at $\sqrt{s}=13 \mathrm{TeV}$ by the ATLAS experiment [14] in 2015 and 2016, scrutinising events that contain significant $E_{\mathrm{T}}^{\mathrm{miss}}$ and at least seven jets with a large transverse momentum $\left(p_{\mathrm{T}}\right)$. Selected events are further classified based on the presence of jets containing $B$-hadrons ( $b$-jets) or on the sum of the masses of large-radius jets. The $b$-jet selection improves the sensitivity to beyond-the-SM (BSM) signals with enhanced heavyflavour decays. Given the unusually high jet multiplicities of the target signatures, large jet masses can originate both from capturing the decay products from boosted heavy particles including top quarks and from accidental combinations [15]. A key feature of the search is the data-driven method used to estimate the dominant background from multijet production. Other major background processes include top quark pair production $(t \bar{t})$ and $W$ boson production in conjunction with jets $(W+$ jets $)$.

Searches by ATLAS were previously reported using smaller quantities of LHC data taken at $\sqrt{s}=7$ and $8 \mathrm{TeV}$ from $2011-2012$ [16-18] and at $\sqrt{s}=13 \mathrm{TeV}$ in 2015 [19]. Due to the more modest selection on $E_{\mathrm{T}}^{\text {miss }}$, the analysis reported in this paper is sensitive to classes of signals not excluded by related searches performed by ATLAS [20-24] and CMS [25-35].

In the next section the ATLAS detector is described, followed by a description of the accumulated data and simulated event samples in section 3. Then the event reconstruction and selection are explained in sections 4 and 5. The data-driven method to estimate the multijet background and the estimation of systematic errors are in sections 6 and 7 . The result and interpretations are presented in section 8 followed by conclusions in section 9 . 


\section{ATLAS detector}

The ATLAS detector [14] at the LHC covers nearly the entire solid angle ${ }^{1}$ around the collision point. It consists of an inner tracking detector surrounded by a thin superconducting solenoid, electromagnetic and hadronic calorimeters, and a muon spectrometer incorporating three large superconducting toroid magnets. The inner-detector system (ID) is immersed in a $2 \mathrm{~T}$ axial magnetic field and provides charged-particle tracking in the range $|\eta|<2.5$.

The high-granularity silicon pixel detector covers the vertex region and typically provides four measurements per track. It is followed by the silicon microstrip tracker which usually provides four two-dimensional measurement points per track. These silicon detectors are complemented by the transition radiation tracker, which enables the radial extension of tracks with $|\eta|<2.0$. The transition radiation tracker (TRT) also provides electron identification information based on the fraction of hits (typically 30 in total) above a higher energy-deposit threshold corresponding to the emission of transition radiation.

The calorimeter system covers the pseudorapidity range $|\eta|<4.9$. Within the region $|\eta|<3.2$, electromagnetic calorimetry is provided by barrel and endcap high-granularity lead/liquid-argon (LAr) electromagnetic calorimeters, with an additional thin LAr presampler covering $|\eta|<1.8$, to correct for energy loss in material upstream of the calorimeters. Hadronic calorimetry is provided by the steel/scintillating-tile calorimeter, segmented into three barrel structures within $|\eta|<1.7$, and two copper/LAr hadronic endcap calorimeters. The solid angle coverage is completed with forward copper/LAr and tungsten/LAr calorimeter modules optimised for electromagnetic and hadronic measurements respectively.

The muon spectrometer (MS) comprises separate trigger and high-precision tracking chambers measuring the deflection of muons in a magnetic field generated by superconducting air-core toroids. The precision chamber system covers the region $|\eta|<2.7$ with three layers of monitored drift tubes, complemented by cathode strip chambers in the forward region, where the background is highest. The muon trigger system covers the range $|\eta|<2.4$ with resistive plate chambers in the barrel, and thin gap chambers in the endcap regions.

A two-level trigger system is used to select interesting events [36, 37]. The Level-1 trigger is implemented in low-latency electronics and uses a subset of detector information to reduce the event rate to below $90 \mathrm{kHz}$. This is followed by a software-based High-Level Trigger which reduces the average event rate to about $1 \mathrm{kHz}$.

\footnotetext{
${ }^{1}$ ATLAS uses a right-handed coordinate system with its origin at the nominal interaction point (IP) in the centre of the detector and the $z$-axis along the beam pipe. The $x$-axis points from the IP to the centre of the LHC ring, and the $y$-axis points upwards. Cylindrical coordinates $(r, \phi)$ are used in the transverse plane, $\phi$ being the azimuthal angle around the $z$-axis. The pseudorapidity is defined in terms of the polar angle $\theta$ as $\eta=-\ln \tan (\theta / 2)$. Angular distance is measured either in units of $\Delta R_{\mathrm{y}} \equiv \sqrt{(\Delta \mathrm{y})^{2}+(\Delta \phi)^{2}}$, where $\mathrm{y}$ is the rapidity $1 / 2 \ln \left(\left(E+p_{z}\right) /\left(E-p_{z}\right)\right)$, or in units of $\Delta R$ which is the corresponding quantity in which the pseudorapidity replaces the rapidity.
} 


\section{Collision data and simulated event samples}

Data recorded by ATLAS during 2015 and 2016 are used in this analysis for background estimation as well as in the final signal region (SR) selections. Simulated events produced with several Monte Carlo (MC) event generators provide predictions for subdominant background contributions from SM processes producing top quarks and vector bosons. The main source of background is multijet production, for which predictions are derived directly from data, as described in section 6.1. Models of potential signals are likewise simulated for analysis optimisation and interpretation of the final results.

\subsection{Data}

Collision events studied for this paper comprise $3.21 \pm 0.07 \mathrm{fb}^{-1}$ recorded with good data quality in 2015 with a further $32.9 \pm 1.1 \mathrm{fb}^{-1}$ recorded during 2016, all with a bunch spacing of $25 \mathrm{~ns}$. The luminosity uncertainty was derived using beam-separation scans, following a methodology similar to that detailed in ref. [38]. Pile-up, i.e. additional $p p$ interactions in the same or adjacent bunch crossings, contribute to the signals registered by the detector. For this dataset, the average number of interactions per bunch crossing ranged up to 52, with a mean of 22.9 .

Events were recorded with a variety of triggers. During both 2015 and 2016, events were selected by a trigger requiring at least six jets with $p_{\mathrm{T}}>45 \mathrm{GeV}$ and central pseudorapidity, $|\eta|<2.4$. In addition, in 2015 events were triggered by requiring the presence of at least five jets with $p_{\mathrm{T}}>70 \mathrm{GeV}$, and in 2016 with a trigger requiring at least five jets with $p_{\mathrm{T}}>65 \mathrm{GeV}$ and $|\eta|<2.4$.

Minimum data quality requirements are imposed to ensure that only events are used in which the entire ATLAS detector was functioning well. These, for example, exclude data corruption in the ID and calorimeters, excessive noise and spurious jets produced by non-collision backgrounds [39, 40].

\subsection{Simulated event samples}

All simulated events were overlaid with multiple $p p$ collisions simulated with the soft QCD processes of Pythia 8.186 [41] using the A2 set of parameters (A2 tune) [42] and the MSTW2008LO parton distribution functions (PDFs) [43]. The simulated events were required to pass the trigger, and were weighted such that the pile-up conditions match those of the data. The response of the detector to particles was modelled with an ATLAS detector simulation [44] based fully on GEANT4 [45], or using fast simulation based on a parameterisation of the performance of the ATLAS electromagnetic and hadronic calorimeters [46] and on GEANT4 elsewhere.

\subsubsection{Background process simulation}

For the generation of $t \bar{t}$ and single top quarks in the $W t$ - and $s$-channels PowheGBox v2 [47-52] was used with the CT10 PDF sets [53] in the matrix element calculations. Electroweak $t$-channel single-top-quark events were generated with PowHEG-Box v1, using the four-flavour scheme for the next-to-leading-order (NLO) matrix element calculations, 
together with the fixed four-flavour PDF set CT10f4 [53], and with top quark decays using MADSpIn [54], preserving all spin correlations. The parton shower, fragmentation, and the underlying event were simulated using PYTHIA v6.428 [55] with the CTEQ6L1 PDF sets [56] and the Perugia 2012 tune (P2012) [57]. The top quark mass was set to $172.5 \mathrm{GeV}$. The EvtGen v1.2.0 program [58] was used to model the properties of the bottom and charm hadron decays. Simulated $t \bar{t}$ events were normalised to the cross-section calculated to nextto-next-to-leading order (NNLO) in perturbative QCD, including soft-gluon resummation to next-to-next-to-leading-logarithm (NNLL) order [59].

Events containing $t \bar{t}$ and additional heavy particles - comprising three-top, four-top, $t \bar{t}+W, t \bar{t}+Z$ and $t \bar{t}+W W$ production - were simulated at leading order (LO) in the strong coupling constant $\alpha_{\mathrm{S}}$, using MADGRAPH5 v2.2.2 [60] with up to two additional partons in the matrix element, interfaced to the PyтнiA 8.186 parton shower model. The A14 set of Pythia 8 parameters was used [61], together with the NNPDF2.3 LO PDF set [62]. The predicted production cross-sections were calculated to NLO as described in ref. [60]. In addition, $t \bar{t}+H$ events were simulated at NLO using MADGRAPH5_AMC@NLO v2.2.3 [60], with the NNPDF3.0 NLO PDF set [63] used in the matrix element calculation, and again interfaced to PYTHIA 8.186 for the parton shower, with the A14 tune and the NNPDF2.3 LO PDFs.

Events containing a $W$ or $Z$ bosons associated with jets were simulated using the ShERPA 2.2.1 generator. Matrix elements were calculated for up to two partons at NLO and four partons at LO using the Comix [64] and OpenLoops [65] matrix element generators and merged with the SHERPA parton shower [66] using the ME+PS@NLO prescription [67]. The NNPDF3.0 NNLO PDF set was used in association with a tuning performed by the SHERPA authors.

Diboson processes with four charged leptons, three charged leptons + one neutrino, or two charged leptons + two neutrinos, were simulated using Sherpa v2.1.1 [68]. The matrix element calculations contained all diagrams with four electroweak vertices. They were calculated for up to one (for $4 \ell, 2 \ell+2 \nu$ ) or zero additional partons (for $3 \ell+1 \nu$ ) at NLO and up to three additional partons at LO using the Comix and OpenLoops matrix element generators and merged with the SHERPA parton shower using the ME+PS@NLO prescription. The CT10 PDF set was used in conjunction with dedicated parton shower tuning developed by the SHERPA authors. An identical procedure was followed to simulate diboson production with one hadronically decaying boson accompanied by one charged lepton and one neutrino, two charged leptons or two neutrinos, where the calculations included one additional parton at NLO for $Z Z \rightarrow 2 \ell+q \bar{q}$ and $Z Z \rightarrow 2 \nu+q \bar{q}$ only, and up to three additional partons at LO.

Theoretical uncertainties were considered for all these simulated samples. By far the most important process simulated in this analysis is $t \bar{t}$ production, and several samples are compared to estimate the uncertainty in this background. Samples were produced with the factorisation and renormalisation scales varied coherently, along with variations of the $h_{\text {damp }}$ parameter in PowHEG-Box and with parameters set for more/less radiation in the parton shower [69]. Additionally, to account for uncertainties from the parton shower modelling and generator choice, the nominal sample was compared to samples generated 
with Powheg-Box and MadGraph5_AMC@NLO, interfaced to Herwig++ [70]. The comparison with samples which vary the amount of additional radiation contributes the largest uncertainty in the signal region predictions.

Full simulation was used for all background MC samples, ensuring an accurate representation of detector effects. Further details of samples can be found in refs. [69, 71-74].

\subsubsection{Supersymmetric signal models}

A number of supersymmetric signal model samples are simulated to permit the interpretation of the search results in terms of supersymmetric parameters. Substantial cross-sections are possible for production of gluinos, superpartners of the gluon, whose cascade decays result in a large multiplicity of jets, which may also exhibit an unusually high heavy-flavour content or atypically large masses.

The first is a simplified model, in which gluinos are pair-produced and then decay through an off-shell squark via the cascade:

$$
\begin{aligned}
\tilde{g} & \rightarrow q+\bar{q}^{\prime}+\tilde{\chi}_{1}^{ \pm} \quad(q=u, d, s, c), \\
\tilde{\chi}_{1}^{ \pm} & \rightarrow W^{ \pm}+\tilde{\chi}_{2}^{0} \\
\tilde{\chi}_{2}^{0} & \rightarrow Z+\tilde{\chi}_{1}^{0},
\end{aligned}
$$

where the quarks are only permitted to be from the first two generations. The parameters of the model are the masses of the gluino, $m_{\tilde{g}}$, and the lightest neutralino, $m_{\tilde{\chi}_{1}^{0}}$. The mass of the $\tilde{\chi}_{1}^{ \pm}$is constrained to be $\left(m_{\tilde{g}}+m_{\tilde{\chi}_{1}^{0}}\right) / 2$, and the mass of the $\tilde{\chi}_{2}^{0}$ is set to $\left(m_{\tilde{\chi}_{1}^{ \pm}}+m_{\tilde{\chi}_{1}^{0}}\right) / 2$. A diagram of this "two-step" simplified model is shown in figure 1(a).

A second type of SUSY model is drawn from a two-dimensional subspace of the 19parameter phenomenological minimal supersymmetric Standard Model (pMSSM) [75, 76], motivated in part by models not previously excluded by the analysis of ref. [21]. An example pMSSM process is shown in figure 1(b). These models are selected to have a bino-dominated neutralino $\tilde{\chi}_{1}^{0}$, kinematically accessible gluinos, and an intermediate-mass Higgsino-dominated multiplet, containing two neutralinos (the $\tilde{\chi}_{2}^{0}$ and $\tilde{\chi}_{3}^{0}$ ) and a chargino (the $\tilde{\chi}_{1}^{ \pm}$). The masses of these particles are varied by changing the SUSY soft-breaking parameters $M_{3}$ (for the gluino) and $\mu$ (for the Higgsinos), while $M_{1}$ (for the $\tilde{\chi}_{1}^{0}$ ) is held constant at $60 \mathrm{GeV}$. In order that other SUSY particles remain kinematically inaccessible, the other parameters, defined in ref. [21], are set to $m_{A}=M_{2}=3 \mathrm{TeV}, A_{\tau}=0, \tan \beta=10$, $A_{t}=A_{b}=m_{(\tilde{e}, \tilde{\mu}, \tilde{\tau}) \mathrm{L}}=m_{(\tilde{e}, \tilde{\mu}, \tilde{\tau}) \mathrm{R}}=m_{\tilde{q} \mathrm{~L}(1,2,3)}=m_{(\tilde{u}, \tilde{c}, \tilde{t}) \mathrm{R}}=m_{(\tilde{d}, \tilde{s}, \tilde{b}) \mathrm{R}}=5 \mathrm{TeV}$. These values ensure theoretically consistent mass spectra, and produce a mass for the lightest scalar Higgs boson close to $125 \mathrm{GeV}$. Mass spectra with consistent electroweak symmetry breaking are generated using SoftSusy 3.4.0 [77]. The decay branching ratios are calculated with SDECAY/HDeCAY 1.3b/3.4 [78], and when $m_{\tilde{\chi}_{1}^{ \pm}} \lesssim 500 \mathrm{GeV}$ and $m_{\tilde{g}} \gtrsim 1200 \mathrm{GeV}$ the predominant decays are $\tilde{g} \rightarrow t+\bar{t}+\tilde{\chi}_{2}^{0}\left(\tilde{\chi}_{3}^{0}\right)$ and $\tilde{g} \rightarrow t+\bar{b}+\tilde{\chi}_{1}^{ \pm}$, with $\tilde{\chi}_{2}^{0}\left(\tilde{\chi}_{3}^{0}\right)$ decaying to $Z / h+\tilde{\chi}_{1}^{0}$ and $\tilde{\chi}_{1}^{ \pm}$to $W^{ \pm}+\tilde{\chi}_{1}^{0}$. When these decays dominate, they lead to final states with many jets, several of which are $b$-jets, but relatively little $E_{\mathrm{T}}^{\text {miss }}$. This renders this search particularly sensitive compared to most other SUSY searches which tend to require 


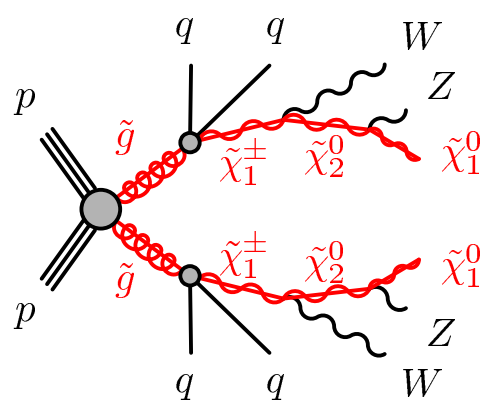

(a) Two-step decay

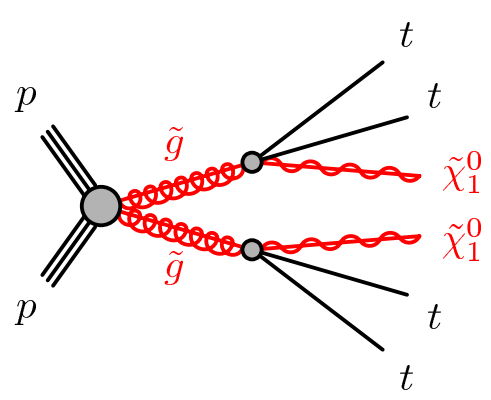

(c) Off-shell top squarks

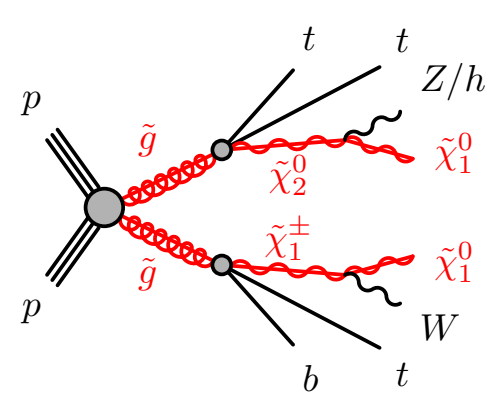

(b) pMSSM

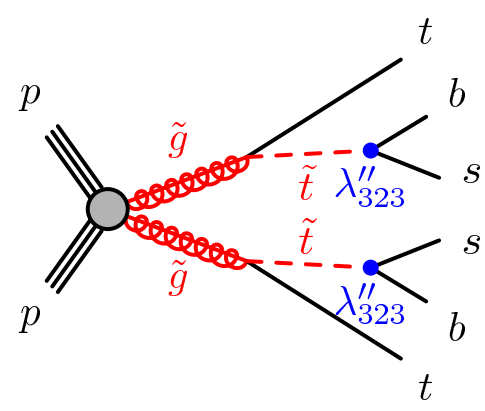

(d) RPV

Figure 1. Pseudo-Feynman diagrams for the different signal models used in this search.

high $E_{\mathrm{T}}^{\text {miss }}$. At higher $m_{\tilde{\chi}_{1}^{ \pm}}$and lower $m_{\tilde{g}}$, the decay $\tilde{g} \rightarrow q q \tilde{\chi}_{1}^{0}$ becomes dominant and this search starts to lose sensitivity. This model is labelled in the following figures as 'pMSSM'.

Gluino-mediated top squark $\left(\tilde{t}_{1}\right)$ production, with the squarks being off-shell, is also a good match for the target final state. This scenario is characterised by the pair-production of gluinos followed by their decay with $100 \%$ branching ratio to $t \bar{t}+\tilde{\chi}_{1}^{0}$, through a virtual top squark. Naturalness arguments for supersymmetry favour light gluinos, top squark, and Higgsinos, so this final state is very well motivated. Figure 1(c) shows a diagram for the off-shell process.

Permitting non-zero $R$-parity-violating (RPV) couplings allows consideration of another variety of gluino-mediated top squark production, wherein the last step of the decay proceeds via a baryon-number-violating interaction: $\tilde{t}_{1} \rightarrow \bar{s}+\bar{b}$ (charge conjugates implied). Figure 1(d) presents the RPV model. Such $R$-parity-violating models give rise to final states with low missing transverse momentum. Uniquely among the searches for strongly-produced supersymmetric particles, the current analysis selects final states with sufficiently low missing transverse momentum to be sensitive to these RPV scenarios.

The signal samples were generated using MADGRAPH5_AMC@NLO v2.2.2 interfaced to Pythia 8.186 with the A14 tune for the modelling of the parton shower (PS), hadronisation and underlying event. The matrix element (ME) calculation was performed at tree level and includes the emission of up to two additional partons. The PDF set used for the generation was NNPDF23LO. The ME-to-PS matching was done using the CKKW-L prescription [79], with a matching scale set to $m_{\tilde{g}} / 4$. 
The gluino-mediated top squark production signal samples were generated with full simulation of the ATLAS detector, whereas the other signal MC samples employed the fast detector simulation.

Signal cross-sections are calculated to NLO in the strong coupling constant, adding the resummation of soft gluon emission at next-to-leading-logarithm accuracy $(\mathrm{NLO}+\mathrm{NLL})$ [80-84]. The nominal cross-section and the uncertainty are taken from an envelope of cross-section predictions using different PDF sets and factorisation and renormalisation scales, as described in ref. [85].

\section{Event reconstruction}

\subsection{Primary vertex}

Primary vertices are reconstructed using at least two charged-particle tracks with $p_{\mathrm{T}}>$ $400 \mathrm{MeV}$ measured by the ID [86]. The primary vertex with the largest sum of squared track transverse momenta $\left(\sum p_{\mathrm{T}}^{2}\right)$ is designated as the hard scatter vertex.

\subsection{Jets}

Jets are reconstructed from three-dimensional topological clusters of calorimeter cells (topoclusters) that are noise-suppressed and calibrated to the electromagnetic scale, i.e. corrected for the calorimeter response to electrons and photons [87]. Small-radius jets are built by applying the anti- $k_{t}$ clustering algorithm [88], as implemented in FastJet [89], with jet radius parameter $R=0.4$, to the topoclusters. Four-momentum corrections are applied to the jets, starting with a subtraction procedure that removes the average estimated energy contributed by pile-up interactions based on the jet area [90]. This is followed by jet energy scale calibrations that restore the jet energy to the mean response versus particle-level simulation, using a global sequential calibration to correct finer variations due to flavour and detector geometry and finally in situ corrections that match the data to the MC scale [91]. Only jets with $p_{\mathrm{T}}>20 \mathrm{GeV}$ and $|\eta|<2.8$ are considered, with the exception of the $E_{\mathrm{T}}^{\text {miss }}$ calculation, for which jets in the range $2.8 \leq|\eta|<4.5$ are also used (see section 4.6).

To eliminate jets containing a large energy contribution from pile-up, jets are tested for compatibility with the hard scatter vertex with the jet vertex tagger (JVT) discriminant, utilising information from the ID tracks associated with the jet [92]. Any jets with $20<$ $p_{\mathrm{T}}<60 \mathrm{GeV}$ and $|\eta|<2.4$ for which JVT $<0.59$ are considered to originate from pile-up and are therefore rejected from the analysis. Scale factors derived from data are applied for the simulated samples to correct the efficiency of the JVT selection.

A multivariate discriminant (MV2c10) is used to tag jets containing $B$-hadrons [93]. This exploits the long lifetime, high decay multiplicity, hard fragmentation and large mass of $B$-hadrons. The selected working point for the $b$-tagging algorithm [94] tags $b$-jets in the pseudorapidity range $|\eta|<2.5$ with an efficiency of approximately $70 \%$ in simulated $t \bar{t}$ events, and rejects $c$-jets, $\tau$-jets and light-quark or gluon jets by factors of $9.6,31$ and 254, respectively. For the purposes of overlap removal, a loose b-tag designation is 
defined using a working point with $80 \%$-tagging efficiency. Where $b$-tagging selections are applied, efficiency corrections measured in data are applied to simulated events, to improve modelling of the $b$-tagging efficiencies.

In a second jet formation step [95], small-radius jets with $p_{\mathrm{T}}>20 \mathrm{GeV}$ and $|\eta|<2.0$ are reclustered using the anti- $k_{t}$ algorithm with radius parameters $R=1.0$ to form largeradius jets. The input jets are required to pass an overlap removal procedure accounting for ambiguities between jets and leptons, as discussed below. In the leptonic control region (CR) defined in section 5.2, electrons and muons may also be included in the inputs to the jet reclustering provided they satisfy $p_{\mathrm{T}}>20 \mathrm{GeV}$ and $|\eta|<2.0$ as for standard jets. Large-radius jets are retained for analysis if they have $p_{\mathrm{T}}>100 \mathrm{GeV}$ and $|\eta|<1.5$.

\subsection{Electrons and photons}

Electron and photon candidates are reconstructed from clusters of calorimeter cells defined with fixed rectangular $\eta-\phi$ sizes and then distinguished by matching to ID tracks [96, 97]. A multivariate calibration is applied to correct the electron/photon energy scale [98].

Electron candidates are preselected if they have $p_{\mathrm{T}}>10 \mathrm{GeV},|\eta|<2.47$ and pass a "Loose" likelihood-based quality selection accounting for lateral shower shapes, hadronic shower leakage, ${ }^{2}$ hits on tracks, track-cluster matching and the number of high-threshold hits in the TRT. Signal electrons with $p_{\mathrm{T}}>20 \mathrm{GeV}$ are defined by requiring a "Tight" likelihood selection including impact parameter restrictions and the "GradientLoose" isolation requirement from ref. [96] in addition to the preselection. To achieve additional rejection of background electrons from non-prompt sources, signal electron tracks must be matched to the hard scatter vertex with longitudinal impact parameter $\left|z_{0}\right|<0.5 \mathrm{~mm}$ and transverse impact parameter significance $\left|d_{0}\right| / \sigma\left(d_{0}\right)<5$. Corrections to the electron reconstruction and identification efficiency in simulated samples are applied using scale factors measured in data [96].

Photon candidates likewise are identified using tight criteria defined by lateral shower shapes in the first and second layers of the electromagnetic calorimeter, as well as the degree of hadronic shower leakage. Acceptance requirements of $p_{\mathrm{T}}>40 \mathrm{GeV}$ and $|\eta|<2.37$ are applied.

\subsection{Muons}

Muon candidates are reconstructed from tracks formed in the ID and MS, which are combined for improved precision and background rejection [99]. Stand-alone muon tracks are used to extend the muon reconstruction coverage beyond the ID acceptance in pseudorapidity (from $|\eta|=2.5$ to $|\eta|=2.7$ ).

Preselected muons are defined by the "medium" selection of ref. [99] using the number of hits on track and track quality and compatibility between the ID and MS measurements. These must have $p_{\mathrm{T}}>10 \mathrm{GeV}$ and $|\eta|<2.7$. Signal muons must have a higher transverse momentum, $p_{\mathrm{T}}>20 \mathrm{GeV}$, and satisfy the "GradientLoose" isolation requirement [99], as well as impact parameter matching requirements similar to those for electrons:

\footnotetext{
${ }^{2}$ Energy measured in the hadronic calorimeter, within the cluster window.
} 
$\left|z_{0}\right|<0.5 \mathrm{~mm}$ and $\left|d_{0}\right| / \sigma\left(d_{0}\right)<3$. Muon reconstruction and identification efficiencies are corrected with scale factors in simulated samples [100].

\subsection{Overlap removal}

To avoid double counting, a procedure of overlap removal was applied to jets, photons and leptons as follows. The electrons and muons are those passing the preselection.

1. If an electron and a muon share an ID track, the electron is removed and the muon is retained.

2. Photons with $\Delta R_{y}<0.4$ relative to an electron or a muon are deselected.

3. Any jet that fails the loose $b$-tag selection is removed if either:

- it falls $\Delta R_{y}<0.2$ from an electron; or

- it has no more than three tracks with $p_{\mathrm{T}}>500 \mathrm{MeV}$, or contains an ID track matched to a muon such that $p_{\mathrm{T}}^{\text {jet }}<2 p_{\mathrm{T}}^{\mu}$ and the muon track has more than $70 \%$ of the sum of the transverse momenta of all tracks in the jet, such that the jet resembles radiation from the muon.

4. Any electrons or muons with $\Delta R_{y}<0.4$ from a surviving jet are eliminated.

5. Finally, jets that have $\Delta R_{y}<0.4$ from photons are removed.

\subsection{Missing transverse momentum}

The missing transverse momentum, $E_{\mathrm{T}}^{\text {miss }}$, is defined as the magnitude of the negative vector sum of the transverse momenta of preselected electrons and muons, photons and jets, to which is added a "soft term" that recovers the contributions from other low- $p_{\mathrm{T}}$ particles [101]. The soft term is constructed from all tracks that are not associated with any of the preceding objects, and that are associated with the primary vertex. In this way, the missing transverse momentum is adjusted for the best calibration of the leptons, photons and jets, while maintaining pile-up independence in the soft term.

\section{Event selection}

Target signal events for this analysis are characterised by a large jet multiplicity, beyond what is generated by high-cross-section SM processes, combined with a $E_{\mathrm{T}}^{\text {miss }}$ that is significantly larger than that expected purely from detector resolution. Several signal regions are defined that select a minimum jet multiplicity and further require a large value of the ratio $E_{\mathrm{T}}^{\text {miss }} / \sqrt{H_{\mathrm{T}}}$, where $H_{\mathrm{T}}$ is the scalar sum of jet transverse momenta

$$
H_{\mathrm{T}}=\sum_{j} p_{\mathrm{T}, j}^{\mathrm{jet}}
$$

the sum being restricted to jets with $p_{\mathrm{T}}>40 \mathrm{GeV},|\eta|<2.8$. This ratio is approximately proportional to the significance of the $E_{\mathrm{T}}^{\text {miss }}$, under the assumption that the expected $E_{\mathrm{T}}^{\text {miss }}$ 
is zero and the resolution of the $E_{\mathrm{T}}^{\text {miss }}$ originates entirely from the stochastic variation in the jet momentum measurement. For jets with $p_{\mathrm{T}} \lesssim 1 \mathrm{TeV}$, the relative jet resolution scales approximately as $1 / \sqrt{p_{\mathrm{T}}}$.

Several auxiliary measurements are carried out in control and validation regions (VR) in order to define and constrain the major backgrounds to the analysis. Events selected at a lower jet multiplicity are used to extract the shape of the $E_{\mathrm{T}}^{\text {miss }} / \sqrt{H_{\mathrm{T}}}$ distribution, which is then extrapolated to the signal regions to quantify the multijet background, as described fully in section 6.1. The normalisation of the $t \bar{t}$ and $W+$ jets background components is adjusted to match data in control regions, using the procedure defined in section 6.2.

\subsection{Signal region definitions}

The common selection of events for all the signal regions is as follows. To limit the contribution of SM background processes in which neutrinos are produced, leading to significant $E_{\mathrm{T}}^{\text {miss }}$, events containing any preselected electron or muon following the overlap removal procedure are rejected.

Biases in the $E_{\mathrm{T}}^{\text {miss }}$ due to pile-up jets surviving the JVT selection are removed by excluding events for which a jet with $60<p_{\mathrm{T}}<70 \mathrm{GeV}$ and JVT $<0.59$ lies opposite to the $E_{\mathrm{T}}^{\text {miss }}\left(\Delta \phi\left(j_{i}, \vec{E}_{\mathrm{T}}^{\text {miss }}\right)>2.2\right)$. Likewise, events are rejected if they contain a jet with $p_{\mathrm{T}}>50 \mathrm{GeV}$ and $|\eta|<2.0$ pointing towards regions in which tile calorimeter modules were disabled. These requirements are also applied to the control regions and validation regions described later in section 5.2.1.

Subsequently, restrictions on the jet multiplicity $N_{\text {jet }}$ are imposed, depending on the analysis channel; only jets with $p_{\mathrm{T}}>50(80) \mathrm{GeV}$ and $|\eta|<2.0$ are considered as signal jets and therefore used in the $N_{\text {jet }}$ selection. These selections are abbreviated as $j 50$ (j80), for which the corresponding jet multiplicities are denoted $N_{\text {jet }}^{50}$ and $N_{\text {jet }}^{80}$. The lower and higher jet- $p_{\mathrm{T}}$ thresholds were optimised to permit sensitivity to a variety of potential SUSY mass spectra.

A threshold of $E_{\mathrm{T}}^{\text {miss }} / \sqrt{H_{\mathrm{T}}}>5 \mathrm{GeV}^{1 / 2}$ is the last element of the common selection. This criterion eliminates the vast majority of SM multijet and other background events with low $E_{\mathrm{T}}^{\text {miss }}$, while retaining sensitivity to a broad range of potential signals.

Next, the SRs in the two channels of the analysis are defined by a further categorisation of events.

\subsubsection{Heavy-flavour channel}

The following $N_{\text {jet }}$ values are considered in this channel: minimum $N_{\text {jet }}^{50} \in\{8,9,10,11\}$, and minimum $N_{\text {jet }}^{80} \in\{7,8,9\}$. Motivated by the desire to achieve good sensitivity to models with differing probabilities of heavy flavour jets being produced during cascade decays, three signal regions that respectively require $N_{b \text {-tag }} \geq 0,1,2$ are defined for each value of $N_{\text {jet }}$, where the $b$-jets must have $p_{\mathrm{T}}>50 \mathrm{GeV}$ and $|\eta|<2.0$.

\subsubsection{Jet mass channel}

Should sparticles be produced and decay through a long decay chain, or provide enough kinetic energy to significantly boost heavy particles such as top quarks and bosons, signal 


\begin{tabular}{|c|c|c|c|}
\hline Criterion & Heavy-flavo & r channel & Jet mass channel \\
\hline Jet $|\eta|$ & \multicolumn{3}{|c|}{$<2.0$} \\
\hline Jet $p_{\mathrm{T}}$ & $>50 \mathrm{GeV}$ & $>80 \mathrm{GeV}$ & $>50 \mathrm{GeV}$ \\
\hline$N_{\text {jet }}$ & $\geq 8,9,10,11$ & $\geq 7,8,9$ & $\geq 8,9,10$ \\
\hline Lepton veto & \multicolumn{3}{|c|}{ No preselected $e$ or $\mu$ after overlap removal } \\
\hline$b$-jet selection & \multirow{2}{*}{\multicolumn{3}{|c|}{$\begin{array}{c}p_{\mathrm{T}}>50 \mathrm{GeV} \text { and }|\eta|<2.0 \\
p_{\mathrm{T}}>100 \mathrm{GeV} \text { and }|\eta|<1.5\end{array}$}} \\
\hline Large-R-jet selection & & & \\
\hline$N_{b-\text { tag }}$ & \multicolumn{2}{|c|}{$\geq 0,1,2$} & $\geq 0$ \\
\hline$M_{\mathrm{J}}^{\Sigma}$ & \multicolumn{2}{|c|}{$\geq 0$} & $\geq 340,500 \mathrm{GeV}$ \\
\hline$E_{\mathrm{T}}^{\mathrm{miss}} / \sqrt{H_{\mathrm{T}}}$ & \multicolumn{3}{|c|}{$>5 \mathrm{GeV}^{1 / 2}$} \\
\hline
\end{tabular}

Table 1. Summary of the selection criteria for all signal regions used in this analysis. In each column, the three selection criteria on the number $N_{b \text {-tag }}$ of $b$-tagged jets or the two on the sum $M_{\mathrm{J}}^{\Sigma}$ of masses of large-radius jets are applied to define separate signal regions for each of the jet multiplicities considered.

events might be characterised not only by an unusually large jet multiplicity but also by the formation of large-radius jets with high masses. The kinematic structure of SM events, by contrast, does not produce a high rate of events containing large-radius jets with mass greater than the top quark mass.

For background discrimination in this channel, a selection variable, $M_{\mathrm{J}}^{\Sigma}$, is defined to be the sum of the masses $m_{j}^{R=1.0}$ of the large-radius jets

$$
M_{\mathrm{J}}^{\Sigma}=\sum_{j} m_{j}^{R=1.0}
$$

where the sum is over the large-radius jets that satisfy $p_{\mathrm{T}}^{R=1.0}>100 \mathrm{GeV}$ and $\left|\eta^{R=1.0}\right|<1.5$, as described in section 4. Two thresholds for $M_{\mathrm{J}}^{\Sigma}$ at $340 \mathrm{GeV}$ and $500 \mathrm{GeV}$, chosen following optimisation studies, define signal regions for $N_{\text {jet }}^{50} \in\{8,9,10\}$, while no $j 80$ SRs are defined. As these thresholds are approximately twice and thrice the top quark mass, the residual irreducible backgrounds are respectively from top quark pair production in association with vector bosons and four-top processes, both of which have a very small rate.

A summary of all signal region selections is given in table 1.

\subsection{Control region definitions}

For each signal region, three control regions are used to constrain the background predictions using data, and are split into two sets. The first set, referred to as the multijet template region (TR) selection, maintains the same lepton veto as used in the SR, but modifies the signal jet multiplicity or $E_{\mathrm{T}}^{\text {miss }} / \sqrt{H_{\mathrm{T}}}$ selection. Secondly, a pair of leptonic control regions are defined, classified according to the absence or presence of a $b$-tagged jet, in which the lepton veto is replaced with a requirement on the presence of exactly one signal electron or muon (henceforth referred to merely as "lepton", $\ell$ ). 


\subsubsection{Multijet template region}

Fundamental to this analysis is the extraction of an estimate of the multijet background directly from data, avoiding large theoretical uncertainties in the inclusive and differential cross-sections for these processes. The full estimation procedure is described in section 6.1.

Broadly, four different selections are used to evaluate the background prediction and its associated systematic uncertainties. The shape of the full $E_{\mathrm{T}}^{\text {miss }} / \sqrt{H_{\mathrm{T}}}$ distribution $\left(E_{\mathrm{T}}^{\text {miss }} / \sqrt{H_{\mathrm{T}}}\right.$ template) is measured in events containing exactly six signal jets with $p_{\mathrm{T}}>$ $50 \mathrm{GeV}$ for the $j 50$ signal regions and exactly five signal jets with $p_{\mathrm{T}}>80 \mathrm{GeV}$ for the $j 80$ signal regions. For normalisation of the template prediction, events are counted in a TR defined by the same signal jet multiplicity as the signal region, but an upper bound of $1.5 \mathrm{GeV}^{1 / 2}$ on the $E_{\mathrm{T}}^{\text {miss }} / \sqrt{H_{\mathrm{T}}}$ variable. Validation regions are defined that require seven signal jets with $p_{\mathrm{T}}>50 \mathrm{GeV}$ for the $j 50$ signal regions and six signal jets with $p_{\mathrm{T}}>80 \mathrm{GeV}$ for the $j 80$ signal regions, and also impose a minimum $E_{\mathrm{T}}^{\text {miss }} / \sqrt{H_{\mathrm{T}}}>5 \mathrm{GeV}^{1 / 2}$ threshold, as in the signal regions. Finally, an additional validation region is defined in the range $1.5<E_{\mathrm{T}}^{\text {miss }} / \sqrt{H_{\mathrm{T}}}<4.5 \mathrm{GeV}^{1 / 2}$, for each signal region jet multiplicity. The same $N_{b \text {-tag }}$ and $M_{\mathrm{J}}^{\Sigma}$ thresholds are applied in each template and validation region as in the corresponding signal region.

\subsubsection{Leptonic control regions}

Also important is the estimation of the next two largest background processes, $t \bar{t}$ and $W+$ jets, from MC simulation, as detailed in section 3.2.1. To correct for potential mismodelling of the process cross-sections and kinematics by the event generators, the normalisation for the background predictions is modified based on a simultaneous fit of the auxiliary measurements, explained in section 6.3.

The leptonic control regions constraining the $t \bar{t}$ and $W+$ jets normalisation are defined with identical selection criteria as their corresponding signal regions, apart from the following differences, summarised also in table 2:

1. Instead of rejecting events containing a preselected lepton, events must contain exactly one signal lepton with $p_{\mathrm{T}}>20 \mathrm{GeV}$.

2. To prevent contamination from potential signals, events must satisfy a requirement on the transverse mass $m_{\mathrm{T}}<120 \mathrm{GeV}$, where

$$
m_{\mathrm{T}}=\sqrt{2 p_{\mathrm{T}}^{\ell} E_{\mathrm{T}}^{\mathrm{miss}}\left[1-\cos \left(\Delta \phi\left(\vec{p}_{\mathrm{T}}^{\ell}, \vec{E}_{\mathrm{T}}^{\mathrm{miss}}\right)\right)\right]} .
$$

3. To increase the number of selected events, the minimum signal jet multiplicity $N_{\text {jet }}$ is reduced by one from the corresponding signal region. However, if the lepton satisfies the $p_{\mathrm{T}}$ and $\eta$ requirements imposed on signal jets, then it is treated as a signal jet for the purposes of this selection. This reflects the main mechanism by which $t \bar{t}$ and $W+$ jets events pass the signal region selection: misidentification of an electron or hadronically-decaying tau lepton as a jet, which can increase the jet multiplicity. Events with leptons which are unreconstructed as they lie outside of detector acceptance can also contribute to the signal regions, but are a subdominant contribution. 


\begin{tabular}{|ll|}
\hline Control regions & \\
\hline Lepton multiplicity & Exactly one signal $e$ or $\mu$ \\
Lepton $p_{\mathrm{T}}$ & $>20 \mathrm{GeV}$ \\
$m_{\mathrm{T}}$ & $<120 \mathrm{GeV}$ \\
Jet $p_{\mathrm{T}},|\eta|$ & Same as SR \\
Number of jets including lepton & $N_{\text {jet }}^{\mathrm{SR}}-1$ \\
$b$-jet multiplicity & $=0(W+$ jets $)$ or $\geq 1(t \bar{t})$ \\
$M_{\mathrm{J}}^{\Sigma}$ & Same as SR \\
$E_{\mathrm{T}}^{\mathrm{miss}} / \sqrt{H_{\mathrm{T}}}$ & $>3,4,5 \mathrm{GeV}^{1 / 2}$ \\
\hline
\end{tabular}

Table 2. Definition of the leptonic control regions, used to normalise the $t \bar{t}$ and $W+$ jets backgrounds. In the control regions, the lepton is recast as a jet if it satisfies the same kinematic criteria as the jets. Such leptons contribute to the $E_{\mathrm{T}}^{\text {miss }} / \sqrt{H_{\mathrm{T}}}$ (through $H_{\mathrm{T}}$ ) and also $M_{\mathrm{J}}^{\Sigma}$.

\begin{tabular}{|l|c|c|c|}
\hline Signal channel & \multicolumn{2}{|c|}{ Minimum SR $N_{\text {jet }}$} & $E_{\mathrm{T}}^{\text {miss }} / \sqrt{H_{\mathrm{T}}}$ threshold \\
\hline \multirow{4}{*}{ Heavy-flavour channel } & Jet $p_{\mathrm{T}}>50 \mathrm{GeV}$ & Jet $p_{\mathrm{T}}>80 \mathrm{GeV}$ & \\
& 8,9 & 7 & $>5 \mathrm{GeV}^{1 / 2}$ \\
& 10 & 8 & $>4 \mathrm{GeV}^{1 / 2}$ \\
& 11 & 9 & $>3 \mathrm{GeV}^{1 / 2}$ \\
\hline \multirow{5}{*}{ Jet mass channel } & $M_{\mathrm{J}}^{\Sigma}>340 \mathrm{GeV}$ & $M_{\mathrm{J}}^{\Sigma}>500 \mathrm{GeV}$ & \\
& 8 & - & $>5 \mathrm{GeV}^{1 / 2}$ \\
& 9 & 8 & $>3 \mathrm{GeV}^{1 / 2}$ \\
& 10 & 9,10 & $\mathrm{GeV}^{1 / 2}$ \\
\hline
\end{tabular}

Table 3. The $E_{\mathrm{T}}^{\text {miss }} / \sqrt{H_{\mathrm{T}}}$ thresholds for the control regions corresponding to each signal region. In each case, the same $E_{\mathrm{T}}^{\text {miss }} / \sqrt{H_{\mathrm{T}}}$ threshold is used for both the $W+$ jets and $t \bar{t}$ control regions.

4. Events consistent with $W+$ jets and $t \bar{t}$ production are separated by means of the $N_{b \text {-tag }}$ selection; the $W+$ jets $\mathrm{CR}$ requires $N_{b \text {-tag }}=0$ while the $t \bar{t}$ CR requires $N_{b \text {-tag }} \geq 1$.

5. The $E_{\mathrm{T}}^{\text {miss }} / \sqrt{H_{\mathrm{T}}}$ threshold is lowered from $5 \mathrm{GeV}^{1 / 2}$ to $3 \mathrm{GeV}^{1 / 2}$ or $4 \mathrm{GeV}^{1 / 2}$ when it is necessary to increase the statistical precision of the measurement. The $E_{\mathrm{T}}^{\text {miss }} / \sqrt{H_{\mathrm{T}}}$ thresholds are specified in table 3 .

\section{Background estimation techniques}

\subsection{Multijet template estimation}

Accurate modelling of multijet processes by performing QCD calculations involving high multiplicity multi-leg matrix elements is difficult. This is compounded by the challenges of reproducing events populating the tails of the detector response, representative of the high- $E_{\mathrm{T}}^{\text {miss }}$ events selected in this analysis. Hence, to confidently estimate the multijet background component, which makes up 50-70\% of the total SM expectation, the prediction is based on direct measurements in data. 
The strategy used in this analysis is based on the observation that the $E_{\mathrm{T}}^{\text {miss }} / \sqrt{H_{\mathrm{T}}}$ spectrum for selected multijet events is primarily determined by the calorimeter response to jets, which is approximately independent of how the total jet transverse energy $H_{\mathrm{T}}$ is partitioned between the jets. Thus, the $E_{\mathrm{T}}^{\text {miss }} / \sqrt{H_{\mathrm{T}}}$ spectrum measured in events with a lower jet multiplicity does not greatly differ from that observed in events with a high jet multiplicity. A template for the multijet $E_{\mathrm{T}}^{\text {miss }} / \sqrt{H_{\mathrm{T}}}$ distribution can thus be extracted in a selection complementary to the signal region, specifically the template regions defined in section 5.2.1. At larger values of $E_{\mathrm{T}}^{\text {miss }} / \sqrt{H_{\mathrm{T}}}$, it is necessary to subtract from the data the expected contributions due to SM processes producing neutrinos. For the $E_{\mathrm{T}}^{\text {miss }} / \sqrt{H_{\mathrm{T}}}$ threshold used in the SRs, such contributions comprise approximately $10 \%$ to $50 \%$ of the total. These predictions are determined from MC simulation. This template also accounts for smaller background contributions from $t \bar{t}$ production with fully hadronic decays as well as $\gamma+$ jets.

By the logic above, the multijet prediction $n_{\text {multijet }}$ for the number of events with $b<E_{\mathrm{T}}^{\text {miss }} / \sqrt{H_{\mathrm{T}}}<c$ for a SR based on a TR can be written as follows:

$$
\begin{aligned}
n_{\text {multijet }}^{\mathrm{SR}, b<E_{\mathrm{T}}^{\mathrm{miss}} / \sqrt{H_{\mathrm{T}}}<c} & =\frac{n_{\text {multijet }}^{\mathrm{SR}, E_{\mathrm{T}}^{\text {miss }} / \sqrt{H_{\mathrm{T}}}<a}}{n_{\text {multijet }}^{\mathrm{TR}, E_{\mathrm{T}}^{\mathrm{miss}} / \sqrt{H_{\mathrm{T}}}<a}} \cdot n_{\text {multijet }}^{\mathrm{TR}, b<E_{\mathrm{T}}^{\mathrm{miss}} / \sqrt{H_{\mathrm{T}}}<c} \\
& =\frac{n_{\text {multijet }}^{\mathrm{SR}, E_{\mathrm{T}}^{\text {miss }} / \sqrt{H_{\mathrm{T}}}<a}}{n_{\text {multijet }}^{\mathrm{TR}, E_{\mathrm{T}}^{\mathrm{miss}} / \sqrt{H_{\mathrm{T}}}<a}} \cdot\left(n_{\text {obs }}^{\mathrm{TR}, b<E_{\mathrm{T}}^{\mathrm{miss}} / \sqrt{H_{\mathrm{T}}}<c}-n_{\mathrm{MC} \nu}^{\mathrm{TR}, b E_{\mathrm{T}}^{\mathrm{miss}} / \sqrt{H_{\mathrm{T}}}<c}\right) .
\end{aligned}
$$

The normalisation of the template is fixed in the range $E_{\mathrm{T}}^{\text {miss }} / \sqrt{H_{\mathrm{T}}}<a$ such that $a<b<c$, which is entirely dominated by multijet events. In the template region, the observation in data is denoted $n_{\mathrm{obs}}$, while the predicted number of events with neutrinos is written $n_{\mathrm{MC} \nu}$.

While the exact division of $H_{\mathrm{T}}$ among the multiple jets in a single event does not significantly influence the template independence, the distribution of $H_{\mathrm{T}}$ itself is forced higher as the $N_{\text {jet }}$ requirements are made more stringent. This implies the existence of an indirect correlation between the $E_{\mathrm{T}}^{\mathrm{miss}} / \sqrt{H_{\mathrm{T}}}$ and the jet multiplicity, which challenges the earlier assumption of template independence. It is therefore necessary to extract the multijet template in several bins of $H_{\mathrm{T}}$ in order to remove the subdominant residual dependence of $E_{\mathrm{T}}^{\mathrm{miss}} / \sqrt{H_{\mathrm{T}}}$ on $H_{\mathrm{T}}$. The lower bin boundaries are set at $0,600,900$ and $1200 \mathrm{GeV}$, which was found to be sufficient to remove the dependence of the template on $H_{\mathrm{T}}$. Predictions for each bin are derived independently and summed to obtain the total SR expectation.

The dependence of the template prediction on pile-up was studied in detail. While the width of the $E_{\mathrm{T}}^{\text {miss }} / \sqrt{H_{\mathrm{T}}}$ distribution itself shows a correlation with the amount of pile-up, both due to the growth of the jet resolution and the influence of additional jets present in the event, increases in the amount of pile-up do not worsen the closure of the template prediction, i.e. the ability of the method to correctly predict the multijet background in validation regions free of signal. This demonstrates that the template method accurately captures the variation in the $E_{\mathrm{T}}^{\text {miss }} / \sqrt{H_{\mathrm{T}}}$ spectrum under changing LHC conditions, and that there is no observable bias in the signal region acceptance for a given level of pile-up. 
Other potential influences on the closure of the template such as the heavy-flavour composition were also studied carefully. The most important of these effects are kinematic variations between the template and signal regions, and differences in the number of heavy-flavour jets in the two event selections. While no major changes in the prediction were observed in these checks, systematic uncertainties that estimate the sensitivity of the template prediction to these variables are assessed.

Kinematic differences are covered by comparing the nominal estimate to the prediction extracted using an alternative $H_{\mathrm{T}}$-binning strategy, setting the bin boundaries instead at $0,600,800,1000,1200$ and $1400 \mathrm{GeV}$. The resulting uncertainty is typically $5-10 \%$ in the SRs.

An uncertainty due to jet flavour composition is determined as the difference between the nominal estimate, which assumes an identical flavour composition between the TR and SRs, and a $\chi^{2}$ fit that interpolates between the nominal estimate and a flavour-split template estimate. The flavour-split template prediction is produced by separating the template and signal regions into two bins, one requiring exactly as many $b$-jets as in the SR $N_{b \text {-tag }}$ selection, and the other requiring at least one more. A $\chi^{2}$ fit to data in the validation regions is then used to linearly combine the nominal and flavour-split templates. The resulting combined template is used as a basis for comparison to the nominal prediction. This procedure ensures that an appropriate uncertainty is estimated if the nominal estimate is significantly different from the best fit; if the naïve flavour-split estimate describes the data poorly, this does not result in an overestimate of the uncertainty. For the jet mass channel, this uncertainty ranges from $3 \%$ to $6 \%$. It is larger in the heavy-flavour channel: at most $20 \%$ in the tightest selections, and up to $12 \%$ elsewhere.

Finally, to account for other potential sources of mismodelling, an overall closure uncertainty is computed. This is defined as the maximal relative difference between the template prediction and the observation in data for the VRs defined in section 5.2.1, either with a lower jet multiplicity or a reduced $E_{\mathrm{T}}^{\text {miss }} / \sqrt{H_{\mathrm{T}}}$ value. The template closure is checked in a VR at a lower jet multiplicity but with the same $E_{\mathrm{T}}^{\text {miss }} / \sqrt{H_{\mathrm{T}}}>5 \mathrm{GeV}^{1 / 2}$ threshold as in the $\mathrm{SR}$, or in several bins of $E_{\mathrm{T}}^{\mathrm{miss}} / \sqrt{H_{\mathrm{T}}}$ :

$$
E_{\mathrm{T}}^{\mathrm{miss}} / \sqrt{H_{\mathrm{T}}} \in(1.5,2.0),(2.0,3.0),(3.0,4.0) \mathrm{GeV}^{1 / 2} .
$$

Example distributions of $E_{\mathrm{T}}^{\text {miss }} / \sqrt{H_{\mathrm{T}}}$ in the lower-jet-multiplicity VRs are shown in figure 2 . The degree of closure varies, generally ranging between $8 \%$ and $12 \%$ and extending to $30 \%$ for regions with the fewest events.

\subsection{Leptonic background estimates}

All background contributions from processes in which $W \rightarrow \ell \nu$ or $Z \rightarrow \nu \nu$ decays produce neutrinos, including single or pair production of top quarks and electroweak vector bosons, are estimated using MC simulation. The two largest of these, $t \bar{t}$ and $W+$ jets, are responsible for $20-45 \%$ and up to $10 \%$ of the SM background respectively. Other processes, such as $Z+$ jets, single top and diboson production collectively make up no more than $12 \%$ of the total SR expectation. As such, corrections to the size of the $t \bar{t}$ and $W+$ jets background 


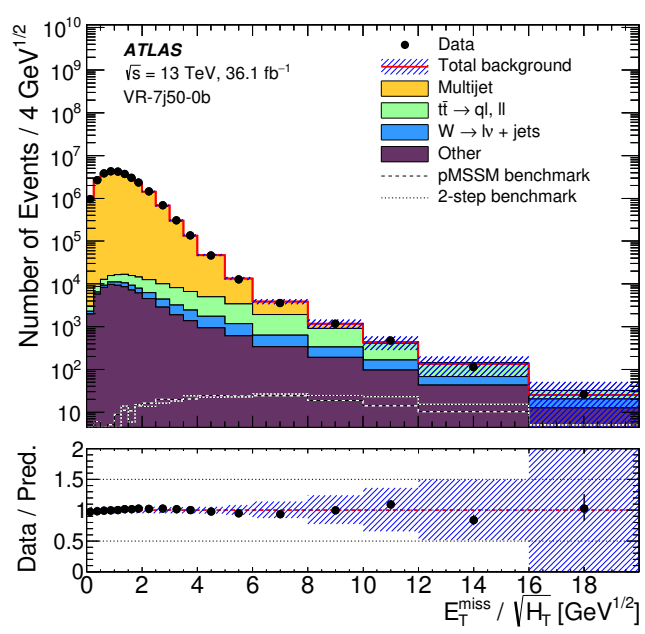

(a) $N_{\text {jet }}^{50}=7, N_{b \text {-tag }} \geq 0$

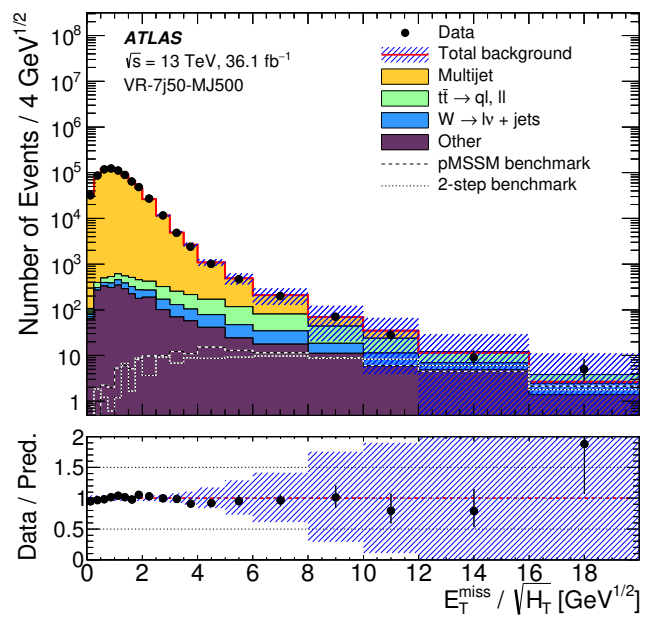

(b) $N_{\text {jet }}^{50}=7, M_{\mathrm{J}}^{\Sigma}>500 \mathrm{GeV}$

Figure 2. Distributions of the $E_{\mathrm{T}}^{\text {miss }} / \sqrt{H_{\mathrm{T}}}$ for events in the validation regions for the $50 \mathrm{GeV}$ jet $p_{\mathrm{T}}$ threshold in the heavy-flavour channel (a) and a $M_{\mathrm{J}}^{\Sigma}$ selection (b). The blue hatched band indicates the quadrature sum of the statistical uncertainty from MC simulated samples and the various systematic uncertainties in the background prediction. The dashed lines labelled 'pMSSM' and '2-step' refer to benchmark signal points - a pMSSM slice model with $\left(m_{\tilde{g}}, m_{\tilde{\chi}_{1}^{ \pm}}\right)=(1400,200) \mathrm{GeV}$ and a cascade decay model with $\left(m_{\tilde{g}}, m_{\tilde{\chi}_{1}^{0}}\right)=(1400,200) \mathrm{GeV}$. The lower panels show the ratio of the observed data to the total SM background.

components, together with the multijet template estimate previously described, provide a sufficiently accurate background prediction for this search. For each of the $t \bar{t}, W+$ jets and multijets background processes, a normalisation factor $\mu$ is determined, based on a likelihood fit described in section 6.3.

Control regions defined as in section 5.2.2 provide enriched samples of events from the relevant processes, in a kinematic region close to the signal selection. The purity of the CRs is around $85 \%$ for $t \bar{t}$ and typically $25-50 \%$ for $W+$ jets. As only these two processes contribute substantially to the CR populations, this level of purity is adequate to constrain the normalisations for both well.

Distributions of the number of jets $\left(p_{\mathrm{T}}>20 \mathrm{GeV},|\eta|<2.8\right)$ are shown in figure 3 for a selection of the $t \bar{t}$ and $W+$ jets CRs.

\subsection{Combined background fits}

For each background process constrained by the fit, an unconstrained normalisation factor $\mu_{b}, b \in\{t \bar{t}, W$, multijet $\}$ is defined, such that $\mu_{b}=1$ implies consistency with the nominal

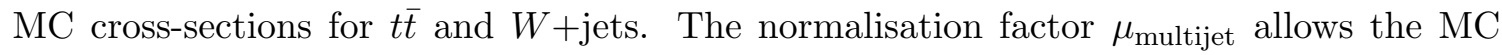
subtraction applied in the template estimate to be corrected by the CR measurements, and to be modified coherently with any systematic variations applied to the MC simulation.

A likelihood is then constructed for the ensemble of measurements in the control regions as the product of Poisson distributions whose means are specified by the nominal MC 


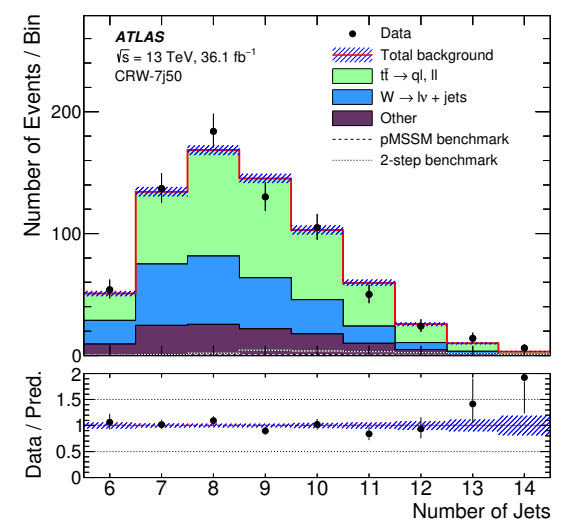

(a) $N_{\text {jet }}^{50} \geq 7, N_{b \text {-tag }}=0$

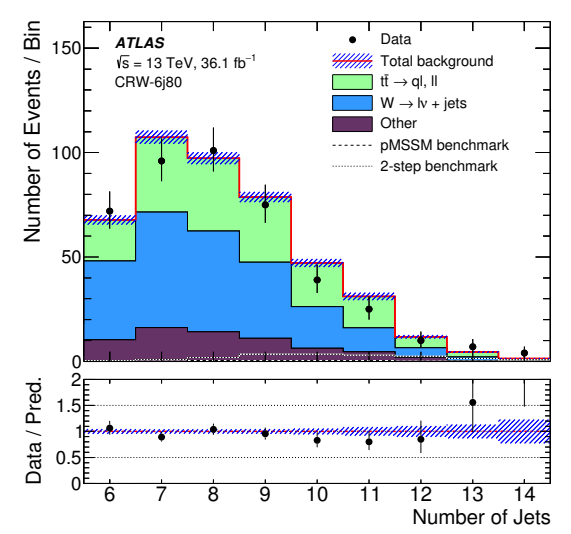

(c) $N_{\text {jet }}^{80} \geq 6, N_{b-\text { tag }}=0$

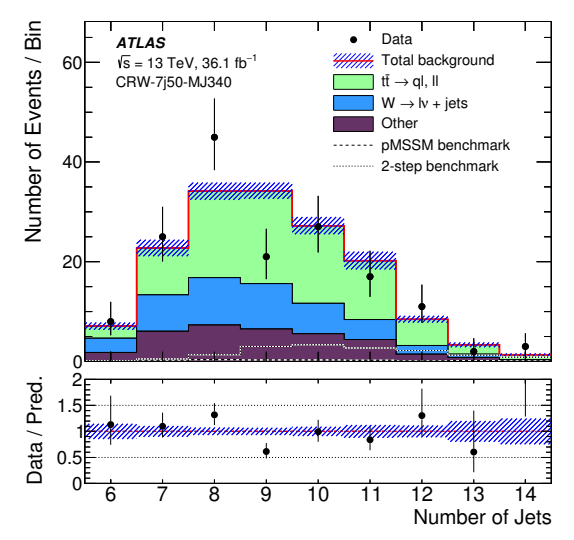

(e) $N_{\text {jet }}^{50} \geq 7, M_{\mathrm{J}}^{\Sigma}>340 \mathrm{GeV}, N_{b \text {-tag }}=0$

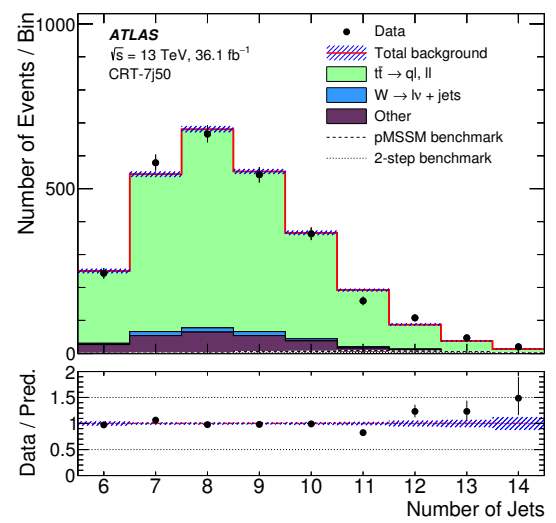

(b) $N_{\text {jet }}^{50} \geq 7, N_{b-\operatorname{tag}} \geq 1$

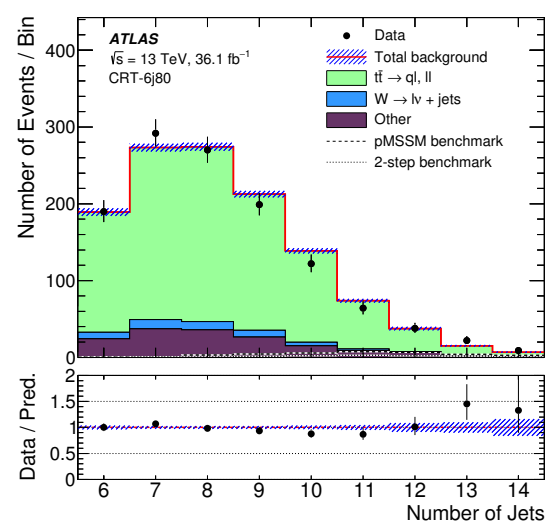

(d) $N_{\text {jet }}^{80} \geq 6, N_{b-\operatorname{tag}} \geq 1$

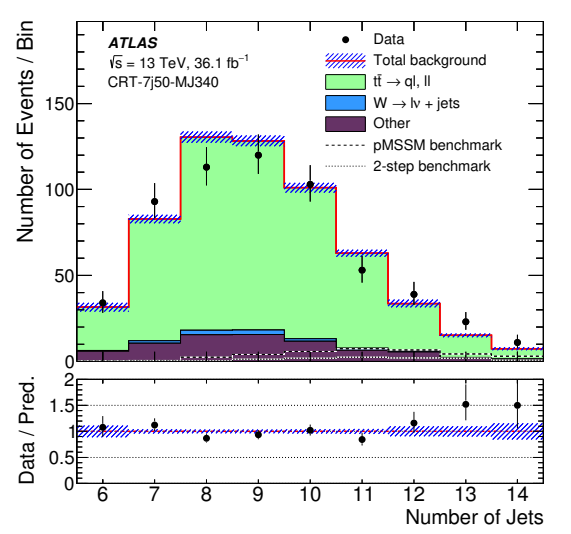

(f) $N_{\text {jet }}^{50} \geq 7, M_{\mathrm{J}}^{\Sigma}>340 \mathrm{GeV}, N_{b \text {-tag }} \geq 1$

Figure 3. The distribution of the number of jets observed in the $W+$ jets (left) and $t \bar{t}$ (right) control regions with the lowest jet multiplicities. The backgrounds are scaled by the normalisation factors extracted from the fit, described in section 6.3. The blue hatched band indicates the statistical uncertainty from MC simulated samples. The dashed lines labelled 'pMSSM' and '2-step' refer to benchmark signal points - a pMSSM slice model with $\left(m_{\tilde{g}}, m_{\tilde{\chi}_{1}^{ \pm}}\right)=(1400,200) \mathrm{GeV}$ and a cascade decay model with $\left(m_{\tilde{g}}, m_{\tilde{\chi}_{1}^{0}}\right)=(1400,200) \mathrm{GeV}$. The lower panels show the ratio of the observed data to the total SM background. 
estimate for that region, including the free normalisation factors $\mu_{b}$ [102]. For $\mu_{t \bar{t}}$ and $\mu_{W}$, the corresponding leptonic control regions provide the constraints. The 6 -jet $(j 50)$ or 5 -jet (j80) template region is treated as another control region in the fit such that $\mu_{\text {multijet }}$ is coupled to any modifications of $\mu_{t \bar{t}}$ and $\mu_{W}$. If $\mu_{t \bar{t}}=\mu_{W}=1$, then $\mu_{\text {multijet }}=1$ by construction, as the same region is used to derive the nominal multijet estimate. The systematic uncertainties (see section 7.1) are implemented in the form of Gaussian-constrained nuisance parameters modifying the Poisson mean of each background component contributing to the estimate in a given signal or control region.

Minimisation of the likelihood (profiling) fixes the values of, and uncertainties in, $\mu_{b}$, which can then be combined with the $\mathrm{MC}$ and template predictions to obtain the total background prediction in the signal region. The compatibility of the background prediction and SR observation is computed in the form of a $p$-value $\mathrm{CL}_{\mathrm{b}}$ which is the probability of an upwards fluctuation from the SR prediction no larger than that observed in data, given the background model.

\section{Statistical procedures}

\subsection{Systematic uncertainties}

Systematic uncertainties affecting this analysis are grouped into the three following sources.

Uncertainties from experimental sources include those in identification and reconstruction efficiencies, as well as energy and momentum scales and resolutions. They are assessed for all simulated event samples. Efficiency uncertainties are considered for hard-scatter jet selection, flavour tagging and selection of electrons and muons. Of these, only flavourtagging uncertainties have a non-negligible effect on the total background expectation in the signal regions; at most $4 \%$ in the heavy-flavour-enriched SRs.

The energy/momentum uncertainties affect jets, electrons, muons and photons, and are also propagated to the missing transverse momentum. Jet energy scale and resolution systematic uncertainties contribute $6-12 \%$ to the uncertainty in the SR yields. The soft term of the $E_{\mathrm{T}}^{\mathrm{miss}}$ also has its own associated uncertainties, which in the jet mass channel may have up to an $8 \%$ effect. In this category also fall the uncertainty in the total integrated luminosity considered for analysis as well as in the total inelastic $p p$ cross-section, which affects the simulation of pile-up $(<1 \%)$.

Theoretical uncertainties in the event generation affect both the background and signal MC samples. These are assessed by varying the matrix element and parton shower generators used, or by modifying scales (renormalisation, factorisation, resummation, matching) involved in the process calculations. Variation in the degree of additional QCD radiation accompanying $t \bar{t}$ production is the single largest source of uncertainty in the SRs (10-25\%); parton shower uncertainties play a subdominant role, typically being half as large as, but occasionally comparable to, the radiation systematic uncertainty. Constant uncertainties of $30 \%$ and $50 \%$ respectively are applied to the normalisation of diboson production and top quark pair production in association with vector bosons, and have an overall negligible effect on the analysis results. 
As described in section 6.1, uncertainties in the multijet background estimates are assessed, where kinematic and flavour differences between the template and signal regions are considered. An additional overall systematic uncertainty is ascribed for general non-closure of the template prediction. Apart from in the jet mass channel SRs, where the kinematic and flavour uncertainties are at most 3\%, and in the most statistically limited SRs, the uncertainties from the three sources are similar in magnitude. Where the statistical precision is poorer, fluctuations can drive the non-closure uncertainty up to $18 \%$.

\subsection{Hypothesis testing}

For the interpretation of the signal region observations, the likelihood fits for background estimation (section 6.3) are extended to perform two forms of hypothesis tests using a profile-likelihood-ratio test statistic [103], quantifying the significance of any observed excesses or the lack thereof. The discovery test discriminates between the null hypothesis stating that the SR measurement is consistent with only SM contributions and an alternative hypothesis postulating a positive signal. Conversely, any given signal model can be examined in an exclusion test of the signal-plus-background hypothesis, where an observation significantly smaller than the combination of SM and SUSY processes would lead to rejection of the signal model.

Taking into account all background predictions, normalisation factors and systematic uncertainties, the fit is implemented by including the SR in the ensemble of measurements and adding an additional signal component solely in the SR. Using a profile-likelihood-ratio test, the discovery $p$-value $p_{0}$, corresponding to the probability of an upward fluctuation in the absence of any signal, can be determined. This configuration also permits an upper limit on the visible signal cross-section to be set for an arbitrary signal, where it is assumed that the signal contamination in the control regions is negligible.

Exclusion testing of a chosen signal model proceeds similarly, but a signal component is allowed in all control regions as well as the signal region, to correct for potential signal contamination (which has been verified to be small). Theoretical and experimental systematic uncertainties in the signal MC simulation are included in the fit. A profile-likelihood-ratio test is then made of the compatibility between the best-fit $\mu_{\text {signal }}$ from data and the nominal signal hypothesis, corresponding to a signal strength $\mu_{\text {signal }}=1$. This provides the exclusion $p$-value $p_{1}$. Points in the SUSY parameter space are considered excluded if the $\mathrm{CL}_{\mathrm{s}}$ parameter, computed as $p_{1} /\left(1-\mathrm{CL}_{\mathrm{b}}\right)$, is smaller than 0.05 [104]. This protects against spurious exclusion of signals due to observing SR event counts significantly smaller than those predicted. While not strictly defining a frequentist confidence level, these are referred to as $95 \%$ confidence level CL limits.

\section{Results and interpretation}

The expected and observed event counts in the leptonic control regions are evaluated and normalisation factors derived. In general, the $t \bar{t}$ normalisation is close to one for lower jet multiplicities but may be as small as 0.71 for high jet multiplicities. For $\mu_{W}$, the range is typically $0.3-0.6$. Correspondingly, $\mu_{\text {multijet }}$ is corrected upwards by up to $24 \%$. 


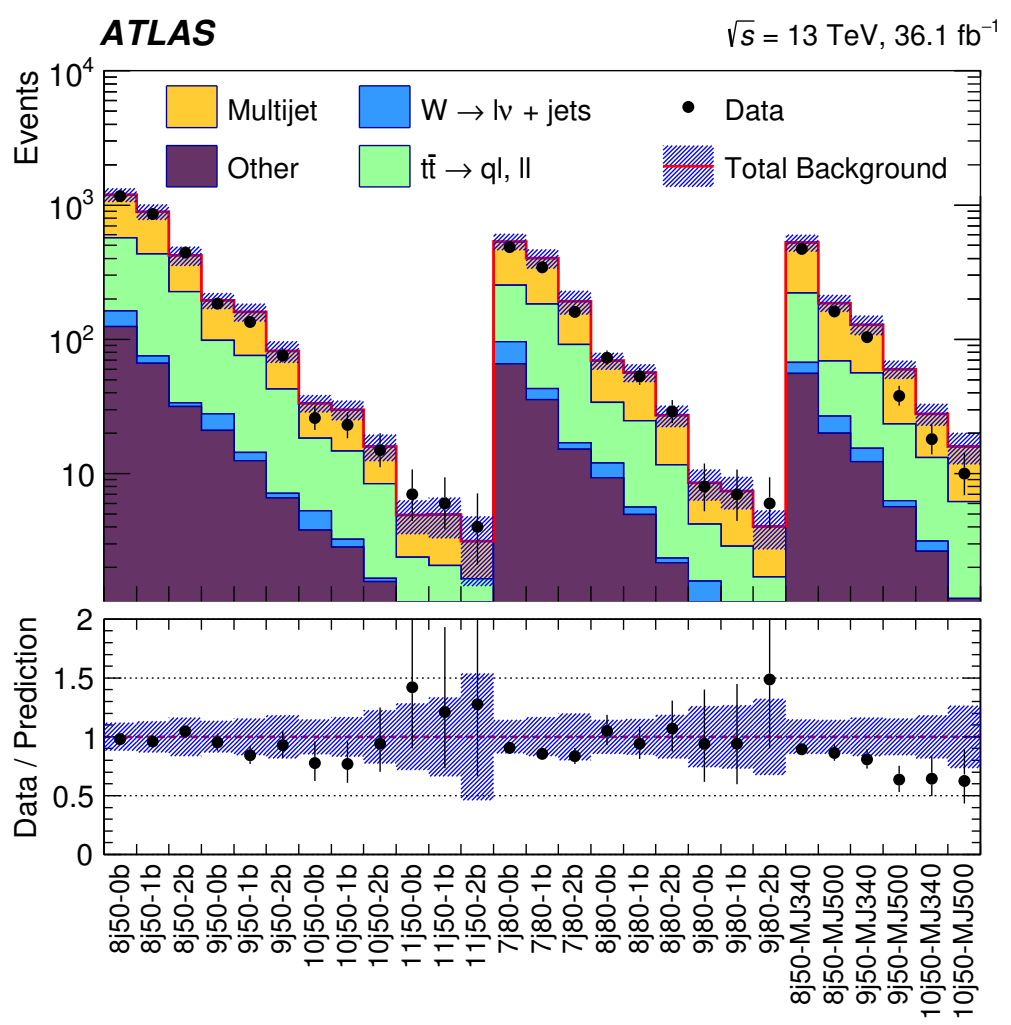

Figure 4. Summary plot showing the data and SM predictions constrained by the likelihood fit for all signal regions. Systematic and statistical uncertainties are shown in the blue hatched band, accounting for (anti-)correlations in their effects on different background components. The lower panels show the ratio of the observed data to the total SM background.

Signal region yields as observed in data are summarised in table 4. These are illustrated graphically in figure 4 . The most significant difference from the SM prediction is a deficit in the 9j MJ500 region with a statistical significance of $1.8 \sigma$ and a corresponding $p$-value $\left(1-\mathrm{CL}_{\mathrm{b}}\right)$ of 0.04 . Similar deficits are observed in the other MJ SRs, but the large overlap between these SRs implies that the deficits are strongly correlated.

The full distributions of $E_{\mathrm{T}}^{\text {miss }} / \sqrt{H_{\mathrm{T}}}$ are shown for two of the most sensitive signal regions in figure 5. For all signal regions, the data agree with the predicted $E_{\mathrm{T}}^{\text {miss }} / \sqrt{H_{\mathrm{T}}}$ distributions within the systematic uncertainties.

Table 5 quantifies the results of the fit to all signal regions. When testing for a positive signal, the smallest $p_{0}$ value observed is 0.2 , for $N_{\text {jet }}^{80} \geq 9$ and $N_{b \text {-tag }} \geq 2$. The strongest limits set on the visible cross-section are of about $0.19 \mathrm{fb}$, for $N_{\text {jet }}^{50} \geq 11$ and $N_{b \text {-tag }} \geq 2$.

\subsection{Exclusion limits}

Using the exclusion configuration defined in section 7.2, limits are set at the 95\% CL in the signal scenarios described in section 3.2.2. Constraints from all 27 SRs are combined by considering only the SR with the best expected exclusion sensitivity at each signal model point. These are illustrated in several parameter planes in figures 6 and 7 . 


\begin{tabular}{|c|c|c|c|c|c|}
\hline \multirow{2}{*}{\multicolumn{2}{|c|}{ Signal region }} & \multicolumn{3}{|c|}{ Fitted background } & \multirow{2}{*}{ Obs event } \\
\hline & & Multijet & Leptonic & Total & \\
\hline \multirow{3}{*}{$N_{\text {jet }}^{50} \geq 8$} & $N_{b-\text { jet }} \geq 0$ & $622 \pm 42$ & $570 \pm 140$ & $1190 \pm 140$ & 1169 \\
\hline & $N_{b-\text { jet }} \geq 1$ & $460 \pm 50$ & $430 \pm 110$ & $890 \pm 140$ & 856 \\
\hline & $N_{b \text {-jet }} \geq 2$ & $196 \pm 39$ & $226 \pm 57$ & $422 \pm 81$ & 442 \\
\hline \multirow{3}{*}{$N_{\text {jet }}^{50} \geq 9$} & $N_{b-\text { jet }} \geq 0$ & $96 \pm 11$ & $98 \pm 24$ & $194 \pm 28$ & 185 \\
\hline & $N_{b \text {-jet }} \geq 1$ & $84 \pm 15$ & $76 \pm 20$ & $160 \pm 31$ & 135 \\
\hline & $N_{b-\text { jet }} \geq 2$ & $39 \pm 12$ & $42.5 \pm 9.5$ & $82 \pm 19$ & 76 \\
\hline \multirow{3}{*}{$N_{\text {jet }}^{50} \geq 10$} & $N_{b-\text { jet }} \geq 0$ & $15.1 \pm 3.0$ & $18.3 \pm 3.9$ & $33.5 \pm 5.1$ & 26 \\
\hline & $N_{b \text {-jet }} \geq 1$ & $15.3 \pm 3.7$ & $14.7 \pm 3.3$ & $30.0 \pm 5.9$ & 23 \\
\hline & $N_{b \text {-jet }} \geq 2$ & $7.6 \pm 3.1$ & $8.4 \pm 1.8$ & $16.0 \pm 4.2$ & 15 \\
\hline \multirow{3}{*}{$N_{\text {jet }}^{50} \geq 11$} & $N_{b-\text { jet }} \geq 0$ & $2.54 \pm 0.76$ & $2.4 \pm 1.2$ & $4.9 \pm 1.2$ & 7 \\
\hline & $N_{b-\text { jet }} \geq 1$ & $2.88 \pm 0.84$ & $2.1 \pm 1.4$ & $5.0 \pm 1.3$ & 6 \\
\hline & $N_{b \text {-jet }} \geq 2$ & $1.49 \pm 0.72$ & $1.6 \pm 1.5$ & $3.1 \pm 1.5$ & 4 \\
\hline \multirow{3}{*}{$N_{\text {jet }}^{80} \geq 7$} & $N_{b-\text { jet }} \geq 0$ & $282 \pm 32$ & $253 \pm 69$ & $535 \pm 74$ & 486 \\
\hline & $N_{b \text {-jet }} \geq 1$ & $219 \pm 28$ & $183 \pm 60$ & $402 \pm 74$ & 343 \\
\hline & $N_{b \text {-jet }} \geq 2$ & $100 \pm 17$ & $91 \pm 34$ & $191 \pm 44$ & 160 \\
\hline \multirow{3}{*}{$N_{\text {jet }}^{80} \geq 8$} & $N_{b \text {-jet }} \geq 0$ & $35.7 \pm 5.6$ & $33.8 \pm 8.3$ & $70 \pm 10$ & 73 \\
\hline & $N_{b-\text { jet }} \geq 1$ & $31.6 \pm 5.7$ & $24.8 \pm 6.4$ & $56 \pm 10$ & 53 \\
\hline & $N_{b \text {-jet }} \geq 2$ & $15.5 \pm 3.8$ & $11.6 \pm 3.3$ & $27.1 \pm 6.0$ & 29 \\
\hline \multirow{3}{*}{$N_{\text {jet }}^{80} \geq 9$} & $N_{b-\text { jet }} \geq 0$ & $4.3 \pm 1.3$ & $4.2 \pm 1.8$ & $8.5 \pm 2.0$ & 8 \\
\hline & $N_{b-\text { jet }} \geq 1$ & $4.5 \pm 1.3$ & $2.9 \pm 1.5$ & $7.4 \pm 1.8$ & 7 \\
\hline & $N_{b-\text { jet }} \geq 2$ & $2.34 \pm 0.95$ & $1.69 \pm 0.89$ & $4.0 \pm 1.2$ & 6 \\
\hline \multirow{2}{*}{$N_{\text {jet }}^{50} \geq 8$} & $M_{\mathrm{J}}^{\Sigma} \geq 340 \mathrm{GeV}$ & $306 \pm 54$ & $220 \pm 55$ & $526 \pm 72$ & 471 \\
\hline & $M_{\mathrm{J}}^{\Sigma} \geq 500 \mathrm{GeV}$ & $118 \pm 18$ & $69 \pm 20$ & $187 \pm 24$ & 161 \\
\hline \multirow{2}{*}{$N_{\mathrm{jet}}^{50} \geq 9$} & $M_{\mathrm{J}}^{\Sigma} \geq 340 \mathrm{GeV}$ & $73 \pm 15$ & $56 \pm 15$ & $129 \pm 23$ & 104 \\
\hline & $M_{\mathrm{J}}^{\Sigma} \geq 500 \mathrm{GeV}$ & $36.5 \pm 6.3$ & $23.3 \pm 7.0$ & $60 \pm 10$ & 38 \\
\hline \multirow{2}{*}{$N_{\text {jet }}^{50} \geq 10$} & $M_{\mathrm{J}}^{\Sigma} \geq 340 \mathrm{GeV}$ & $14.6 \pm 3.8$ & $13.2 \pm 3.5$ & $27.9 \pm 5.7$ & 18 \\
\hline & $M_{\mathrm{J}}^{\Sigma} \geq 500 \mathrm{GeV}$ & $9.8 \pm 2.6$ & $6.2 \pm 3.3$ & $16.0 \pm 4.7$ & 10 \\
\hline
\end{tabular}

Table 4. The expected SM background (and separately the multijet and leptonic contributions) and the observed number of data events. The SM background normalisations are obtained from fits to the data in control regions, as described in the text. 


\begin{tabular}{|c|c|c|c|c|c|c|}
\hline \multicolumn{2}{|c|}{ Signal Region } & $\langle\epsilon \sigma\rangle_{\text {obs }}^{95}[\mathrm{fb}]$ & $S_{\text {obs }}^{95}$ & $S_{\exp }^{95}$ & $1-\mathrm{CL}_{\mathrm{b}}$ & $p_{0}$ \\
\hline \multirow{3}{*}{$N_{\text {jet }}^{50} \geq 8$} & $N_{b \text {-jet }} \geq 0$ & 7.2 & 260 & $270_{-70}^{+90}$ & 0.44 & 0.50 \\
\hline & $N_{b \text {-jet }} \geq 1$ & 6.4 & 230 & $250_{-60}^{+80}$ & 0.40 & 0.50 \\
\hline & $N_{b-\text { jet }} \geq 2$ & 4.6 & 170 & $160_{-40}^{+50}$ & 0.59 & 0.40 \\
\hline \multirow{3}{*}{$N_{\text {jet }}^{50} \geq 9$} & $N_{b-\text { jet }} \geq 0$ & 1.5 & 53 & $58_{-15}^{+20}$ & 0.38 & 0.50 \\
\hline & $N_{b-\text { jet }} \geq 1$ & 1.2 & 44 & $55_{-14}^{+18}$ & 0.24 & 0.50 \\
\hline & $N_{b-\text { jet }} \geq 2$ & 1.0 & 35 & $38_{-9}^{+12}$ & 0.40 & 0.50 \\
\hline \multirow{3}{*}{$N_{\text {jet }}^{50} \geq 10$} & $N_{b-\text { jet }} \geq 0$ & 0.30 & 11 & $15_{-4}^{+6}$ & 0.17 & 0.50 \\
\hline & $N_{b \text {-jet }} \geq 1$ & 0.31 & 11 & $15_{-4}^{+6}$ & 0.20 & 0.50 \\
\hline & $N_{b \text {-jet }} \geq 2$ & 0.31 & 11 & $12_{-3}^{+5}$ & 0.44 & 0.50 \\
\hline \multirow{3}{*}{$N_{\mathrm{jet}}^{50} \geq 11$} & $N_{b-\text { jet }} \geq 0$ & 0.23 & 8.5 & $6.3_{-1.5}^{+3.0}$ & 0.80 & 0.21 \\
\hline & $N_{b-\text { jet }} \geq 1$ & 0.21 & 7.4 & $6.5_{-1.7}^{+2.6}$ & 0.68 & 0.34 \\
\hline & $N_{b-\text { jet }} \geq 2$ & 0.19 & 6.9 & $6.0_{-1.3}^{+2.2}$ & 0.69 & 0.35 \\
\hline \multirow{3}{*}{$N_{\text {jet }}^{80} \geq 7$} & $N_{b-\text { jet }} \geq 0$ & 3.1 & 110 & $130_{-30}^{+40}$ & 0.27 & 0.50 \\
\hline & $N_{b \text {-jet }} \geq 1$ & 2.7 & 100 & $120_{-30}^{+40}$ & 0.23 & 0.50 \\
\hline & $N_{b-\text { jet }} \geq 2$ & 1.7 & 60 & $72_{-17}^{+22}$ & 0.26 & 0.50 \\
\hline \multirow{3}{*}{$N_{\text {jet }}^{80} \geq 8$} & $N_{b \text {-jet }} \geq 0$ & 0.80 & 29 & $27_{-7}^{+10}$ & 0.60 & 0.40 \\
\hline & $N_{b-\mathrm{jet}} \geq 1$ & 0.62 & 22 & $24_{-7}^{+9}$ & 0.40 & 0.50 \\
\hline & $N_{b \text {-jet }} \geq 2$ & 0.49 & 18 & $16_{-5}^{+6}$ & 0.59 & 0.41 \\
\hline \multirow{3}{*}{$N_{\text {jet }}^{80} \geq 9$} & $N_{b \text {-jet }} \geq 0$ & 0.22 & 7.8 & $7.9_{-2.0}^{+3.4}$ & 0.47 & 0.50 \\
\hline & $N_{b-\text { jet }} \geq 1$ & 0.21 & 7.5 & $7.5_{-2.1}^{+2.8}$ & 0.48 & 0.50 \\
\hline & $N_{b \text {-jet }} \geq 2$ & 0.22 & 8.0 & $\begin{array}{r}5.9_{-1.4}^{+2.6} \\
\end{array}$ & 0.81 & 0.20 \\
\hline \multirow{2}{*}{$N_{\text {jet }}^{50} \geq 8$} & $M_{\mathrm{J}}^{\Sigma} \geq 340 \mathrm{GeV}$ & 2.9 & 100 & $130_{-30}^{+40}$ & 0.24 & 0.50 \\
\hline & $M_{\mathrm{J}}^{\Sigma} \geq 500 \mathrm{GeV}$ & 1.0 & 36 & $48_{-13}^{+17}$ & 0.18 & 0.50 \\
\hline \multirow{2}{*}{$N_{\text {jet }}^{50} \geq 9$} & $M_{\mathrm{J}}^{\Sigma} \geq 340 \mathrm{GeV}$ & 0.87 & 32 & $42_{-11}^{+14}$ & 0.17 & 0.50 \\
\hline & $M_{\mathrm{J}}^{\Sigma} \geq 500 \mathrm{GeV}$ & 0.32 & 12 & $20_{-6}^{+8}$ & 0.04 & 0.50 \\
\hline \multirow{2}{*}{$N_{\text {jet }}^{50} \geq 10$} & $M_{\mathrm{J}}^{\Sigma} \geq 340 \mathrm{GeV}$ & 0.25 & 9.1 & $14_{-4}^{+6}$ & 0.10 & 0.50 \\
\hline & $M_{\mathrm{J}}^{\Sigma} \geq 500 \mathrm{GeV}$ & 0.22 & 7.9 & $11_{-3}^{+4}$ & 0.18 & 0.50 \\
\hline
\end{tabular}

Table 5. Left to right: $95 \% \mathrm{CL}$ upper limits on the visible cross-section $\left(\langle\epsilon \sigma\rangle_{\text {obs }}^{95}\right)$ and on the number of signal events $\left(S_{\text {obs }}^{95}\right)$. The third column $\left(S_{\exp }^{95}\right)$ shows the $95 \%$ CL upper limit on the number of signal events, given the expected number (and $\pm 1 \sigma$ excursions on the expectation) of background events. The last two columns indicate $1-\mathrm{CL}_{\mathrm{b}}$, i.e. the complement of the $p$-value observed for the background-only hypothesis, and the discovery $p$-value $\left(p_{0}\right)$. 


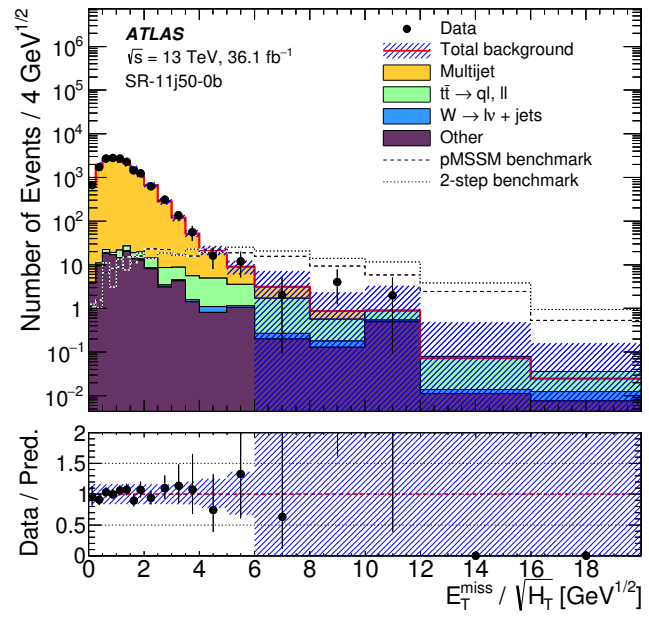

(a) $N_{\text {jet }}^{50} \geq 11, N_{b \text {-tag }} \geq 0$

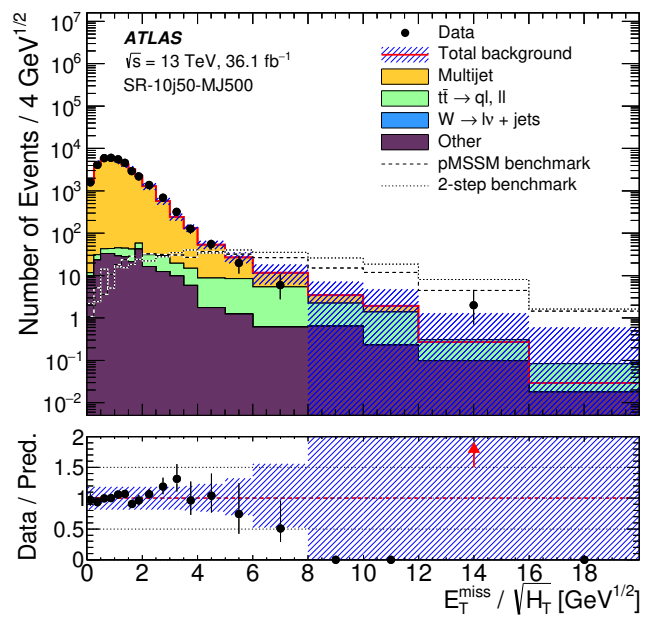

(b) $N_{\text {jet }}^{50} \geq 10, M_{\mathrm{J}}^{\Sigma}>500 \mathrm{GeV}$

Figure 5. Distributions of the $E_{\mathrm{T}}^{\mathrm{miss}} / \sqrt{H_{\mathrm{T}}}$ for events in the 11-jet $\mathrm{SR}$ for the $50 \mathrm{GeV}$ flavour channel, inclusive in $N_{b \text {-tag }}$ (a) and the 10-jet SR for the jet mass channel (b), with $M_{\mathrm{J}}^{\Sigma}>500 \mathrm{GeV}$. The backgrounds are scaled by the normalisation factors extracted from the fit, described in section 6.3. The blue hatched band indicates the quadrature sum of the statistical uncertainty from MC simulated samples and the various systematic uncertainties in the background prediction. The dashed lines labelled 'pMSSM' and '2-step' refer to benchmark signal points a pMSSM slice model with $\left(m_{\tilde{g}}, m_{\tilde{\chi}_{1}^{ \pm}}\right)=(1400,200) \mathrm{GeV}$ and a cascade decay model with $\left(m_{\tilde{g}}, m_{\tilde{\chi}_{1}^{0}}\right)=(1400,200) \mathrm{GeV}$. The lower panels show the ratio of the observed data to the total SM background. Red arrows indicate data points for which the error bar does not intersect the ratio plot.

In the $m_{\tilde{g}}, m_{\tilde{\chi}_{1}^{ \pm}}$projection of the pMSSM, constraints are set such that $m_{\tilde{g}} \lesssim 1600 \mathrm{GeV}$ is excluded for $m_{\tilde{\chi}_{1}^{ \pm}}<600 \mathrm{GeV}$. The limit falls to $m_{\tilde{g}} \lesssim 1520 \mathrm{GeV}$ for $m_{\tilde{\chi}_{1}^{ \pm}} \simeq 800 \mathrm{GeV}$.

Limits are set up to $m_{\tilde{g}} \approx 1800 \mathrm{GeV}$ for small LSP masses when considering the simplified model assuming a two-step cascade decay of the gluino. For $m_{\tilde{g}} \simeq 800 \mathrm{GeV}$, models are excluded provided that $m_{\tilde{\chi}_{1}^{0}}<475 \mathrm{GeV}$. The limits lie in the range $500<$ $m_{\tilde{\chi}_{1}^{0}}<700 \mathrm{GeV}$ as the gluino mass increases to $m_{\tilde{g}}=1600 \mathrm{GeV}$.

Simplified models of gluino-mediated top squark production are excluded for gluino masses up to $1500 \mathrm{GeV}$, as long as $m_{\tilde{\chi}_{1}^{0}} \lesssim 600 \mathrm{GeV}$, when assuming that the squark is more massive than the gluino. When RPC restrictions are removed, gluino masses between 625 and $1375 \mathrm{GeV}$ can be excluded, depending on the value of $m_{\tilde{t}_{1}}$, for $400<m_{\tilde{t}_{1}}<900 \mathrm{GeV}$ in a scenario where the top squarks decay through an RPV coupling to $\bar{s} \bar{b}$. 


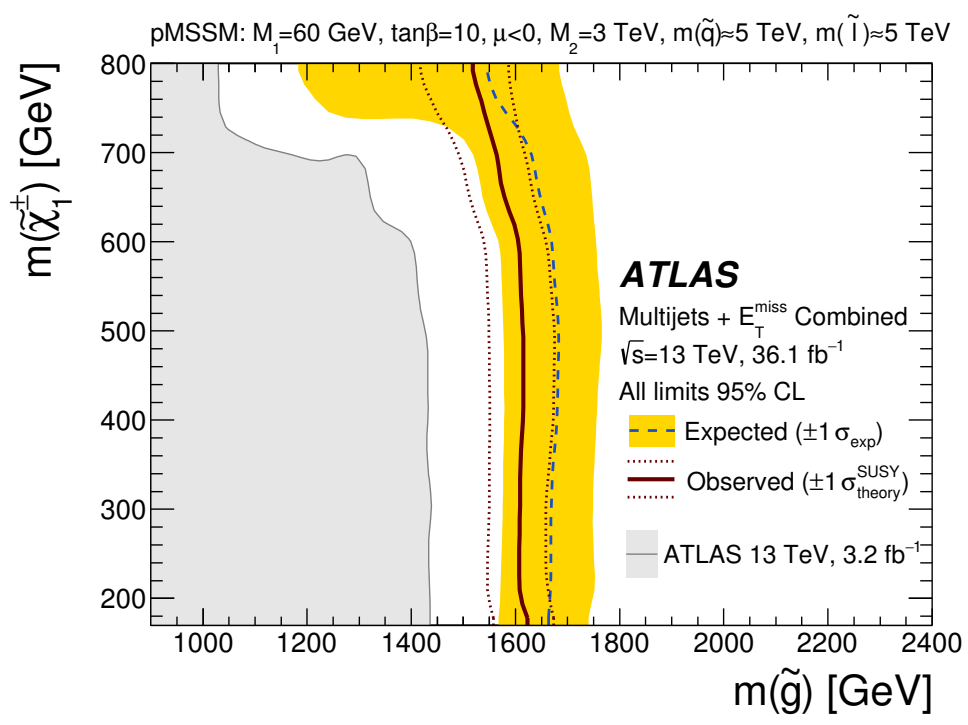

(a) pMSSM

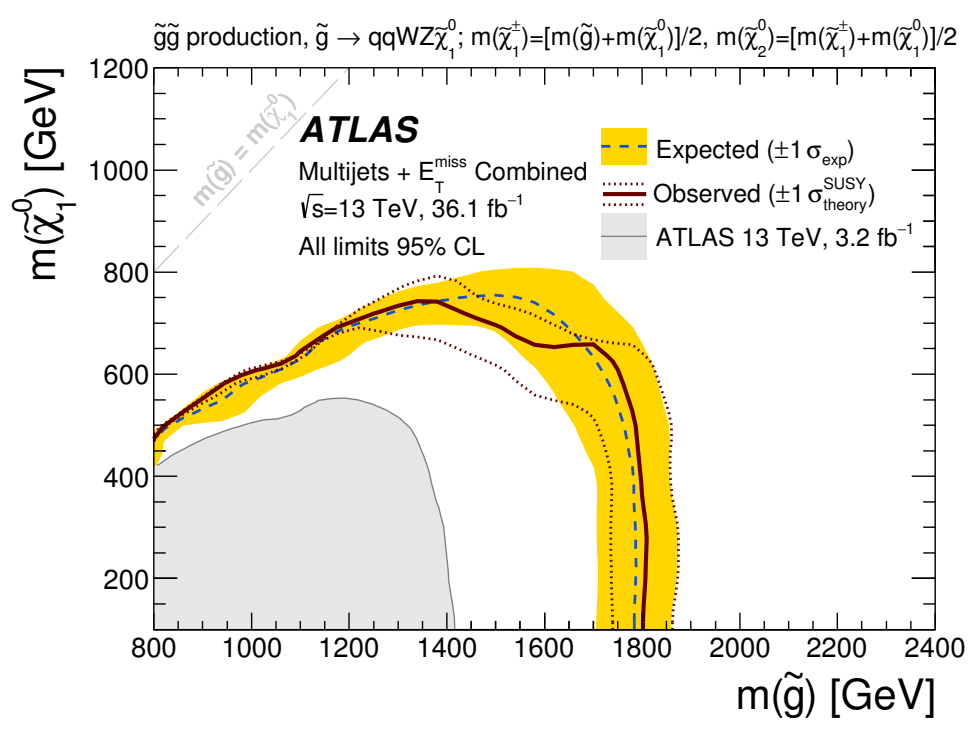

(b) Two-step decay

Figure 6. Exclusion contours in the $m_{\tilde{g}}, m_{\tilde{\chi}_{1}^{ \pm}}$plane for the pMSSM (a) and the $m_{\tilde{g}}, m_{\tilde{\chi}_{1}^{0}}$ plane in a simplified model with the gluino decaying via a two-step cascade (b). The solid maroon line indicates the observed limit, while the dashed blue line shows the expected limit. Experimental, $\mathrm{MC}$ theoretical and statistical uncertainties are shown in the yellow band. Dotted maroon lines delimit the variation of the observed limit within the $\pm 1 \sigma$ uncertainties in the signal cross-section at NLO+NLL accuracy. 


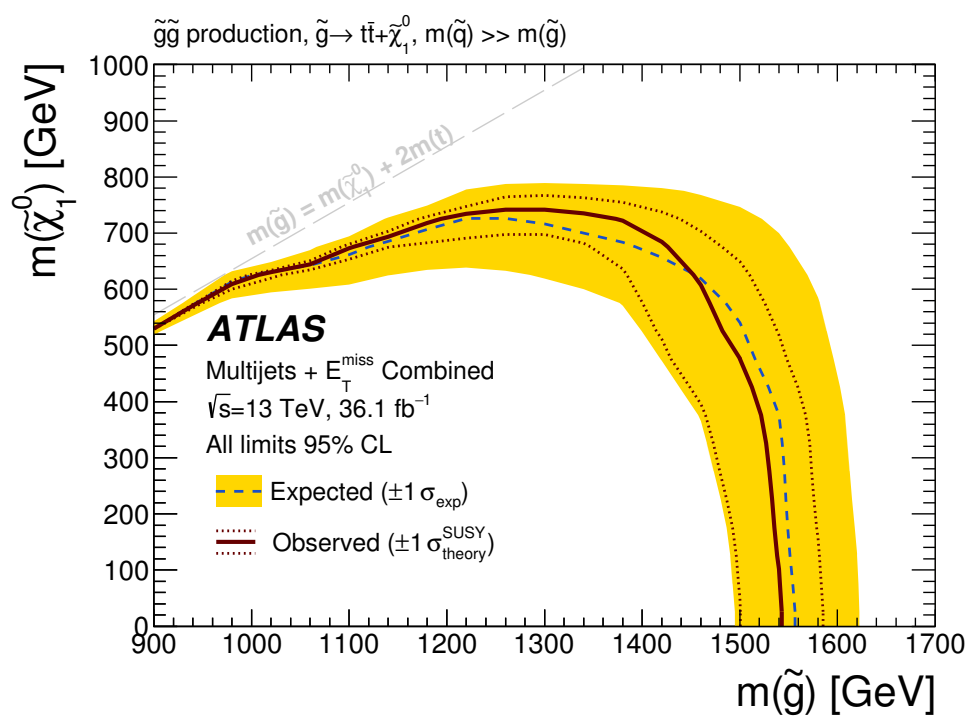

(a) Off-shell top squarks

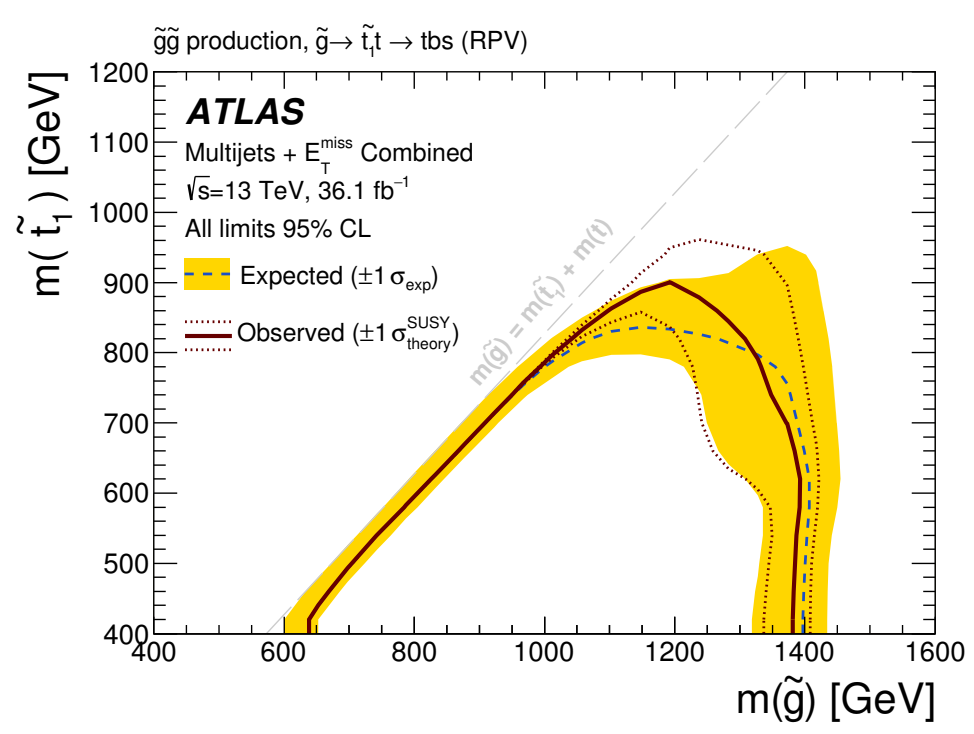

(b) RPV

Figure 7. Exclusion contours in gluino-mediated top squark production scenarios, illustrated in the $m_{\tilde{g}}, m_{\tilde{\chi}_{1}^{0}}$ plane with off-shell squarks (a) and an $R$-parity-violating scenario plane in which the top squark decays via $\tilde{t}_{1} \rightarrow s b(\mathrm{~b})$, shown in the $m_{\tilde{g}}, m_{\tilde{t}_{1}}$ plane. The solid maroon line indicates the observed limit, while the dashed blue line shows the expected limit. Experimental, MC theoretical and statistical uncertainties are shown in the yellow band. Dotted maroon lines delimit the variation of the observed limit within the $\pm 1 \sigma$ uncertainties in the signal cross-section at NLO+NLL accuracy. 


\section{Conclusion}

A search for heavy particles predicted by supersymmetric or other models of physics beyond the SM, that decay to produce large jet multiplicities in association with $E_{\mathrm{T}}^{\text {miss }}$, was performed using $36.1 \mathrm{fb}^{-1}$ of $\sqrt{s}=13 \mathrm{TeV}$ LHC $p p$ collision data collected by ATLAS in 2015 and 2016. No significant excesses over the Standard Model background were observed in signal regions selecting up to 11 jets with $p_{\mathrm{T}}>50 \mathrm{GeV}$ or 9 jets with $p_{\mathrm{T}}>80 \mathrm{GeV}$. The largest-jet-multiplicity event observed in data had 13 jets with $p_{\mathrm{T}}>80 \mathrm{GeV}$, while the greatest observed jet mass sum was $M_{\mathrm{J}}^{\Sigma}=1.3 \mathrm{TeV}$.

Exclusion limits are placed on gluino production in supersymmetric signal scenarios with a range of model assumptions. The tightest limits are set at $m_{\tilde{g}} \approx 1800 \mathrm{GeV}$ in a simplified model assuming a two-step cascade decay via the $\tilde{\chi}_{2}^{0}$ and $\tilde{\chi}_{1}^{ \pm}$. A slice of the phenomenological MSSM is excluded for $m_{\tilde{g}}<1520 \mathrm{GeV}$, with tighter constraints at $m_{\tilde{g}} \approx$ $1600 \mathrm{GeV}$ for $m_{\tilde{\chi}_{1}^{ \pm}}<600 \mathrm{GeV}$. When assuming that the gluino decays through off-shell top squarks, masses of the gluino below $1500 \mathrm{GeV}$ are excluded provided $m_{\tilde{\chi}_{1}^{0}}<600 \mathrm{GeV}$. Limits are also set in an R-parity-violating model with baryon-number-violating couplings permitting $\tilde{t}_{1} \rightarrow \bar{s} \bar{b}$, such that the gluino mass must be greater than $1200-1375 \mathrm{GeV}$ when the top squark mass is in the range $400<m_{\tilde{t}_{1}}<900 \mathrm{GeV}$ and $m_{\tilde{g}}-\left(m_{\tilde{t}_{1}}+m_{t}\right)>50 \mathrm{GeV}$.

\section{Acknowledgments}

We thank CERN for the very successful operation of the LHC, as well as the support staff from our institutions without whom ATLAS could not be operated efficiently.

We acknowledge the support of ANPCyT, Argentina; YerPhI, Armenia; ARC, Australia; BMWFW and FWF, Austria; ANAS, Azerbaijan; SSTC, Belarus; CNPq and FAPESP, Brazil; NSERC, NRC and CFI, Canada; CERN; CONICYT, Chile; CAS, MOST and NSFC, China; COLCIENCIAS, Colombia; MSMT CR, MPO CR and VSC CR, Czech Republic; DNRF and DNSRC, Denmark; IN2P3-CNRS, CEA-DSM/IRFU, France; SRNSF, Georgia; BMBF, HGF, and MPG, Germany; GSRT, Greece; RGC, Hong Kong SAR, China; ISF, I-CORE and Benoziyo Center, Israel; INFN, Italy; MEXT and JSPS, Japan; CNRST, Morocco; NWO, Netherlands; RCN, Norway; MNiSW and NCN, Poland; FCT, Portugal; MNE/IFA, Romania; MES of Russia and NRC KI, Russian Federation; JINR; MESTD, Serbia; MSSR, Slovakia; ARRS and MIZŠ, Slovenia; DST/NRF, South Africa; MINECO, Spain; SRC and Wallenberg Foundation, Sweden; SERI, SNSF and Cantons of Bern and Geneva, Switzerland; MOST, Taiwan; TAEK, Turkey; STFC, United Kingdom; DOE and NSF, United States of America. In addition, individual groups and members have received support from BCKDF, the Canada Council, CANARIE, CRC, Compute Canada, FQRNT, and the Ontario Innovation Trust, Canada; EPLANET, ERC, ERDF, FP7, Horizon 2020 and Marie Skłodowska-Curie Actions, European Union; Investissements d'Avenir Labex and Idex, ANR, Région Auvergne and Fondation Partager le Savoir, France; DFG and AvH Foundation, Germany; Herakleitos, Thales and Aristeia programmes co-financed by EU-ESF and the Greek NSRF; BSF, GIF and Minerva, Israel; 
BRF, Norway; CERCA Programme Generalitat de Catalunya, Generalitat Valenciana, Spain; the Royal Society and Leverhulme Trust, United Kingdom.

The crucial computing support from all WLCG partners is acknowledged gratefully, in particular from CERN, the ATLAS Tier-1 facilities at TRIUMF (Canada), NDGF (Denmark, Norway, Sweden), CC-IN2P3 (France), KIT/GridKA (Germany), INFN-CNAF (Italy), NL-T1 (Netherlands), PIC (Spain), ASGC (Taiwan), RAL (U.K.) and BNL (U.S.A.), the Tier-2 facilities worldwide and large non-WLCG resource providers. Major contributors of computing resources are listed in ref. [105].

Open Access. This article is distributed under the terms of the Creative Commons Attribution License (CC-BY 4.0), which permits any use, distribution and reproduction in any medium, provided the original author(s) and source are credited.

\section{References}

[1] L. Evans and P. Bryant, LHC Machine, 2008 JINST 3 S08001 [inSPIRE].

[2] Yu. A. Golfand and E.P. Likhtman, Extension of the algebra of Poincaré Group generators and violation of $p$ invariance, JETP Lett. 13 (1971) 323 [INSPIRE].

[3] D.V. Volkov and V.P. Akulov, Is the Neutrino a Goldstone Particle?, Phys. Lett. B 46 (1973) 109 [INSPIRE].

[4] J. Wess and B. Zumino, Supergauge Transformations in Four-Dimensions, Nucl. Phys. B 70 (1974) 39 [INSPIRE].

[5] J. Wess and B. Zumino, Supergauge Invariant Extension of Quantum Electrodynamics, Nucl. Phys. B 78 (1974) 1 [inSPIRE].

[6] S. Ferrara and B. Zumino, Supergauge Invariant Yang-Mills Theories, Nucl. Phys. B 79 (1974) 413 [INSPIRE].

[7] A. Salam and J.A. Strathdee, Supersymmetry and Nonabelian Gauges, Phys. Lett. B 51 (1974) 353 [INSPIRE].

[8] G.R. Farrar and P. Fayet, Phenomenology of the Production, Decay and Detection of New Hadronic States Associated with Supersymmetry, Phys. Lett. B 76 (1978) 575 [InSPIRE].

[9] S. Wolfram, Abundances of Stable Particles Produced in the Early Universe, Phys. Lett. B 82 (1979) 65 [INSPIRE].

[10] J. Rich, D. Lloyd Owen and M. Spiro, Experimental particle physics without accelerators, Phys. Rept. 151 (1987) 239 [INSPIRE].

[11] P.F. Smith, Terrestrial Searches for New Stable Particles, Contemp. Phys. 29 (1988) 159 [INSPIRE].

[12] S. Abel and T. Falk, Charge and color breaking in the constrained MSSM, Phys. Lett. B 444 (1998) 427 [hep-ph/9810297] [INSPIRE].

[13] H.K. Dreiner, An introduction to explicit R-parity violation, hep-ph/9707435 [INSPIRE].

[14] ATLAS collaboration, The ATLAS Experiment at the CERN Large Hadron Collider, 2008 JINST 3 S08003 [INSPIRE].

[15] T. Cohen, E. Izaguirre, M. Lisanti and H.K. Lou, Jet substructure by accident, JHEP 03 (2013) 161 [arXiv: 1212.1456] [INSPIRE]. 
[16] ATLAS collaboration, Search for new phenomena in final states with large jet multiplicities and missing transverse momentum using $\sqrt{s}=7 \mathrm{TeV}$ pp collisions with the ATLAS detector, JHEP 11 (2011) 099 [arXiv:1110.2299] [INSPIRE].

[17] ATLAS collaboration, Hunt for new phenomena using large jet multiplicities and missing transverse momentum with ATLAS in $4.7 \mathrm{fb}^{-1}$ of $\sqrt{s}=7 \mathrm{TeV}$ proton-proton collisions, JHEP 07 (2012) 167 [arXiv:1206.1760] [INSPIRE].

[18] ATLAS collaboration, Search for new phenomena in final states with large jet multiplicities and missing transverse momentum at $\sqrt{s}=8$ TeV proton-proton collisions using the ATLAS experiment, JHEP 10 (2013) 130 [Erratum ibid. 01 (2014) 109] [arXiv:1308.1841] [INSPIRE].

[19] ATLAS collaboration, Search for new phenomena in final states with large jet multiplicities and missing transverse momentum with ATLAS using $\sqrt{s}=13$ TeV proton-proton collisions, Phys. Lett. B 757 (2016) 334 [arXiv: 1602.06194] [INSPIRE].

[20] ATLAS collaboration, Summary of the searches for squarks and gluinos using $\sqrt{s}=8 \mathrm{TeV}$ pp collisions with the ATLAS experiment at the LHC, JHEP 10 (2015) 054 [arXiv: 1507.05525] [INSPIRE].

[21] ATLAS collaboration, Summary of the ATLAS experiment's sensitivity to supersymmetry after LHC Run 1 - interpreted in the phenomenological MSSM, JHEP 10 (2015) 134 [arXiv:1508.06608] [INSPIRE].

[22] ATLAS collaboration, Search for squarks and gluinos in final states with jets and missing transverse momentum at $\sqrt{s}=13 \mathrm{TeV}$ with the ATLAS detector, Eur. Phys. J. C 76 (2016) 392 [arXiv: 1605.03814] [INSPIRE].

[23] ATLAS collaboration, Search for gluinos in events with an isolated lepton, jets and missing transverse momentum at $\sqrt{s}=13 \mathrm{TeV}$ with the ATLAS detector, Eur. Phys. J. C 76 (2016) 565 [arXiv: 1605.04285] [INSPIRE].

[24] ATLAS collaboration, Search for pair production of gluinos decaying via stop and sbottom in events with $b$-jets and large missing transverse momentum in pp collisions at $\sqrt{s}=13$ TeV with the ATLAS detector, Phys. Rev. D 94 (2016) 032003 [arXiv: 1605. 09318] [INSPIRE].

[25] CMS collaboration, Search for supersymmetry in pp collisions at $\sqrt{s}=8 \mathrm{TeV}$ in events with a single lepton, large jet multiplicity and multiple b jets, Phys. Lett. B 733 (2014) 328 [arXiv:1311.4937] [INSPIRE].

[26] CMS collaboration, Search for stealth supersymmetry in events with jets, either photons or leptons and low missing transverse momentum in pp collisions at $8 \mathrm{TeV}$, Phys. Lett. B 743 (2015) 503 [arXiv: 1411.7255] [INSPIRE].

[27] CMS collaboration, Searches for supersymmetry based on events with $b$ jets and four $W$ bosons in pp collisions at $8 \mathrm{TeV}$, Phys. Lett. B $\mathbf{7 4 5}$ (2015) 5 [arXiv:1412.4109] [InSPIRE].

[28] CMS collaboration, Search for supersymmetry in the multijet and missing transverse momentum final state in pp collisions at 13 TeV, Phys. Lett. B 758 (2016) 152 [arXiv: 1602.06581] [INSPIRE].

[29] CMS collaboration, Inclusive search for supersymmetry using razor variables in $p p$ collisions at $\sqrt{s}=13 \mathrm{TeV}$, Phys. Rev. D 95 (2017) 012003 [arXiv: 1609.07658] [INSPIRE]. 
[30] CMS collaboration, A search for new phenomena in pp collisions at $\sqrt{s}=13 \mathrm{TeV}$ in final states with missing transverse momentum and at least one jet using the $\alpha_{\mathrm{T}}$ variable, Eur. Phys. J. C 77 (2017) 294 [arXiv:1611.00338] [InSPIRE].

[31] CMS collaboration, Search for supersymmetry in pp collisions at $\sqrt{s}=13 \mathrm{TeV}$ in the single-lepton final state using the sum of masses of large-radius jets, JHEP 08 (2016) 122 [arXiv: 1605.04608] [INSPIRE].

[32] CMS collaboration, Phenomenological MSSM interpretation of CMS searches in $p p$ collisions at $\sqrt{s}=7$ and $8 \mathrm{TeV}$, JHEP 10 (2016) 129 [arXiv:1606.03577] [INSPIRE].

[33] CMS collaboration, Search for supersymmetry in the all-hadronic final state using top quark tagging in pp collisions at $\sqrt{s}=13$ TeV, Phys. Rev. D 96 (2017) 012004 [arXiv: 1701.01954] [INSPIRE].

[34] CMS collaboration, Search for new phenomena with the $M_{\mathrm{T} 2}$ variable in the all-hadronic final state produced in proton-proton collisions at $\sqrt{s}=13$ TeV, Eur. Phys. J. C 77 (2017) 710 [arXiv: 1705.04650] [INSPIRE].

[35] CMS collaboration, Search for direct production of supersymmetric partners of the top quark in the all-jets final state in proton-proton collisions at $\sqrt{s}=13 \mathrm{TeV}, \mathrm{JHEP} 10$ (2017) 005 [arXiv: 1707.03316] [INSPIRE].

[36] ATLAS collaboration, Performance of the ATLAS Trigger System in 2010, Eur. Phys. J. C 72 (2012) 1849 [arXiv:1110.1530] [INSPIRE].

[37] ATLAS collaboration, Performance of the ATLAS Trigger System in 2015, Eur. Phys. J. C 77 (2017) 317 [arXiv:1611.09661] [INSPIRE].

[38] ATLAS collaboration, Luminosity determination in pp collisions at $\sqrt{s}=8 \mathrm{TeV}$ using the ATLAS detector at the LHC, Eur. Phys. J. C 76 (2016) 653 [arXiv:1608. 03953] [INSPIRE].

[39] ATLAS collaboration, Characterisation and mitigation of beam-induced backgrounds observed in the ATLAS detector during the 2011 proton-proton run, 2013 JINST 8 P07004 [arXiv: 1303.0223] [INSPIRE].

[40] ATLAS collaboration, Selection of jets produced in $13 \mathrm{TeV}$ proton-proton collisions with the ATLAS detector, ATLAS-CONF-2015-029 [INSPIRE].

[41] T. Sjöstrand, S. Mrenna and P.Z. Skands, A brief introduction to PYTHIA 8.1, Comput. Phys. Commun. 178 (2008) 852 [arXiv:0710.3820] [INSPIRE].

[42] ATLAS collaboration, Summary of ATLAS Pythia 8 tunes, ATL-PHYS-PUB-2012-003 [INSPIRE].

[43] A.D. Martin, W.J. Stirling, R.S. Thorne and G. Watt, Parton distributions for the LHC, Eur. Phys. J. C 63 (2009) 189 [arXiv:0901.0002] [InSPIRE].

[44] ATLAS collaboration, The ATLAS Simulation Infrastructure, Eur. Phys. J. C 70 (2010) 823 [arXiv: 1005.4568] [INSPIRE].

[45] GEANT4 collaboration, S. Agostinelli et al., GEANT4 - A simulation toolkit, Nucl. Instrum. Meth. A 506 (2003) 250 [inSPIRE].

[46] ATLAS collaboration, Performance of the Fast ATLAS Tracking Simulation (FATRAS) and the ATLAS Fast Calorimeter Simulation (FastCaloSim) with single particles, ATL-SOFT-PUB-2014-01 (2014). 
[47] P. Nason, A new method for combining NLO QCD with shower Monte Carlo algorithms, JHEP 11 (2004) 040 [hep-ph/0409146] [INSPIRE].

[48] S. Frixione, P. Nason and C. Oleari, Matching NLO QCD computations with Parton Shower simulations: the POWHEG method, JHEP 11 (2007) 070 [arXiv: 0709. 2092] [INSPIRE].

[49] S. Alioli, P. Nason, C. Oleari and E. Re, A general framework for implementing NLO calculations in shower Monte Carlo programs: the POWHEG BOX, JHEP 06 (2010) 043 [arXiv: 1002.2581] [INSPIRE].

[50] S. Alioli, P. Nason, C. Oleari and E. Re, NLO single-top production matched with shower in POWHEG: s- and t-channel contributions, JHEP 09 (2009) 111 [Erratum ibid. 02 (2010) 011] [arXiv:0907.4076] [INSPIRE].

[51] E. Re, Single-top Wt-channel production matched with parton showers using the POWHEG method, Eur. Phys. J. C 71 (2011) 1547 [arXiv:1009.2450] [InSPIRE].

[52] S. Alioli, S.-O. Moch and P. Uwer, Hadronic top-quark pair-production with one jet and parton showering, JHEP 01 (2012) 137 [arXiv:1110.5251] [INSPIRE].

[53] H.-L. Lai et al., New parton distributions for collider physics, Phys. Rev. D 82 (2010) 074024 [arXiv: 1007.2241] [INSPIRE].

[54] P. Artoisenet, R. Frederix, O. Mattelaer and R. Rietkerk, Automatic spin-entangled decays of heavy resonances in Monte Carlo simulations, JHEP 03 (2013) 015 [arXiv:1212.3460] [INSPIRE].

[55] T. Sjöstrand, S. Mrenna and P.Z. Skands, PYTHIA 6.4 Physics and Manual, JHEP 05 (2006) 026 [hep-ph/0603175] [INSPIRE].

[56] J. Pumplin, D.R. Stump, J. Huston, H.L. Lai, P.M. Nadolsky and W.K. Tung, New generation of parton distributions with uncertainties from global QCD analysis, JHEP $\mathbf{0 7}$ (2002) 012 [hep-ph/0201195] [INSPIRE].

[57] P.Z. Skands, Tuning Monte Carlo Generators: The Perugia Tunes, Phys. Rev. D 82 (2010) 074018 [arXiv: 1005.3457] [INSPIRE].

[58] D.J. Lange, The EvtGen particle decay simulation package, Nucl. Instrum. Meth. A 462 (2001) 152 [INSPIRE].

[59] M. Czakon and A. Mitov, Top++: a program for the calculation of the top-pair cross-section at hadron colliders, Comput. Phys. Commun. 185 (2014) 2930 [arXiv:1112.5675] [INSPIRE].

[60] J. Alwall et al., The automated computation of tree-level and next-to-leading order differential cross sections and their matching to parton shower simulations, JHEP $\mathbf{0 7}$ (2014) 079 [arXiv: 1405.0301] [INSPIRE].

[61] ATLAS collaboration, ATLAS Run 1 PYTHIA8 tunes, ATL-PHYS-PUB-2014-021 (2014).

[62] R.D. Ball et al., Parton distributions with LHC data, Nucl. Phys. B 867 (2013) 244 [arXiv: 1207.1303] [INSPIRE].

[63] NNPDF collaboration, R.D. Ball et al., Parton distributions for the LHC Run II, JHEP 04 (2015) 040 [arXiv: 1410.8849] [INSPIRE].

[64] T. Gleisberg and S. Hoeche, Comix, a new matrix element generator, JHEP 12 (2008) 039 [arXiv:0808.3674] [INSPIRE]. 
[65] F. Cascioli, P. Maierhofer and S. Pozzorini, Scattering Amplitudes with Open Loops, Phys. Rev. Lett. 108 (2012) 111601 [arXiv:1111.5206] [INSPIRE].

[66] S. Schumann and F. Krauss, A parton shower algorithm based on Catani-Seymour dipole factorisation, JHEP 03 (2008) 038 [arXiv:0709.1027] [INSPIRE].

[67] S. Hoeche, F. Krauss, M. Schonherr and F. Siegert, QCD matrix elements + parton showers: The NLO case, JHEP 04 (2013) 027 [arXiv:1207.5030] [INSPIRE].

[68] T. Gleisberg et al., Event generation with SHERPA 1.1, JHEP 02 (2009) 007 [arXiv:0811.4622] [INSPIRE].

[69] ATLAS collaboration, Comparison of Monte Carlo generator predictions from Powheg and Sherpa to ATLAS measurements of top pair production at $7 \mathrm{TeV}$, ATL-PHYS-PUB-2015-011 (2015).

[70] M. Bahr et al., HERWIG++ physics and manual, Eur. Phys. J. C 58 (2008) 639 [arXiv:0803.0883] [INSPIRE].

[71] ATLAS collaboration, Multi-boson simulation for $13 \mathrm{TeV}$ ATLAS analyses, ATL-PHYS-PUB-2016-002 (2016).

[72] ATLAS collaboration, Monte Carlo Generators for the Production of a $W$ or $Z / \gamma^{*}$ Boson in Association with Jets at ATLAS in Run 2, ATL-PHYS-PUB-2016-003 (2016).

[73] ATLAS collaboration, Simulation of top quark production for the ATLAS experiment at $\sqrt{s}=13 \mathrm{Te} V$, ATL-PHYS-PUB-2016-004 (2016).

[74] ATLAS collaboration, Modelling of the $t \bar{t} H$ and $t \bar{t} V(V=W, Z)$ processes for $\sqrt{s}=13 \mathrm{TeV}$ ATLAS analyses, ATL-PHYS-PUB-2016-005 (2016).

[75] MSSM Working Group collaboration, A. Djouadi et al., The minimal supersymmetric standard model: Group summary report, hep-ph/9901246 [INSPIRE].

[76] C.F. Berger, J.S. Gainer, J.L. Hewett and T.G. Rizzo, Supersymmetry without prejudice, JHEP 02 (2009) 023 [arXiv:0812.0980] [INSPIRE].

[77] B.C. Allanach, SOFTSUSY: a program for calculating supersymmetric spectra, Comput. Phys. Commun. 143 (2002) 305 [hep-ph/0104145] [INSPIRE].

[78] A. Djouadi, M.M. Muhlleitner and M. Spira, Decays of supersymmetric particles: The Program SUSY-HIT (SUspect-SdecaY-HDECAY-InTerface), Acta Phys. Polon. B 38 (2007) 635 [hep-ph/0609292] [INSPIRE].

[79] L. Lönnblad, Correcting the color dipole cascade model with fixed order matrix elements, JHEP 05 (2002) 046 [hep-ph/0112284] [INSPIRE].

[80] W. Beenakker, R. Hopker, M. Spira and P.M. Zerwas, Squark and gluino production at hadron colliders, Nucl. Phys. B 492 (1997) 51 [hep-ph/9610490] [InSPIRE].

[81] A. Kulesza and L. Motyka, Threshold resummation for squark-antisquark and gluino-pair production at the LHC, Phys. Rev. Lett. 102 (2009) 111802 [arXiv:0807.2405] [INSPIRE].

[82] A. Kulesza and L. Motyka, Soft gluon resummation for the production of gluino-gluino and squark-antisquark pairs at the LHC, Phys. Rev. D 80 (2009) 095004 [arXiv: 0905.4749] [INSPIRE].

[83] W. Beenakker, S. Brensing, M. Krämer, A. Kulesza, E. Laenen and I. Niessen, Soft-gluon resummation for squark and gluino hadroproduction, JHEP 12 (2009) 041 [arXiv:0909.4418] [INSPIRE]. 
[84] W. Beenakker et al., Squark and Gluino Hadroproduction, Int. J. Mod. Phys. A 26 (2011) 2637 [arXiv: 1105.1110] [INSPIRE].

[85] C. Borschensky et al., Squark and gluino production cross sections in pp collisions at $\sqrt{s}=13,14,33$ and $100 \mathrm{TeV}$, Eur. Phys. J. C 74 (2014) 3174 [arXiv:1407.5066] [INSPIRE].

[86] ATLAS collaboration, Vertex Reconstruction Performance of the ATLAS Detector at $\sqrt{s}=13 \mathrm{TeV}$, ATL-PHYS-PUB-2015-026 (2015).

[87] ATLAS collaboration, Topological cell clustering in the ATLAS calorimeters and its performance in LHC Run 1, Eur. Phys. J. C 77 (2017) 490 [arXiv:1603.02934] [INSPIRE].

[88] M. Cacciari, G.P. Salam and G. Soyez, The anti-k $k_{t}$ jet clustering algorithm, JHEP 04 (2008) 063 [arXiv: 0802.1189] [INSPIRE].

[89] M. Cacciari, G.P. Salam and G. Soyez, FastJet user manual, Eur. Phys. J. C 72 (2012) 1896 [arXiv:1111.6097] [INSPIRE].

[90] M. Cacciari and G.P. Salam, Pileup subtraction using jet areas, Phys. Lett. B 659 (2008) 119 [arXiv:0707.1378] [inSPIRE].

[91] ATLAS collaboration, Jet energy scale measurements and their systematic uncertainties in proton-proton collisions at $\sqrt{s}=13 \mathrm{TeV}$ with the ATLAS detector, Phys. Rev. D 96 (2017) 072002 [arXiv: 1703.09665] [INSPIRE].

[92] ATLAS collaboration, Performance of pile-up mitigation techniques for jets in pp collisions at $\sqrt{s}=8 \mathrm{TeV}$ using the ATLAS detector, Eur. Phys. J. C 76 (2016) 581 [arXiv: 1510.03823] [INSPIRE].

[93] ATLAS collaboration, Optimisation of the ATLAS b-tagging performance for the 2016 LHC Run, ATL-PHYS-PUB-2016-012 (2016).

[94] ATLAS collaboration, Performance of b-Jet Identification in the ATLAS Experiment, 2016 JINST 11 P04008 [arXiv: 1512.01094] [INSPIRE].

[95] B. Nachman, P. Nef, A. Schwartzman, M. Swiatlowski and C. Wanotayaroj, Jets from Jets: Re-clustering as a tool for large radius jet reconstruction and grooming at the LHC, JHEP 02 (2015) 075 [arXiv: 1407.2922] [INSPIRE].

[96] ATLAS collaboration, Electron efficiency measurements with the ATLAS detector using 2012 LHC proton-proton collision data, Eur. Phys. J. C 77 (2017) 195 [arXiv:1612.01456] [INSPIRE].

[97] ATLAS collaboration, Measurement of the photon identification efficiencies with the ATLAS detector using LHC Run-1 data, Eur. Phys. J. C 76 (2016) 666 [arXiv: 1606. 01813] [INSPIRE].

[98] ATLAS collaboration, Electron and photon energy calibration with the ATLAS detector using LHC Run 1 data, Eur. Phys. J. C 74 (2014) 3071 [arXiv:1407.5063] [InSPIRE].

[99] ATLAS collaboration, Muon reconstruction performance of the ATLAS detector in proton-proton collision data at $\sqrt{s}=13$ TeV, Eur. Phys. J. C 76 (2016) 292 [arXiv: 1603. 05598] [INSPIRE].

[100] ATLAS collaboration, Muon reconstruction performance in early $\sqrt{s}=13 \mathrm{TeV}$ data, ATL-PHYS-PUB-2015-037 (2015). 
[101] ATLAS collaboration, Performance of algorithms that reconstruct missing transverse momentum in $\sqrt{s}=8 \mathrm{TeV}$ proton-proton collisions in the ATLAS detector, Eur. Phys. J. C 77 (2017) 241 [arXiv: 1609.09324] [INSPIRE].

[102] M. Baak, G.J. Besjes, D. Côte, A. Koutsman, J. Lorenz and D. Short, HistFitter software framework for statistical data analysis, Eur. Phys. J. C 75 (2015) 153 [arXiv:1410.1280] [INSPIRE].

[103] G. Cowan, K. Cranmer, E. Gross and O. Vitells, Asymptotic formulae for likelihood-based tests of new physics, Eur. Phys. J. C 71 (2011) 1554 [Erratum ibid. C 73 (2013) 2501] [arXiv: 1007.1727] [INSPIRE].

[104] A.L. Read, Presentation of search results: The CL(s) technique, J. Phys. G 28 (2002) 2693 [INSPIRE].

[105] ATLAS collaboration, ATLAS Computing Acknowledgements 2016-2017, ATL-GEN-PUB-2016-002 (2016). 


\section{The ATLAS collaboration}

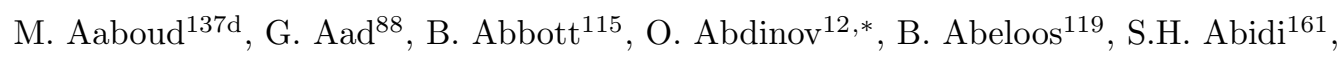
O.S. AbouZeid ${ }^{139}$, N.L. Abraham ${ }^{151}$, H. Abramowicz ${ }^{155}$, H. Abreu ${ }^{154}$, R. Abreu ${ }^{118}$, Y. Abulaiti ${ }^{148 a, 148 b}$, B.S. Acharya ${ }^{167 a, 167 b, a}$, S. Adachi ${ }^{157}$, L. Adamczyk ${ }^{41 a}$, J. Adelman ${ }^{110}$, M. Adersberger ${ }^{102}$, T. Adye ${ }^{133}$, A.A. Affolder ${ }^{139}$, Y. Afik ${ }^{154}$, T. Agatonovic-Jovin ${ }^{14}$, C. Agheorghiesei ${ }^{28 c}$, J.A. Aguilar-Saavedra ${ }^{128 a, 128 f}$, S.P. Ahlen ${ }^{24}$, F. Ahmadov ${ }^{68, b}$, G. Aielli ${ }^{135 a, 135 b}$, S. Akatsuka ${ }^{71}$, H. Akerstedt ${ }^{148 a, 148 b}$, T.P.A. Åkesson ${ }^{84}$, E. Akilli $^{52}$, A.V. Akimov ${ }^{98}$, G.L. Alberghi ${ }^{22 a, 22 b}$, J. Albert ${ }^{172}$, P. Albicocco ${ }^{50}$, M.J. Alconada Verzini ${ }^{74}$, S.C. Alderweireldt ${ }^{108}$, M. Aleksa ${ }^{32}$, I.N. Aleksandrov ${ }^{68}$, C. Alexa ${ }^{28 b}$, G. Alexander ${ }^{155}$, T. Alexopoulos ${ }^{10}$, M. Alhroob ${ }^{115}$, B. Ali ${ }^{130}$, M. Aliev ${ }^{76 a, 76 b}$, G. Alimonti ${ }^{94 a}$, J. Alison ${ }^{33}$, S.P. Alkire ${ }^{38}$, B.M.M. Allbrooke ${ }^{151}$, B.W. Allen ${ }^{118}$, P.P. Allport ${ }^{19}$, A. Aloisio 106a, 106b, A. Alonso ${ }^{39}$, F. Alonso ${ }^{74}$, C. Alpigiani ${ }^{140}$, A.A. Alshehri ${ }^{56}$, M.I. Alstaty ${ }^{88}$, B. Alvarez Gonzalez ${ }^{32}$, D. Álvarez Piqueras ${ }^{170}$, M.G. Alviggi ${ }^{106 a, 106 b}$, B.T. Amadio ${ }^{16}$, Y. Amaral Coutinho ${ }^{26 a}$, C. Amelung ${ }^{25}$, D. Amidei ${ }^{92}$, S.P. Amor Dos Santos ${ }^{128 a, 128 c}$, S. Amoroso ${ }^{32}$, G. Amundsen ${ }^{25}$, C. Anastopoulos ${ }^{141}$, L.S. Ancu ${ }^{52}$, N. Andari ${ }^{19}$, T. Andeen ${ }^{11}$, C.F. Anders ${ }^{60 b}$, J.K. Anders ${ }^{77}$, K.J. Anderson ${ }^{33}$, A. Andreazza ${ }^{94 a, 94 b}$, V. Andrei ${ }^{60 a}$, S. Angelidakis ${ }^{37}$, I. Angelozzi ${ }^{109}$, A. Angerami ${ }^{38}$, A.V. Anisenkov ${ }^{111, c}$, N. Anjos ${ }^{13}$, A. Annovi ${ }^{126 a, 126 b}$, C. Antel ${ }^{60 a}$, M. Antonelli ${ }^{50}$, A. Antonov ${ }^{100, *}$, D.J. Antrim ${ }^{166}$, F. Anulli ${ }^{134 a}$, M. Aoki ${ }^{69}$, L. Aperio Bella ${ }^{32}$, G. Arabidze ${ }^{93}$, Y. Arai ${ }^{69}$, J.P. Araque ${ }^{128 a}$, V. Araujo Ferraz ${ }^{26 a}$, A.T.H. Arce ${ }^{48}$, R.E. Ardell ${ }^{80}$, F.A. Arduh ${ }^{74}$, J-F. Arguin ${ }^{97}$, S. Argyropoulos ${ }^{66}$, M. Arik ${ }^{20 a}$, A.J. Armbruster ${ }^{32}$, L.J. Armitage ${ }^{79}$, O. Arnaez ${ }^{161}$,

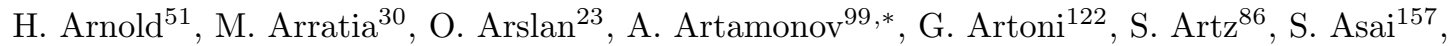
N. Asbah ${ }^{45}$, A. Ashkenazi ${ }^{155}$, L. Asquith ${ }^{151}$, K. Assamagan ${ }^{27}$, R. Astalos ${ }^{146 a}$, M. Atkinson ${ }^{169}$, N.B. Atlay ${ }^{143}$, K. Augsten ${ }^{130}$, G. Avolio ${ }^{32}$, B. Axen ${ }^{16}$, M.K. Ayoub ${ }^{119}$, G. Azuelos ${ }^{97, d}$,

A.E. Baas ${ }^{60 a}$, M.J. Baca ${ }^{19}$, H. Bachacou ${ }^{138}$, K. Bachas ${ }^{76 a, 76 b}$, M. Backes ${ }^{122}$, P. Bagnaia ${ }^{134 a, 134 b}$, M. Bahmani ${ }^{42}$, H. Bahrasemani ${ }^{144}$, J.T. Baines ${ }^{133}$, M. Bajic ${ }^{39}$, O.K. Baker ${ }^{179}$, E.M. Baldin ${ }^{111, c}$, P. Balek ${ }^{175}$, F. Balli ${ }^{138}$, W.K. Balunas ${ }^{124}$, E. Banas ${ }^{42}$, A. Bandyopadhyay ${ }^{23}$, Sw. Banerjee ${ }^{176, e}$, A.A.E. Bannoura ${ }^{178}$, L. Barak ${ }^{155}$, E.L. Barberio ${ }^{91}$, D. Barberis ${ }^{53 a, 53 b}$, M. Barbero ${ }^{88}$, T. Barillari ${ }^{103}$, M-S Barisits ${ }^{32}$, J.T. Barkeloo ${ }^{118}$, T. Barklow ${ }^{145}$, N. Barlow ${ }^{30}$, S.L. Barnes ${ }^{36 c}$, B.M. Barnett ${ }^{133}$, R.M. Barnett ${ }^{16}$, Z. Barnovska-Blenessy ${ }^{36 a}$, A. Baroncelli ${ }^{136 a}$, G. Barone ${ }^{25}$, A.J. Barr ${ }^{122}$, L. Barranco Navarro ${ }^{170}$, F. Barreiro ${ }^{85}$, J. Barreiro Guimarães da Costa ${ }^{35 a}$, R. Bartoldus ${ }^{145}$, A.E. Barton ${ }^{75}$, P. Bartos ${ }^{146 a}$, A. Basalaev ${ }^{125}$, A. Bassalat ${ }^{119, f}$, R.L. Bates ${ }^{56}$, S.J. Batista ${ }^{161}$, J.R. Batley ${ }^{30}$, M. Battaglia ${ }^{139}$, M. Bauce ${ }^{134 a, 134 b}$, F. Bauer ${ }^{138}$, H.S. Bawa ${ }^{145, g}$, J.B. Beacham ${ }^{113}$, M.D. Beattie ${ }^{75}$, T. Beau ${ }^{83}$, P.H. Beauchemin ${ }^{165}$, P. Bechtle ${ }^{23}$, H.P. Beck ${ }^{18, h}$, H.C. Beck ${ }^{57}$, K. Becker ${ }^{122}$, M. Becker ${ }^{86}$, C. Becot ${ }^{112}$, A.J. Beddall ${ }^{20 e}$, A. Beddall ${ }^{20 b}$, V.A. Bednyakov ${ }^{68}$, M. Bedognetti ${ }^{109}$, C.P. Bee ${ }^{150}$, T.A. Beermann ${ }^{32}$, M. Begalli ${ }^{26 a}$, M. Begel ${ }^{27}$, J.K. Behr ${ }^{45}$, A.S. Bell ${ }^{81}$, G. Bella ${ }^{155}$, L. Bellagamba ${ }^{22 a}$, A. Bellerive ${ }^{31}$, M. Bellomo ${ }^{154}$, K. Belotskiy ${ }^{100}$, O. Beltramello ${ }^{32}$, N.L. Belyaev ${ }^{100}$, O. Benary ${ }^{155, *}$, D. Benchekroun ${ }^{137 a}$, M. Bender ${ }^{102}$, K. Bendtz ${ }^{148 a, 148 b}$, N. Benekos ${ }^{10}$, Y. Benhammou ${ }^{155}$, E. Benhar Noccioli ${ }^{179}$, J. Benitez ${ }^{66}$, D.P. Benjamin ${ }^{48}$, M. Benoit ${ }^{52}$, J.R. Bensinger ${ }^{25}$, S. Bentvelsen ${ }^{109}$, L. Beresford ${ }^{122}$, M. Beretta ${ }^{50}$, D. Berge ${ }^{109}$, E. Bergeaas Kuutmann ${ }^{168}$, N. Berger ${ }^{5}$, J. Beringer ${ }^{16}$, S. Berlendis ${ }^{58}$, N.R. Bernard ${ }^{89}$, G. Bernardi ${ }^{83}$, C. Bernius ${ }^{145}$, F.U. Bernlochner ${ }^{23}$, T. Berry ${ }^{80}$, P. Berta ${ }^{86}$, C. Bertella ${ }^{35 a}$, G. Bertoli ${ }^{148 a, 148 b}$, F. Bertolucci ${ }^{126 a, 126 b}$, I.A. Bertram ${ }^{75}$, C. Bertsche ${ }^{45}$, D. Bertsche ${ }^{115}$, G.J. Besjes ${ }^{39}$, O. Bessidskaia Bylund ${ }^{148 a, 148 b}$, M. Bessner ${ }^{45}$, N. Besson ${ }^{138}$, A. Bethani ${ }^{87}$, S. Bethke ${ }^{103}$, A.J. Bevan ${ }^{79}$, J. Beyer ${ }^{103}$, R.M. Bianchi ${ }^{127}$, O. Biebel ${ }^{102}$, D. Biedermann ${ }^{17}$, R. Bielski ${ }^{87}$, K. Bierwagen ${ }^{86}$, N.V. Biesuz ${ }^{126 a, 126 b}$, M. Biglietti ${ }^{136 a}$, T.R.V. Billoud ${ }^{97}$, H. Bilokon ${ }^{50}$, M. Bindi ${ }^{57}$, A. Bingul ${ }^{20 b}$, C. Bini ${ }^{134 a, 134 b}$, S. Biondi ${ }^{22 a, 22 b}$, T. Bisanz ${ }^{57}$, C. Bittrich ${ }^{47}$, D.M. Bjergaard ${ }^{48}$, J.E. Black ${ }^{145}$, K.M. Black ${ }^{24}$, R.E. Blair ${ }^{6}$, 
T. Blazek ${ }^{146 a}$, I. Bloch ${ }^{45}$, C. Blocker ${ }^{25}$, A. Blue ${ }^{56}$, W. Blum ${ }^{86, *}$, U. Blumenschein ${ }^{79}$, S. Blunier ${ }^{34 a}$, G.J. Bobbink ${ }^{109}$, V.S. Bobrovnikov ${ }^{111, c}$, S.S. Bocchetta ${ }^{84}$, A. Bocci ${ }^{48}$, C. Bock ${ }^{102}$, M. Boehler ${ }^{51}$, D. Boerner ${ }^{178}$, D. Bogavac ${ }^{102}$, A.G. Bogdanchikov ${ }^{111}$, C. Bohm ${ }^{148 a}$, V. Boisvert ${ }^{80}$, P. Bokan ${ }^{168, i}$, T. Bold ${ }^{41 a}$, A.S. Boldyrev ${ }^{101}$, A.E. Bolz ${ }^{60 b}$, M. Bomben ${ }^{83}$, M. Bona ${ }^{79}$, M. Boonekamp ${ }^{138}$, A. Borisov ${ }^{132}$, G. Borissov ${ }^{75}$, J. Bortfeldt ${ }^{32}$, D. Bortoletto ${ }^{122}$, V. Bortolotto ${ }^{62 \mathrm{a}, 62 \mathrm{~b}, 62 \mathrm{c}}$, D. Boscherini22a, M. Bosman ${ }^{13}$, J.D. Bossio Sola ${ }^{29}$, J. Boudreau ${ }^{127}$, J. Bouffard ${ }^{2}$, E.V. Bouhova-Thacker ${ }^{75}$, D. Boumediene ${ }^{37}$, C. Bourdarios ${ }^{119}$, S.K. Boutle ${ }^{56}$, A. Boveia ${ }^{113}$, J. Boyd ${ }^{32}$, I.R. Boyko ${ }^{68}$, J. Bracinik ${ }^{19}$, A. Brandt ${ }^{8}$, G. Brandt ${ }^{57}$, O. Brandt ${ }^{60 a}$, U. Bratzler ${ }^{158}$, B. Brau ${ }^{89}$, J.E. Brau ${ }^{118}$, W.D. Breaden Madden ${ }^{56}$, K. Brendlinger ${ }^{45}$, A.J. Brennan ${ }^{91}$, L. Brenner ${ }^{109}$, R. Brenner ${ }^{168}$, S. Bressler ${ }^{175}$, D.L. Briglin ${ }^{19}$, T.M. Bristow ${ }^{49}$, D. Britton ${ }^{56}$, D. Britzger ${ }^{45}$, F.M. Brochu ${ }^{30}$, I. Brock ${ }^{23}$, R. Brock ${ }^{93}$, G. Brooijmans ${ }^{38}$, T. Brooks ${ }^{80}$, W.K. Brooks ${ }^{34 b}$, J. Brosamer ${ }^{16}$, E. Brost ${ }^{110}$, J.H Broughton ${ }^{19}$, P.A. Bruckman de Renstrom ${ }^{42}$, D. Bruncko ${ }^{146 b}$, A. Bruni ${ }^{22 a}$, G. Bruni ${ }^{22 a}$, L.S. Bruni ${ }^{109}$, S. Bruno ${ }^{135 a, 135 b}$, BH Brunt $^{30}$, M. Bruschi ${ }^{22 a}$, N. Bruscino ${ }^{23}$, P. Bryant ${ }^{33}$, L. Bryngemark ${ }^{45}$, T. Buanes ${ }^{15}$, Q. Buat ${ }^{144}$, P. Buchholz ${ }^{143}$, A.G. Buckley ${ }^{56}$, I.A. Budagov ${ }^{68}$, F. Buehrer ${ }^{51}$, M.K. Bugge ${ }^{121}$, O. Bulekov ${ }^{100}$, D. Bullock ${ }^{8}$, T.J. Burch ${ }^{110}$, S. Burdin ${ }^{77}$, C.D. Burgard ${ }^{51}$, A.M. Burger ${ }^{5}$, B. Burghgrave ${ }^{110}$, K. Burka ${ }^{42}$, S. Burke ${ }^{133}$, I. Burmeister ${ }^{46}$, J.T.P. Burr ${ }^{122}$, E. Busato ${ }^{37}$, D. Büscher ${ }^{51}$, V. Büscher ${ }^{86}$, P. Bussey ${ }^{56}$, J.M. Butler ${ }^{24}$, C.M. Buttar ${ }^{56}$, J.M. Butterworth ${ }^{81}$, P. Butti ${ }^{32}$, W. Buttinger ${ }^{27}$, A. Buzatu ${ }^{153}$, A.R. Buzykaev ${ }^{111, c}$, S. Cabrera Urbán ${ }^{170}$, D. Caforio ${ }^{130}$, V.M. Cairo ${ }^{40 a}, 40 b$, O. Cakir ${ }^{4 a}$, N. Calace ${ }^{52}$, P. Calafiura ${ }^{16}$, A. Calandri ${ }^{88}$, G. Calderini ${ }^{83}$, P. Calfayan ${ }^{64}$, G. Callea ${ }^{40 a, 40 b}$, L.P. Caloba ${ }^{26 a}$, S. Calvente Lopez ${ }^{85}$, D. Calvet ${ }^{37}$, S. Calvet ${ }^{37}$, T.P. Calvet ${ }^{88}$, R. Camacho Toro ${ }^{33}$, S. Camarda ${ }^{32}$, P. Camarri ${ }^{135 a, 135 b}$, D. Cameron ${ }^{121}$, R. Caminal Armadans ${ }^{169}$, C. Camincher ${ }^{58}$, S. Campana ${ }^{32}$, M. Campanelli ${ }^{81}$, A. Camplani ${ }^{94 a, 94 b}$, A. Campoverde ${ }^{143}$, V. Canale ${ }^{106 a, 106 b}$, M. Cano Bret ${ }^{36 c}$, J. Cantero $^{116}$, T. Cao ${ }^{155}$, M.D.M. Capeans Garrido ${ }^{32}$, I. Caprini ${ }^{28 b}$, M. Caprini ${ }^{28 b}$, M. Capua ${ }^{40 a, 40 b}$, R.M. Carbone ${ }^{38}$, R. Cardarelli ${ }^{135 a}$, F. Cardillo ${ }^{51}$, I. Carli ${ }^{131}$, T. Carli ${ }^{32}$, G. Carlino ${ }^{106 a}$, B.T. Carlson ${ }^{127}$, L. Carminati ${ }^{94 a, 94 b}$, R.M.D. Carney ${ }^{148 a, 148 b}$, S. Caron ${ }^{108}$, E. Carquin ${ }^{34 b}$, S. Carrá94a,94b, G.D. Carrillo-Montoya ${ }^{32}$, D. Casadei ${ }^{19}$, M.P. Casado ${ }^{13, j}$, M. Casolino ${ }^{13}$, D.W. Casper ${ }^{166}$, R. Castelijn ${ }^{109}$, V. Castillo Gimenez ${ }^{170}$, N.F. Castro ${ }^{128 a}, k$, A. Catinaccio ${ }^{32}$, J.R. Catmore ${ }^{121}$, A. Cattai ${ }^{32}$, J. Caudron ${ }^{23}$, V. Cavaliere ${ }^{169}$, E. Cavallaro ${ }^{13}$, D. Cavalli ${ }^{94 a}$, M. Cavalli-Sforza ${ }^{13}$, V. Cavasinni ${ }^{126 a, 126 b}$, E. Celebi ${ }^{20 d}$, F. Ceradini ${ }^{136 a, 136 b}$, L. Cerda Alberich ${ }^{170}$, A.S. Cerqueira ${ }^{26 b}$, A. Cerri ${ }^{151}$, L. Cerrito ${ }^{135 a, 135 b}$, F. Cerutti ${ }^{16}$, A. Cervelli ${ }^{18}$, S.A. Cetin ${ }^{20 d}$, A. Chafaq ${ }^{137 a}$, D. Chakraborty ${ }^{110}$, S.K. Chan ${ }^{59}$, W.S. Chan ${ }^{109}$, Y.L. Chan ${ }^{62 a}$, P. Chang ${ }^{169}$, J.D. Chapman ${ }^{30}$, D.G. Charlton ${ }^{19}$, C.C. Chau ${ }^{31}$, C.A. Chavez Barajas ${ }^{151}$, S. Che ${ }^{113}$, S. Cheatham ${ }^{167 a, 167 c}$, A. Chegwidden ${ }^{93}$, S. Chekanov ${ }^{6}$, S.V. Chekulaev ${ }^{163 a}$, G.A. Chelkov ${ }^{68, l}$, M.A. Chelstowska ${ }^{32}$, C. Chen ${ }^{36 a}$, C. Chen ${ }^{67}$, H. Chen ${ }^{27}$, J. Chen ${ }^{36 a}$, S. Chen ${ }^{35 b}$, S. Chen ${ }^{157}$, X. Chen ${ }^{35 c, m}$, Y. Chen ${ }^{70}$, H.C. Cheng ${ }^{92}$, H.J. Cheng ${ }^{35 a}$, A. Cheplakov ${ }^{68}$, E. Cheremushkina ${ }^{132}$,

R. Cherkaoui El Moursli137e, E. Cheu ${ }^{7}$, K. Cheung ${ }^{63}$, L. Chevalier ${ }^{138}$, V. Chiarella ${ }^{50,}$ G. Chiarelli126a,126b , G. Chiodini ${ }^{76 a}$, A.S. Chisholm ${ }^{32}$, A. Chitan ${ }^{28 b}$, Y.H. Chiu ${ }^{172}$,

M.V. Chizhov ${ }^{68}$, K. Choi ${ }^{64}$, A.R. Chomont ${ }^{37}$, S. Chouridou ${ }^{156}$, Y.S. Chow ${ }^{62 a}$, V. Christodoulou ${ }^{81}$, M.C. Chu ${ }^{62 a}$, J. Chudoba ${ }^{129}$, A.J. Chuinard ${ }^{90}$, J.J. Chwastowski ${ }^{42}$, L. Chytka ${ }^{117}$, A.K. Ciftci ${ }^{4 a}$,

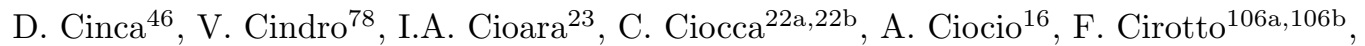
Z.H. Citron ${ }^{175}$, M. Citterio ${ }^{94 a}$, M. Ciubancan ${ }^{28 b}$, A. Clark ${ }^{52}$, B.L. Clark ${ }^{59}$, M.R. Clark ${ }^{38}$, P.J. Clark ${ }^{49}$, R.N. Clarke ${ }^{16}$, C. Clement ${ }^{148 a, 148 b}$, Y. Coadou ${ }^{88}$, M. Cobal ${ }^{167 a, 167 c}$, A. Coccaro ${ }^{52}$, J. Cochran ${ }^{67}$, L. Colasurdo ${ }^{108}$, B. Cole ${ }^{38}$, A.P. Colijn ${ }^{109}$, J. Collot ${ }^{58}$, T. Colombo ${ }^{166}$, P. Conde Muiño ${ }^{128 a, 128 b}$, E. Coniavitis ${ }^{51}$, S.H. Connell ${ }^{147 b}$, I.A. Connelly ${ }^{87}$, S. Constantinescu ${ }^{28 b}$, G. Conti ${ }^{32}$, F. Conventi ${ }^{106 a, n}$, M. Cooke ${ }^{16}$, A.M. Cooper-Sarkar ${ }^{122}$, F. Cormier ${ }^{171}$, K.J.R. Cormier ${ }^{161}$, M. Corradi ${ }^{134 a, 134 b}$, F. Corriveau ${ }^{90, o}$, A. Cortes-Gonzalez ${ }^{32}$, G. Cortiana ${ }^{103}$, 
G. Costa ${ }^{94 a}$, M.J. Costa ${ }^{170}$, D. $\operatorname{Costanzo}^{141}$, G. $\operatorname{Cottin}^{30}$, G. $\operatorname{Cowan}^{80}$, B.E. Cox ${ }^{87}$, K. Cranmer ${ }^{112}$, S.J. Crawley ${ }^{56}$, R.A. Creager ${ }^{124}$, G. Cree ${ }^{31}$, S. Crépé-Renaudin ${ }^{58}$, F. Crescioli ${ }^{83}$, W.A. Cribbs ${ }^{148 a, 148 b}$, M. Cristinziani ${ }^{23}$, V. Croft ${ }^{112}$, G. Crosetti ${ }^{40 a, 40 b}$, A. Cueto ${ }^{85}$, T. Cuhadar Donszelmann ${ }^{141}$, A.R. Cukierman ${ }^{145}$, J. Cummings ${ }^{179}$, M. Curatolo ${ }^{50}$, J. Cúth ${ }^{86}$, S. Czekierda ${ }^{42}$, P. Czodrowski ${ }^{32}$, G. D'amen ${ }^{22 a, 22 b}$, S. D’Auria ${ }^{56}$, L. D'eramo ${ }^{83}$, M. D'Onofrio ${ }^{77}$, M.J. Da Cunha Sargedas De Sousa ${ }^{128 a}, 128 b$, C. Da Via ${ }^{87}$, W. Dabrowski ${ }^{41 a}$, T. Dado ${ }^{146 a}$,

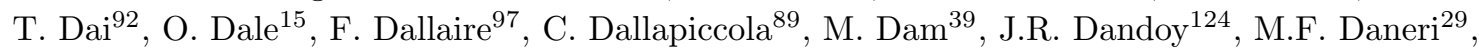
N.P. Dang ${ }^{176}$, A.C. Daniells ${ }^{19}$, N.S. Dann ${ }^{87}$, M. Danninger ${ }^{171}$, M. Dano Hoffmann ${ }^{138}$, V. Dao ${ }^{150}$, G. Darbo ${ }^{53 a}$, S. Darmora ${ }^{8}$, J. Dassoulas ${ }^{3}$, A. Dattagupta ${ }^{118}$, T. Daubney ${ }^{45}$, W. Davey ${ }^{23}$, C. David ${ }^{45}$, T. Davidek ${ }^{131}$, D.R. Davis ${ }^{48}$, P. Davison ${ }^{81}$, E. Dawe ${ }^{91}$, I. Dawson ${ }^{141}$, K. De $^{8}$, R. de Asmundis ${ }^{106 a}$, A. De Benedetti ${ }^{115}$, S. De Castro ${ }^{22 a, 22 b}$, S. De Cecco $^{83}$, N. De Groot ${ }^{108}$, P. de Jong ${ }^{109}$, H. De la Torre ${ }^{93}$, F. De Lorenzi ${ }^{67}$, A. De Maria ${ }^{57}$, D. De Pedis ${ }^{134 a}$, A. De Salvo ${ }^{134 a}$, U. De Sanctis ${ }^{135 a, 135 b}$, A. De Santo ${ }^{151}$, K. De Vasconcelos Corga ${ }^{88}$, J.B. De Vivie De Regie ${ }^{119}$, R. Debbe ${ }^{27}$, C. Debenedetti ${ }^{139}$, D.V. Dedovich ${ }^{68}$, N. Dehghanian ${ }^{3}$, I. Deigaard ${ }^{109}$, M. Del Gaudio ${ }^{40 a, 40 b}$, J. Del Peso ${ }^{85}$, D. Delgove ${ }^{119}$, F. Deliot ${ }^{138}$, C.M. Delitzsch ${ }^{7}$, A. Dell'Acqua ${ }^{32}$, L. Dell'Asta ${ }^{24}$, M. Dell'Orso ${ }^{126 a, 126 b}$, M. Della Pietra ${ }^{106 a, 106 b}$, D. della Volpe ${ }^{52}$, M. Delmastro ${ }^{5}$, C. Delporte ${ }^{119}$, P.A. Delsart ${ }^{58}$, D.A. DeMarco ${ }^{161}$, S. Demers ${ }^{179}$, M. Demichev ${ }^{68}$, A. Demilly ${ }^{83}$, S.P. Denisov ${ }^{132}$, D. Denysiuk ${ }^{138}$, D. Derendarz ${ }^{42}$, J.E. Derkaoui ${ }^{137 d}$, F. Derue ${ }^{83}$,

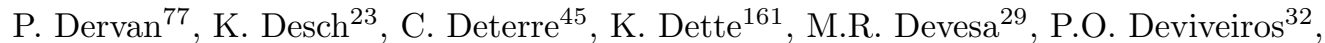
A. Dewhurst ${ }^{133}$, S. Dhaliwal ${ }^{25}$, F.A. Di Bello ${ }^{52}$, A. Di Ciaccio ${ }^{135 a, 135 b}$, L. Di Ciaccio ${ }^{5}$, W.K. Di Clemente ${ }^{124}$, C. Di Donato ${ }^{106 a, 106 b}$, A. Di Girolamo ${ }^{32}$, B. Di Girolamo ${ }^{32}$, B. Di Micco136a,136b, R. Di Nardo ${ }^{32}$, K.F. Di Petrillo ${ }^{59}$, A. Di Simone ${ }^{51}$, R. Di Sipio ${ }^{161}$, D. Di Valentino ${ }^{31}$, C. Diaconu ${ }^{88}$, M. Diamond ${ }^{161}$, F.A. Dias ${ }^{39}$, M.A. Diaz ${ }^{34 a}$, E.B. Diehl ${ }^{92}$, J. Dietrich ${ }^{17}$, S. Díez Cornell ${ }^{45}$, A. Dimitrievska ${ }^{14}$, J. Dingfelder ${ }^{23}$, P. Dita ${ }^{28 b}$, S. Dita ${ }^{28 b}$, F. Dittus ${ }^{32}$, F. Djama ${ }^{88}$, T. Djobava ${ }^{54 b}$, J.I. Djuvsland ${ }^{60 a}$, M.A.B. do Vale ${ }^{26 c}$, D. Dobos ${ }^{32}$, M. Dobre ${ }^{28 b}$, C. Doglioni ${ }^{84}$, J. Dolejsi ${ }^{131}$, Z. Dolezal ${ }^{131}$, M. Donadelli26d , S. Donati126a,126b, P. Dondero ${ }^{123 a, 123 b}$, J. Donini ${ }^{37}$, J. Dopke ${ }^{133}$, A. Doria ${ }^{106 a}$, M.T. Dova ${ }^{74}$, A.T. Doyle ${ }^{56}$, E. Drechsler ${ }^{57}$, M. Dris ${ }^{10}$, Y. Du ${ }^{36 b}$, J. Duarte-Campderros ${ }^{155}$, A. Dubreuil ${ }^{52}$, E. Duchovni ${ }^{175}$, G. Duckeck ${ }^{102}$, A. Ducourthial ${ }^{83}$, O.A. Ducu ${ }^{97, p}$, D. Duda ${ }^{109}$, A. Dudarev ${ }^{32}$, A.Chr. Dudder ${ }^{86}$, E.M. Duffield ${ }^{16}$, L. Duflot ${ }^{119}$, M. Dührssen ${ }^{32}$, M. Dumancic ${ }^{175}$, A.E. Dumitriu ${ }^{28 b}$,

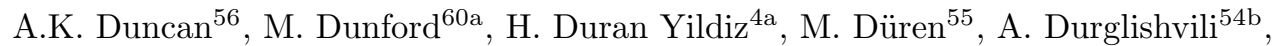
D. Duschinger ${ }^{47}$, B. Dutta ${ }^{45}$, D. Duvnjak ${ }^{1}$, M. Dyndal ${ }^{45}$, B.S. Dziedzic ${ }^{42}$, C. Eckardt ${ }^{45}$, K.M. Ecker ${ }^{103}$, R.C. Edgar ${ }^{92}$, T. Eifert ${ }^{32}$, G. Eigen ${ }^{15}$, K. Einsweiler ${ }^{16}$, T. Ekelof ${ }^{168}$, M. El Kacimi ${ }^{137 c}$, R. El Kosseifi ${ }^{88}$, V. Ellajosyula ${ }^{88}$, M. Ellert ${ }^{168}$, S. Elles ${ }^{5}$, F. Ellinghaus ${ }^{178}$, A.A. Elliot ${ }^{172}$, N. Ellis ${ }^{32}$, J. Elmsheuser ${ }^{27}$, M. Elsing ${ }^{32}$, D. Emeliyanov ${ }^{133}$, Y. Enari ${ }^{157}$, O.C. Endner ${ }^{86}$, J.S. Ennis ${ }^{173}$, J. Erdmann ${ }^{46}$, A. Ereditato ${ }^{18}$, M. Ernst ${ }^{27}$, S. Errede ${ }^{169}$, M. Escalier ${ }^{119}$, C. Escobar ${ }^{170}$, B. Esposito ${ }^{50}$, O. Estrada Pastor ${ }^{170}$, A.I. Etienvre ${ }^{138}$, E. Etzion ${ }^{155}$, H. Evans ${ }^{64}$, A. Ezhilov ${ }^{125}$, M. Ezzi ${ }^{137 e}$, F. Fabbri ${ }^{22 a, 22 b}$, L. Fabbri ${ }^{22 a, 22 b}$, V. Fabiani ${ }^{108,}$ G. Facini ${ }^{81}$, R.M. Fakhrutdinov ${ }^{132}$, S. Falciano ${ }^{134 a}$, R.J. Falla ${ }^{81}$, J. Faltova ${ }^{32}$, Y. Fang ${ }^{35 a}$, M. Fanti ${ }^{94 a, 94 b}$, A. Farbin ${ }^{8}$, A. Farilla ${ }^{136 a}$, C. Farina ${ }^{127}$, E.M. Farina ${ }^{123 a, 123 b}$, T. Farooque ${ }^{93}$, S. Farrell ${ }^{16}$, S.M. Farrington ${ }^{173}$, P. Farthouat ${ }^{32}$, F. Fassi ${ }^{137 e}$, P. Fassnacht ${ }^{32}$, D. Fassouliotis ${ }^{9}$, M. Faucci Giannelli ${ }^{49}$, A. Favareto $53 \mathrm{a}, 53 \mathrm{~b}$, W.J. Fawcett ${ }^{122}$, L. Fayard ${ }^{119}$, O.L. Fedin ${ }^{125, q}$, W. Fedorko ${ }^{171}$, S. Feigl ${ }^{121}$, L. Feligioni ${ }^{88}$, C. Feng ${ }^{36 b}$, E.J. Feng ${ }^{32}$, H. Feng ${ }^{92}$, M.J. Fenton ${ }^{56}$, A.B. Fenyuk ${ }^{132}$, L. Feremenga ${ }^{8}$, P. Fernandez Martinez ${ }^{170}$, S. Fernandez Perez ${ }^{13}$, J. Ferrando ${ }^{45}$, A. Ferrari ${ }^{168}$, P. Ferrari ${ }^{109}$, R. Ferrari ${ }^{123 a}$, D.E. Ferreira de Lima $^{60 b}$, A. Ferrer ${ }^{170}$, D. Ferrere ${ }^{52}$, C. Ferretti ${ }^{92}$, F. Fiedler ${ }^{86}$, A. Filipčič ${ }^{78}$, M. Filipuzzi ${ }^{45}$, F. Filthaut ${ }^{108}$, M. Fincke-Keeler ${ }^{172}$, K.D. Finelli ${ }^{152}$, M.C.N. Fiolhais ${ }^{128 a, 128 c, r}$, L. Fiorini ${ }^{170}$, A. Fischer ${ }^{2}$, C. Fischer ${ }^{13}$, J. Fischer ${ }^{178}$, W.C. Fisher ${ }^{93}$, N. Flaschel ${ }^{45}$, I. Fleck ${ }^{143}$, P. Fleischmann ${ }^{92}$, R.R.M. Fletcher ${ }^{124}$, T. Flick ${ }^{178}$, 
B.M. Flierl ${ }^{102}$, L.R. Flores Castillo ${ }^{62 a}$, M.J. Flowerdew ${ }^{103}$, G.T. Forcolin ${ }^{87}$, A. Formica ${ }^{138}$, F.A. Förster ${ }^{13}$, A. Forti ${ }^{87}$, A.G. Foster ${ }^{19}$, D. Fournier ${ }^{119}$, H. Fox ${ }^{75}$, S. Fracchia ${ }^{141}$, P. Francavilla ${ }^{83}$, M. Franchini ${ }^{22 a, 22 b}$, S. Franchino ${ }^{60 a}$, D. Francis $^{32}$, L. Franconi ${ }^{121}$, M. Franklin ${ }^{59}$, M. Frate ${ }^{166}$, M. Fraternali ${ }^{123 a, 123 b}$, D. Freeborn ${ }^{81}$, S.M. Fressard-Batraneanu ${ }^{32}$, B. Freund ${ }^{97}$, D. Froidevaux ${ }^{32}$, J.A. Frost ${ }^{122}$, C. Fukunaga ${ }^{158}$, T. Fusayasu ${ }^{104}$, J. Fuster ${ }^{170}$, C. Gabaldon ${ }^{58}$, O. Gabizon ${ }^{154}$, A. Gabriellii2a,22b, A. Gabrielli ${ }^{16}$, G.P. Gach ${ }^{41 a}$, S. Gadatsch ${ }^{32}$, S. Gadomski ${ }^{80}$, G. Gagliardi ${ }^{53 a, 53 b}$, L.G. Gagnon ${ }^{97}$, C. Galea ${ }^{108}$, B. Galhardo ${ }^{128 a, 128 c}$, E.J. Gallas ${ }^{122}$, B.J. Gallop ${ }^{133}$, P. Gallus ${ }^{130}$, G. Galster ${ }^{39}$, K.K. Gan ${ }^{113}$, S. Ganguly ${ }^{37}$, Y. Gao ${ }^{77}$, Y.S. Gao ${ }^{145, g}$, F.M. Garay Walls ${ }^{34 a}$, C. García ${ }^{170}$, J.E. García Navarro ${ }^{170}$, J.A. García Pascual ${ }^{35 a}$, M. Garcia-Sciveres ${ }^{16}$, R.W. Gardner ${ }^{33}$, N. Garelli ${ }^{145}$, V. Garonne ${ }^{121}$, A. Gascon Bravo ${ }^{45}$, K. Gasnikova ${ }^{45}$, C. Gatti ${ }^{50}$, A. Gaudiello ${ }^{53 a, 53 b}$, G. Gaudio ${ }^{123 a}$, I.L. Gavrilenko ${ }^{98}$, C. Gay ${ }^{171}$, G. Gaycken ${ }^{23}$, E.N. Gazis ${ }^{10}$, C.N.P. Gee ${ }^{133}$, J. Geisen ${ }^{57}$, M. Geisen ${ }^{86}$, M.P. Geisler ${ }^{60 a}$, K. Gellerstedt ${ }^{148 a, 148 b}$, C. Gemme ${ }^{53 a}$, M.H. Genest ${ }^{58}$, C. Geng ${ }^{92}$, S. Gentile ${ }^{134 a, 134 b}$, C. Gentsos ${ }^{156}$, S. George ${ }^{80}$, D. Gerbaudo ${ }^{13}$, A. Gershon ${ }^{155}$, G. Geßner ${ }^{46}$, S. Ghasemi ${ }^{143}$, M. Ghneimat ${ }^{23}$, B. Giacobbe ${ }^{22 a}$, S. Giagu ${ }^{134 a, 134 b}$, N. Giangiacomi ${ }^{22 a, 22 b}$, P. Giannetti1 ${ }^{126 a, 126 b}$, S.M. Gibson ${ }^{80}$, M. Gignac ${ }^{171}$, M. Gilchriese ${ }^{16}$, D. Gillberg ${ }^{31}$, G. Gilles ${ }^{178}$, D.M. Gingrich ${ }^{3, d}$, M.P. Giordani ${ }^{167 a, 167 c}$, F.M. Giorgi ${ }^{22 a}$, P.F. Giraud ${ }^{138}$, P. Giromini ${ }^{59}$, G. Giugliarelli ${ }^{167 a, 167 c}$, D. Giugni ${ }^{94 a}$, F. Giuli ${ }^{122}$, C. Giuliani ${ }^{103}$, M. Giulini ${ }^{60 b}$, B.K. Gjelsten ${ }^{121}$, S. Gkaitatzis ${ }^{156}$, I. Gkialas ${ }^{9, s}$, E.L. Gkougkousis ${ }^{13}$, P. Gkountoumis ${ }^{10}$, L.K. Gladilin ${ }^{101}$, C. Glasman ${ }^{85}$, J. Glatzer ${ }^{13}$, P.C.F. Glaysher ${ }^{45}$, A. Glazov ${ }^{45}$, M. Goblirsch-Kolb ${ }^{25}$, J. Godlewski ${ }^{42}$, S. Goldfarb ${ }^{91}$, T. Golling ${ }^{52}$, D. Golubkov ${ }^{132}$, A. Gomes ${ }^{128 a, 128 b, 128 d}$, R. Gonçalo ${ }^{128 a}$, R. Goncalves Gama ${ }^{26 a}$, J. Goncalves Pinto Firmino Da Costa ${ }^{138}$, G. Gonella ${ }^{51}$, L. Gonella $^{19}$, A. Gongadze ${ }^{68}$, S. González de la Hoz ${ }^{170}$, S. Gonzalez-Sevilla ${ }^{52}$, L. Goossens ${ }^{32}$, P.A. Gorbounov ${ }^{99}$, H.A. Gordon ${ }^{27}$, I. Gorelov ${ }^{107}$, B. Gorini ${ }^{32}$, E. Gorini ${ }^{76 a, 76 b}$, A. Gorišek ${ }^{78}$, A.T. Goshaw ${ }^{48}$, C. Gössling ${ }^{46}$, M.I. Gostkin ${ }^{68}$, C.A. Gottardo ${ }^{23}$, C.R. Goudet ${ }^{119}$, D. Goujdami ${ }^{137 c}$, A.G. Goussiou ${ }^{140}$, N. Govender ${ }^{147 b, t}$, E. Gozani ${ }^{154}$, L. Graber ${ }^{57}$, I. Grabowska-Bold ${ }^{41 a}$, P.O.J. Gradin ${ }^{168}$, J. Gramling ${ }^{166}$, E. Gramstad ${ }^{121}$, S. Grancagnolo ${ }^{17}$, V. Gratchev ${ }^{125}$, P.M. Gravila ${ }^{28 f}$, C. Gray ${ }^{56}$, H.M. Gray ${ }^{16}$, Z.D. Greenwood ${ }^{82, u}$, C. Grefe $^{23}$, K. Gregersen ${ }^{81}$, I.M. Gregor ${ }^{45}$, P. Grenier ${ }^{145}$, K. Grevtsov ${ }^{5}$, J. Griffiths ${ }^{8}$, A.A. Grillo ${ }^{139}$, K. Grimm ${ }^{75}$, S. Grinstein ${ }^{13, v}$, Ph. Gris ${ }^{37}$, J.-F. Grivaz ${ }^{119}$, S. Groh ${ }^{86}$, E. Gross ${ }^{175}$, J. Grosse-Knetter ${ }^{57}$, G.C. Grossi ${ }^{82}$, Z.J. Grout ${ }^{81}$, A. Grummer ${ }^{107}$, L. Guan ${ }^{92}$, W. Guan ${ }^{176}$, J. Guenther ${ }^{65}$, F. Guescini ${ }^{163 a}$, D. Guest ${ }^{166}$, O. Gueta ${ }^{155}$, B. Guil ${ }^{113}$, E. Guido ${ }^{53 a, 53 b}$, T. Guillemin ${ }^{5}$, S. Guindon ${ }^{32}$, U. Gul ${ }^{56}$, C. Gumpert ${ }^{32}$,

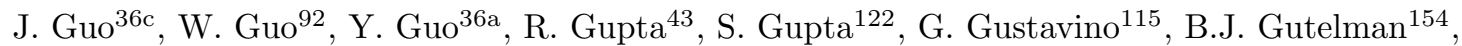
P. Gutierrez ${ }^{115}$, N.G. Gutierrez Ortiz ${ }^{81}$, C. Gutschow ${ }^{81}$, C. Guyot ${ }^{138}$, M.P. Guzik ${ }^{41 a}$, C. Gwenlan ${ }^{122}$, C.B. Gwilliam ${ }^{77}$, A. Haas ${ }^{112}$, C. Haber ${ }^{16}$, H.K. Hadavand ${ }^{8}$, N. Haddad ${ }^{137 e}$, A. Hadef ${ }^{88}$, S. Hageböck ${ }^{23}$, M. Hagihara ${ }^{164}$, H. Hakobyan ${ }^{180, *}$, M. Haleem ${ }^{45}$, J. Haley ${ }^{116}$,

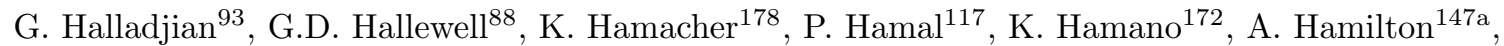
G.N. Hamity ${ }^{141}$, P.G. Hamnett ${ }^{45}$, L. Han ${ }^{36 a}$, S. $\operatorname{Han}^{35 a}$, K. Hanagaki ${ }^{69, w}$, K. Hanawa ${ }^{157}$, M. Hance ${ }^{139}$, B. Haney ${ }^{124}$, P. Hanke ${ }^{60 a}$, J.B. Hansen ${ }^{39}$, J.D. Hansen ${ }^{39}$, M.C. Hansen ${ }^{23}$,

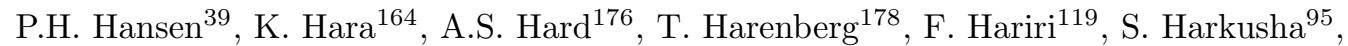
P.F. Harrison ${ }^{173}$, N.M. Hartmann ${ }^{102}$, Y. Hasegawa ${ }^{142}$, A. Hasib ${ }^{49}$, S. Hassani ${ }^{138}$, S. Haug ${ }^{18}$, R. Hauser ${ }^{93}$, L. Hauswald ${ }^{47}$, L.B. Havener ${ }^{38}$, M. Havranek ${ }^{130}$, C.M. Hawkes ${ }^{19}$, R.J. Hawkings ${ }^{32}$, D. Hayakawa ${ }^{159}$, D. Hayden ${ }^{93}$, C.P. Hays ${ }^{122}$, J.M. Hays ${ }^{79}$, H.S. Hayward ${ }^{77}$, S.J. Haywood ${ }^{133}$,

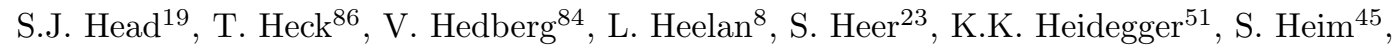
T. Heim ${ }^{16}$, B. Heinemann ${ }^{45, x}$, J.J. Heinrich ${ }^{102}$, L. Heinrich ${ }^{112}$, C. Heinz ${ }^{55}$, J. Hejbal ${ }^{129}$, L. Helary ${ }^{32}$, A. Held ${ }^{171}$, S. Hellman ${ }^{148 a, 148 b}$, C. Helsens ${ }^{32}$, R.C.W. Henderson ${ }^{75}$, Y. Heng ${ }^{176}$, S. Henkelmann ${ }^{171}$, A.M. Henriques Correia ${ }^{32}$, S. Henrot-Versille ${ }^{119}$, G.H. Herbert ${ }^{17}$, H. Herde ${ }^{25}$, V. Herget ${ }^{177}$, Y. Hernández Jiménez ${ }^{147 c}$, H. Herr ${ }^{86}$, G. Herten ${ }^{51}$, R. Hertenberger ${ }^{102}$, L. Hervas ${ }^{32}$, 
T.C. Herwig ${ }^{124}$, G.G. Hesketh ${ }^{81}$, N.P. Hessey ${ }^{163 a}$, J.W. Hetherly ${ }^{43}$, S. Higashino ${ }^{69}$, E. Higón-Rodriguez ${ }^{170}$, K. Hildebrand ${ }^{33}$, E. Hill ${ }^{172}$, J.C. Hill ${ }^{30}$, K.H. Hiller ${ }^{45}$, S.J. Hillier ${ }^{19}$, M. Hils ${ }^{47}$, I. Hinchliffe ${ }^{16}$, M. Hirose ${ }^{51}$, D. Hirschbuehl ${ }^{178}$, B. Hiti ${ }^{78}$, O. Hladik ${ }^{129}$, X. Hoad ${ }^{49}$, J. Hobbs ${ }^{150}$, N. Hod ${ }^{163 a}$, M.C. Hodgkinson ${ }^{141}$, P. Hodgson ${ }^{141}$, A. Hoecker ${ }^{32}$, M.R. Hoeferkamp ${ }^{107}$, F. Hoenig ${ }^{102}$, D. Hohn ${ }^{23}$, T.R. Holmes ${ }^{33}$, M. Homann ${ }^{46}$, S. Honda ${ }^{164}$, T. Honda ${ }^{69}$, T.M. Hong ${ }^{127}$, B.H. Hooberman ${ }^{169}$, W.H. Hopkins ${ }^{118}$, Y. Horii ${ }^{105}$, A.J. Horton ${ }^{144}$, J-Y. Hostachy ${ }^{58}$, S. Hou ${ }^{153}$,

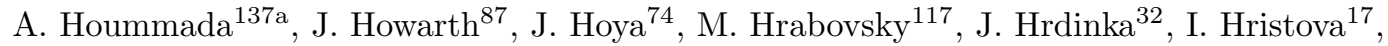
J. Hrivnac ${ }^{119}$, T. Hryn'ova ${ }^{5}$, A. Hrynevich ${ }^{96}$, P.J. Hsu ${ }^{63}$, S.-C. Hsu ${ }^{140}$, Q. Hu ${ }^{36 a}$, S. Hu ${ }^{36 c}$, Y. Huang ${ }^{35 a}$, Z. Hubacek ${ }^{130}$, F. Hubaut ${ }^{88}$, F. Huegging ${ }^{23}$, T.B. Huffman ${ }^{122}$, E.W. Hughes ${ }^{38}$, G. Hughes ${ }^{75}$, M. Huhtinen ${ }^{32}$, P. Huo ${ }^{150}$, N. Huseynov ${ }^{68, b}$, J. Huston ${ }^{93}$, J. Huth ${ }^{59}$, G. Iacobucci ${ }^{52}$, G. Iakovidis $^{27}$, I. Ibragimov ${ }^{143}$, L. Iconomidou-Fayard ${ }^{119}$, Z. Idrissi ${ }^{137 e}$, P. Iengo $^{32}$,

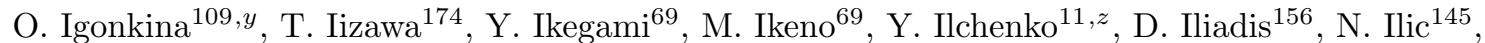

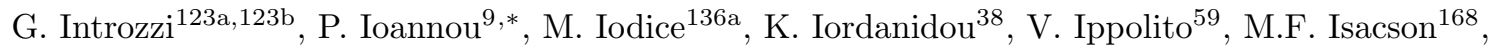

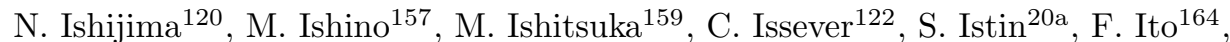
J.M. Iturbe Ponce ${ }^{62 a}$, R. Iuppa ${ }^{162 a, 162 b}$, H. Iwasaki ${ }^{69}$, J.M. Izen ${ }^{44}$, V. Izzo ${ }^{106 a}$, S. Jabbar ${ }^{3}$, P. Jackson ${ }^{1}$, R.M. Jacobs ${ }^{23}$, V. Jain ${ }^{2}$, K.B. Jakobi ${ }^{86}$, K. Jakobs ${ }^{51}$, S. Jakobsen ${ }^{65}$, T. Jakoubek ${ }^{129}$, D.O. Jamin ${ }^{116}$, D.K. Jana ${ }^{82}$, R. Jansky ${ }^{52}$, J. Janssen ${ }^{23}$, M. Janus ${ }^{57}$, P.A. Janus ${ }^{41 a}$, G. Jarlskog ${ }^{84}$, N. Javadov ${ }^{68, b}$, T. Javůrek ${ }^{51}$, M. Javurkova ${ }^{51}$, F. Jeanneau ${ }^{138}$, L. Jeanty ${ }^{16}$, J. Jejelava ${ }^{54 a, a a}$, A. Jelinskas ${ }^{173}$, P. Jenni ${ }^{51, a b}$, C. Jeske ${ }^{173}$, S. Jézéquel ${ }^{5}$, H. Ji ${ }^{176}$, J. Jia ${ }^{150}$, H. Jiang ${ }^{67}$, Y. Jiang ${ }^{36 a}$, Z. Jiang ${ }^{145}$, S. Jiggins ${ }^{81}$, J. Jimenez Pena ${ }^{170}$, S. Jin ${ }^{35 a}$, A. Jinaru ${ }^{28 b}$, O. Jinnouchi ${ }^{159}$, H. Jivan ${ }^{147 c}$, P. Johansson ${ }^{141}$, K.A. Johns ${ }^{7}$, C.A. Johnson ${ }^{64}$, W.J. Johnson ${ }^{140}$, K. Jon-And ${ }^{148 a, 148 b}$, R.W.L. Jones ${ }^{75}$, S.D. Jones ${ }^{151}$, S. Jones ${ }^{7}$, T.J. Jones ${ }^{77}$, J. Jongmanns ${ }^{60 a}$, P.M. Jorge ${ }^{128 a, 128 b}$, J. Jovicevic ${ }^{163 a}$, X. Ju ${ }^{176}$, A. Juste Rozas ${ }^{13, v}$, M.K. Köhler ${ }^{175}$,

A. Kaczmarska ${ }^{42}$, M. Kado ${ }^{119}$, H. Kagan ${ }^{113}$, M. Kagan ${ }^{145}$, S.J. Kahn ${ }^{88}$, T. Kaji ${ }^{174}$, E. Kajomovitz ${ }^{48}$, C.W. Kalderon ${ }^{84}$, A. Kaluza ${ }^{86}$, S. Kama ${ }^{43}$, A. Kamenshchikov ${ }^{132}$, N. Kanaya ${ }^{157}$, L. Kanjir ${ }^{78}$, V.A. Kantserov ${ }^{100}$, J. Kanzaki ${ }^{69}$, B. Kaplan ${ }^{112}$, L.S. Kaplan ${ }^{176}$, D. $\operatorname{Kar}^{147 c}$, K. Karakostas ${ }^{10}$, N. Karastathis ${ }^{10}$, M.J. Kareem ${ }^{57}$, E. Karentzos ${ }^{10}$, S.N. Karpov ${ }^{68}$, Z.M. Karpova ${ }^{68}$, K. Karthik ${ }^{112}$, V. Kartvelishvili ${ }^{75}$, A.N. Karyukhin ${ }^{132}$, K. Kasahara ${ }^{164}$, L. Kashif ${ }^{176}$, R.D. Kass ${ }^{113}$, A. Kastanas ${ }^{149}$, Y. Kataoka ${ }^{157}$, C. Kato ${ }^{157}$, A. Katre ${ }^{52}$, J. Katzy ${ }^{45}$, K. Kawade ${ }^{70}$, K. Kawagoe ${ }^{73}$, T. Kawamoto ${ }^{157}$, G. Kawamura ${ }^{57}$, E.F. Kay ${ }^{77}$, V.F. Kazanin ${ }^{111, c}$, R. Keeler ${ }^{172}$, R. Kehoe ${ }^{43}$, J.S. Keller ${ }^{31}$, E. Kellermann ${ }^{84}$, J.J. Kempster ${ }^{80}$, J Kendrick ${ }^{19}$, H. Keoshkerian ${ }^{161}$, O. Kepka ${ }^{129}$, B.P. Kerševan ${ }^{78}$, S. Kersten ${ }^{178}$, R.A. Keyes ${ }^{90}$, M. Khader ${ }^{169}$, F. Khalil-zada ${ }^{12}$, A. Khanov ${ }^{116}$, A.G. Kharlamov ${ }^{111, c}$, T. Kharlamova ${ }^{111, c}$, A. Khodinov ${ }^{160}$, T.J. Khoo ${ }^{52}$, V. Khovanskiy ${ }^{99, *}$, E. Khramov ${ }^{68}$, J. Khubua ${ }^{54 b, a c}$, S. Kido ${ }^{70}$, C.R. Kilby ${ }^{80}$, H.Y. Kim ${ }^{8}$, S.H. Kim ${ }^{164}$, Y.K. Kim ${ }^{33}$, N. Kimura ${ }^{156}$, O.M. Kind ${ }^{17}$, B.T. King ${ }^{77}$, D. Kirchmeier ${ }^{47}$, J. Kirk ${ }^{133}$, A.E. Kiryunin ${ }^{103}$, T. Kishimoto ${ }^{157}$, D. Kisielewska ${ }^{41 a}$, V. Kitali ${ }^{45}$, O. Kivernyk ${ }^{5}$, E. Kladiva ${ }^{146 b}$, T. Klapdor-Kleingrothaus ${ }^{51}$, M.H. Klein ${ }^{92}$, M. Klein ${ }^{77}$, U. Klein ${ }^{77}$, K. Kleinknecht ${ }^{86}$, P. Klimek ${ }^{110}$, A. Klimentov ${ }^{27}$, R. Klingenberg ${ }^{46}$, T. Klingl ${ }^{23}$, T. Klioutchnikova ${ }^{32}$, E.-E. Kluge ${ }^{60 a}$, P. Kluit ${ }^{109}$, S. Kluth ${ }^{103}$, E. Kneringer ${ }^{65}$, E.B.F.G. Knoops ${ }^{88}$, A. Knue ${ }^{103}$, A. Kobayashi ${ }^{157}$, D. Kobayashi ${ }^{159}$, T. Kobayashi ${ }^{157}$, M. Kobel ${ }^{47}$, M. Kocian ${ }^{145}$, P. Kodys ${ }^{131}$, T. Koffas ${ }^{31}$, E. Koffeman ${ }^{109}$, N.M. Köhler ${ }^{103}$, T. Koi ${ }^{145}$, M. Kolb ${ }^{60 b}$, I. Koletsou ${ }^{5}$,

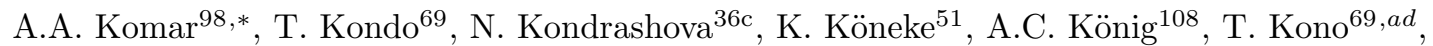
R. Konoplich ${ }^{112, a e}$, N. Konstantinidis ${ }^{81}$, R. Kopeliansky ${ }^{64}$, S. Koperny ${ }^{41 a}$, A.K. Kopp ${ }^{51}$, K. Korcyl ${ }^{42}$, K. Kordas ${ }^{156}$, A. Korn ${ }^{81}$, A.A. Korol ${ }^{111, c}$, I. Korolkov ${ }^{13}$, E.V. Korolkova ${ }^{141}$, O. Kortner ${ }^{103}$, S. Kortner ${ }^{103}$, T. Kosek ${ }^{131}$, V.V. Kostyukhin ${ }^{23}$, A. Kotwal ${ }^{48}$, A. Koulouris ${ }^{10}$, A. Kourkoumeli-Charalampidi ${ }^{123 a, 123 b}$, C. Kourkoumelis ${ }^{9}$, E. Kourlitis ${ }^{141}$, V. Kouskoura ${ }^{27}$, A.B. Kowalewska ${ }^{42}$, R. Kowalewski ${ }^{172}$, T.Z. Kowalski ${ }^{41 a}$, C. Kozakai ${ }^{157}$, W. Kozanecki ${ }^{138}$, A.S. Kozhin ${ }^{132}$, V.A. Kramarenko ${ }^{101}$, G. Kramberger ${ }^{78}$, D. Krasnopevtsev ${ }^{100}$, M.W. Krasny ${ }^{83}$, 
A. Krasznahorkay ${ }^{32}$, D. Krauss ${ }^{103}$, J.A. Kremer ${ }^{41 a}$, J. Kretzschmar ${ }^{77}$, K. Kreutzfeldt ${ }^{55}$, P. Krieger ${ }^{161}$, K. Krizka ${ }^{16}$, K. Kroeninger ${ }^{46}$, H. Kroha ${ }^{103}$, J. Kroll ${ }^{129}$, J. Kroll ${ }^{124}$, J. Kroseberg ${ }^{23}$, J. Krstic ${ }^{14}$, U. Kruchonak ${ }^{68}$, H. Krüger ${ }^{23}$, N. Krumnack ${ }^{67}$, M.C. Kruse ${ }^{48}$, T. Kubota ${ }^{91}$, H. Kucuk ${ }^{81}$, S. Kuday ${ }^{4 b}$, J.T. Kuechler ${ }^{178}$, S. Kuehn ${ }^{32}$, A. Kugel ${ }^{60 a}$, F. Kuger ${ }^{177}$, T. Kuhl ${ }^{45}$, V. Kukhtin ${ }^{68}$, R. Kukla ${ }^{88}$, Y. Kulchitsky ${ }^{95}$, S. Kuleshov ${ }^{34 b}$, Y.P. Kulinich ${ }^{169}$, M. Kuna ${ }^{134 a, 134 b}$, T. Kunigo ${ }^{71}$, A. Kupco ${ }^{129}$, T. Kupfer ${ }^{46}$, O. Kuprash ${ }^{155}$, H. Kurashige ${ }^{70}$, L.L. Kurchaninov ${ }^{163 a}$, Y.A. Kurochkin ${ }^{95}$, M.G. Kurth ${ }^{35 a}$, V. Kus ${ }^{129}$, E.S. Kuwertz ${ }^{172}$, M. Kuze ${ }^{159}$, J. Kvita ${ }^{117}$, T. Kwan ${ }^{172}$, D. Kyriazopoulos ${ }^{141}$, A. La Rosa ${ }^{103}$, J.L. La Rosa Navarro ${ }^{26 \mathrm{~d}}$, L. La Rotonda ${ }^{40 a}$,40b, F. La Ruffa ${ }^{40 a, 40 b}$, C. Lacasta ${ }^{170}$, F. Lacava ${ }^{134 a, 134 b}$, J. Lacey ${ }^{45}$, D.P.J. Lack ${ }^{87}$, H. Lacker ${ }^{17}$,

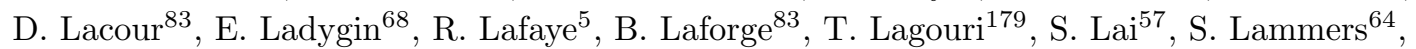
W. Lampl ${ }^{7}$, E. Lançon ${ }^{27}$, U. Landgraf ${ }^{51}$, M.P.J. Landon ${ }^{79}$, M.C. Lanfermann ${ }^{52}$, V.S. Lang ${ }^{45}$, J.C. Lange ${ }^{13}$, R.J. Langenberg ${ }^{32}$, A.J. Lankford ${ }^{166}$, F. Lanni ${ }^{27}$, K. Lantzsch ${ }^{23}$, A. Lanza ${ }^{123 a}$, A. Lapertosa ${ }^{53 a, 53 b}$, S. Laplace ${ }^{83}$, J.F. Laporte ${ }^{138}$, T. Lari ${ }^{94 a}$, F. Lasagni Manghi22a,22b,

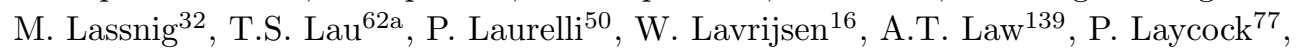
T. Lazovich ${ }^{59}$, M. Lazzaroni ${ }^{94 a}$,94b, B. Le ${ }^{91}$, O. Le Dortz ${ }^{83}$, E. Le Guirriec ${ }^{88}$, E.P. Le Quilleuc ${ }^{138}$, M. LeBlanc ${ }^{172}$, T. LeCompte ${ }^{6}$, F. Ledroit-Guillon ${ }^{58}$, C.A. Lee ${ }^{27}$, G.R. Lee ${ }^{133, a f}$, S.C. Lee ${ }^{153}$, L. Lee ${ }^{59}$, B. Lefebvre ${ }^{90}$, G. Lefebvre ${ }^{83}$, M. Lefebvre ${ }^{172}$, F. Legger ${ }^{102}$, C. Leggett ${ }^{16}$, G. Lehmann Miotto ${ }^{32}$, X. Lei ${ }^{7}$, W.A. Leightt ${ }^{45}$, M.A.L. Leite ${ }^{26 \mathrm{~d}}$, R. Leitner ${ }^{131}$, D. Lellouch ${ }^{175}$, B. Lemmer ${ }^{57}$, K.J.C. Leney ${ }^{81}$, T. Lenz ${ }^{23}$, B. Lenzi ${ }^{32}$, R. Leone ${ }^{7}$, S. Leone ${ }^{126 a, 126 b}$, C. Leonidopoulos ${ }^{49}$, G. Lerner ${ }^{151}$, C. Leroy ${ }^{97}$, A.A.J. Lesage ${ }^{138}$, C.G. Lester ${ }^{30}$, M. Levchenko ${ }^{125}$, J. Levêque ${ }^{5}$, D. Levin ${ }^{92}$, L.J. Levinson ${ }^{175}$, M. Levy ${ }^{19}$, D. Lewis ${ }^{79}$, B. $\mathrm{Li}^{36 a, a g}$, Changqiao $\mathrm{Li}^{36 a}$,

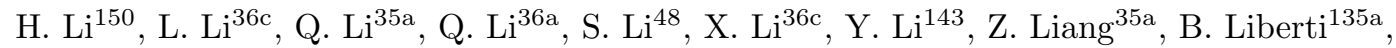
A. Liblong ${ }^{161}$, K. Lie ${ }^{62 c}$, J. Liebal ${ }^{23}$, W. Liebig ${ }^{15}$, A. Limosani ${ }^{152}$, S.C. $\operatorname{Lin}^{182}$, T.H. Lin ${ }^{86}$, R.A. Linck ${ }^{64}$, B.E. Lindquist ${ }^{150}$, A.E. Lionti ${ }^{52}$, E. Lipeles ${ }^{124}$, A. Lipniacka ${ }^{15}$, M. Lisovyi ${ }^{60 b}$, T.M. Liss ${ }^{169, a h}$, A. Lister ${ }^{171}$, A.M. Litke ${ }^{139}$, B. Liu ${ }^{67}$, H. Liu ${ }^{92}$, H. Liu ${ }^{27}$, J.K.K. Liu ${ }^{122}$, J. Liu ${ }^{36 b}$, J.B. Liu ${ }^{36 a}$, K. Liu ${ }^{88}$, L. Liu ${ }^{169}$, M. Liu ${ }^{36 a}$, Y.L. Liu ${ }^{36 a}$, Y. Liu ${ }^{36 a}$, M. Livan ${ }^{123 a, 123 b}$, A. Lleres ${ }^{58}$, J. Llorente Merino ${ }^{35 a}$, S.L. Lloyd ${ }^{79}$, C.Y. Lo ${ }^{62 b}$, F. Lo Sterzo ${ }^{153}$, E.M. Lobodzinska ${ }^{45}$, P. Loch ${ }^{7}$, F.K. Loebinger ${ }^{87}$, A. Loesle ${ }^{51}$, K.M. Loew ${ }^{25}$, A. Loginov $^{179, *}$, T. Lohse ${ }^{17}$, K. Lohwasser ${ }^{141}$, M. Lokajicek ${ }^{129}$, B.A. Long ${ }^{24}$, J.D. Long ${ }^{169}$, R.E. Long ${ }^{75}$, L. Longo $76 a, 76 b$, K.A. Looper ${ }^{113}$, J.A. Lopez ${ }^{34 b}$, D. Lopez Mateos ${ }^{59}$, I. Lopez Paz ${ }^{13}$, A. Lopez Solis ${ }^{83}$, J. Lorenz ${ }^{102}$,

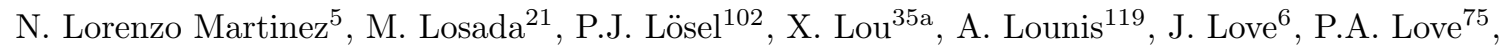
H. Lu ${ }^{62 a}$, N. $\mathrm{Lu}^{92}$, Y.J. Lu ${ }^{63}$, H.J. Lubatti1 ${ }^{140}$, C. Luci ${ }^{134 a, 134 b}$, A. Lucotte ${ }^{58}$, C. Luedtke ${ }^{51}$, F. Luehring ${ }^{64}$, W. Lukas $^{65}$, L. Luminari ${ }^{134 a}$, O. Lundberg ${ }^{148 a, 148 b}$, B. Lund-Jensen ${ }^{149}$,

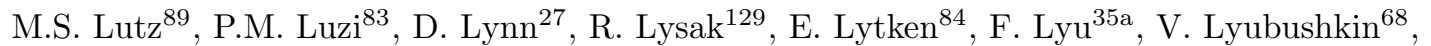
H. $\mathrm{Ma}^{27}$, L.L. $\mathrm{Ma}^{36 \mathrm{~b}}$, Y. $\mathrm{Ma}^{36 \mathrm{~b}}$, G. Maccarrone ${ }^{50}$, A. Macchiolo ${ }^{103}$, C.M. Macdonald ${ }^{141}$, B. Maček ${ }^{78}$, J. Machado Miguens ${ }^{124,128 b}$, D. Madaffari ${ }^{170}$, R. Madar ${ }^{37}$, W.F. Mader ${ }^{47}$, A. Madsen ${ }^{45}$, J. Maeda ${ }^{70}$, S. Maeland ${ }^{15}$, T. Maeno ${ }^{27}$, A.S. Maevskiy ${ }^{101}$, V. Magerl ${ }^{51}$, J. Mahlstedt ${ }^{109}$, C. Maiani ${ }^{119}$, C. Maidantchik ${ }^{26 a}$, A.A. Maier ${ }^{103}$, T. Maier ${ }^{102}$, A. Maio ${ }^{128 a, 128 b, 128 d}$, O. Majersky ${ }^{146 a}$, S. Majewski ${ }^{118}$, Y. Makida $^{69}$, N. Makovec ${ }^{119}$, B. Malaescu ${ }^{83}$, Pa. Malecki ${ }^{42}$, V.P. Maleev ${ }^{125}$, F. Malek ${ }^{58}$, U. Mallik ${ }^{66}$, D. Malon ${ }^{6}$, C. Malone ${ }^{30}$, S. Maltezos ${ }^{10}$, S. Malyukov ${ }^{32}$, J. Mamuzic ${ }^{170}$, G. Mancini ${ }^{50}$, I. Mandić ${ }^{78}$, J. Maneira ${ }^{128 a, 128 b}$, L. Manhaes de Andrade Filho ${ }^{26 b}$, J. Manjarres Ramos ${ }^{47}$, K.H. Mankinen ${ }^{84}$, A. Mann ${ }^{102}$, A. Manousos ${ }^{32}$, B. Mansoulie ${ }^{138}$, J.D. Mansour ${ }^{35 a}$, R. Mantifel ${ }^{90}$, M. Mantoani ${ }^{57}$,

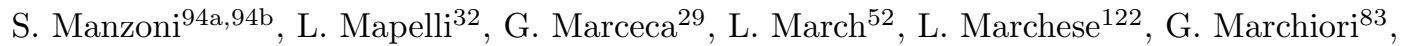
M. Marcisovsky ${ }^{129}$, C.A. Marin Tobon $^{32}$, M. Marjanovic ${ }^{37}$, D.E. Marley ${ }^{92}$, F. Marroquim ${ }^{26 a}$, S.P. Marsden ${ }^{87}$, Z. Marshall ${ }^{16}$, M.U.F Martensson ${ }^{168}$, S. Marti-Garcia ${ }^{170}$, C.B. Martin ${ }^{113}$, T.A. Martin ${ }^{173}$, V.J. Martin ${ }^{49}$, B. Martin dit Latour ${ }^{15}$, M. Martinez ${ }^{13, v}$, V.I. Martinez Outschoorn ${ }^{169}$, S. Martin-Haugh ${ }^{133}$, V.S. Martoiu ${ }^{28 b}$, A.C. Martyniuk ${ }^{81}$, 


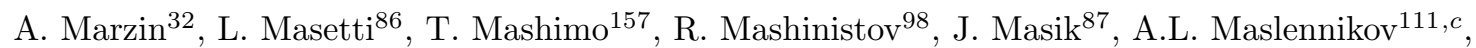
L. Massa ${ }^{135 a, 135 b}$, P. Mastrandrea ${ }^{5}$, A. Mastroberardino ${ }^{40 a, 40 b}$, T. Masubuchi ${ }^{157}$, P. Mättig ${ }^{178}$, J. Maurer ${ }^{28 b}$, S.J. Maxfield ${ }^{77}$, D.A. Maximov ${ }^{111, c}$, R. Mazini ${ }^{153}$, I. Maznas ${ }^{156}$, S.M. Mazza ${ }^{94 a, 94 b}$, N.C. Mc Fadden ${ }^{107}$, G. Mc Goldrick ${ }^{161}$, S.P. Mc Kee ${ }^{92}$, A. McCarn ${ }^{92}$, R.L. McCarthy ${ }^{150}$,

T.G. McCarthy ${ }^{103}$, L.I. McClymont ${ }^{81}$, E.F. McDonald ${ }^{91}$, J.A. Mcfayden ${ }^{32}$, G. Mchedlidze ${ }^{57}$, S.J. McMahon ${ }^{133}$, P.C. McNamara ${ }^{91}$, C.J. McNicol ${ }^{173}$, R.A. McPherson ${ }^{172, o}$, S. Meehan ${ }^{140}$, T.J. Megy $^{51}$, S. Mehlhase ${ }^{102}$, A. Mehta ${ }^{77}$, T. Meideck ${ }^{58}$, K. Meier ${ }^{60 a}$, B. Meirose ${ }^{44}$, D. Melini ${ }^{170, a i}$, B.R. Mellado Garcia ${ }^{147 c}$, J.D. Mellenthin ${ }^{57}$, M. Melo ${ }^{146 a}$, F. Meloni ${ }^{18}$,

A. Melzer ${ }^{23}$, S.B. Menary ${ }^{87}$, L. Meng ${ }^{77}$, X.T. Meng ${ }^{92}$, A. Mengarelli22a,22b, S. Menke ${ }^{103}$, E. Meoni ${ }^{40 a, 40 b}$, S. Mergelmeyer ${ }^{17}$, C. Merlassino ${ }^{18}$, P. Mermod $^{52}$, L. Merola ${ }^{106 a, 106 b}$, C. Meroni ${ }^{94 a}$, F.S. Merritt ${ }^{33}$, A. Messina ${ }^{134 a, 134 b}$, J. Metcalfe ${ }^{6}$, A.S. Mete ${ }^{166}$, C. Meyer ${ }^{124}$, J-P. Meyer ${ }^{138}$, J. Meyer ${ }^{109}$, H. Meyer Zu Theenhausen ${ }^{60 a}$, F. Miano ${ }^{151}$, R.P. Middleton ${ }^{133}$, S. Miglioranzi ${ }^{53 a, 53 b}$, L. Mijović ${ }^{49}$, G. Mikenberg ${ }^{175}$, M. Mikestikova ${ }^{129}$, M. Mikuž ${ }^{78}$, M. Milesi ${ }^{91}$, A. Milic ${ }^{161}$, D.A. Millar ${ }^{79}$, D.W. Miller ${ }^{33}$, C. Mills ${ }^{49}$, A. Milov ${ }^{175}$, D.A. Milstead ${ }^{148 a, 148 b}$, A.A. Minaenko ${ }^{132}$, Y. Minami ${ }^{157}$, I.A. Minashvili ${ }^{54 b}$, A.I. Mincer ${ }^{112}$, B. Mindur ${ }^{41 a}$, M. Mineev ${ }^{68}$, Y. Minegishi ${ }^{157}$, Y. Ming ${ }^{176}$, L.M. Mir ${ }^{13}$, K.P. Mistry ${ }^{124}$, T. Mitani ${ }^{174}$, J. Mitrevski ${ }^{102}$, V.A. Mitsou ${ }^{170}$, A. Miucci ${ }^{18}$, P.S. Miyagawa ${ }^{141}$, A. Mizukami ${ }^{69}$, J.U. Mjörnmark ${ }^{84}$, T. Mkrtchyan ${ }^{180}$, M. Mlynarikova ${ }^{131}$, T. Moa ${ }^{148 a, 148 b}$, K. Mochizuki ${ }^{97}$, P. Mogg ${ }^{51}$, S. Mohapatra ${ }^{38}$, S. Molander ${ }^{148 a, 148 b}$, R. Moles-Valls ${ }^{23}$, M.C. Mondragon ${ }^{93}$, K. Mönig ${ }^{45}$, J. Monk ${ }^{39}$, E. Monnier ${ }^{88}$, A. Montalbano ${ }^{150}$, J. Montejo Berlingen ${ }^{32}$, F. Monticelli ${ }^{74}$, S. Monzani ${ }^{94 a, 94 b}$, R.W. Moore ${ }^{3}$, N. Morange ${ }^{119}$, D. Moreno ${ }^{21}$, M. Moreno Llácer ${ }^{32}$, P. Morettini ${ }^{53 a}$, S. Morgenstern ${ }^{32}$, D. Mori ${ }^{144}$, T. Mori ${ }^{157}$, M. Morii ${ }^{59}$, M. Morinaga ${ }^{174}$, V. Morisbak ${ }^{121}$, A.K. Morley ${ }^{32}$, G. Mornacchi ${ }^{32}$, J.D. Morris ${ }^{79}$, L. Morvaj ${ }^{150}$, P. Moschovakos ${ }^{10}$, M. Mosidze ${ }^{54 b}$, H.J. Moss ${ }^{141}$, J. Moss ${ }^{145, a j}$, K. Motohashi ${ }^{159}$, R. Mount ${ }^{145}$, E. Mountricha ${ }^{27}$, E.J.W. Moyse ${ }^{89}$, S. Muanza ${ }^{88}$, F. Mueller ${ }^{103}$, J. Mueller ${ }^{127}$, R.S.P. Mueller ${ }^{102}$,

D. Muenstermann ${ }^{75}$, P. Mullen ${ }^{56}$, G.A. Mullier ${ }^{18}$, F.J. Munoz Sanchez ${ }^{87}$, W.J. Murray ${ }^{173,133}$, H. Musheghyan ${ }^{32}$, M. Muškinja ${ }^{78}$, A.G. Myagkov ${ }^{132, a k}$, M. Myska ${ }^{130}$, B.P. Nachman ${ }^{16}$, O. Nackenhorst ${ }^{52}$, K. Nagai ${ }^{122}$, R. Nagai ${ }^{69, a d}$, K. Nagano ${ }^{69}$, Y. Nagasaka ${ }^{61}$, K. Nagata ${ }^{164}$, M. Nagel ${ }^{51}$, E. Nagy ${ }^{88}$, A.M. Nairz ${ }^{32}$, Y. Nakahama ${ }^{105}$, K. Nakamura ${ }^{69}$, T. Nakamura ${ }^{157}$, I. Nakano ${ }^{114}$, R.F. Naranjo Garcia ${ }^{45}$, R. Narayan ${ }^{11}$, D.I. Narrias Villar ${ }^{60 a}$, I. Naryshkin ${ }^{125}$, T. Naumann ${ }^{45}$, G. Navarro ${ }^{21}$, R. Nayyar ${ }^{7}$, H.A. Neal ${ }^{92}$, P.Yu. Nechaeva ${ }^{98}$, T.J. Neep ${ }^{138}$, A. Negri ${ }^{123 a, 123 b}$, M. Negrini ${ }^{22 a}$, S. Nektarijevic ${ }^{108}$, C. Nellist ${ }^{119}$, A. Nelson ${ }^{166}$, M.E. Nelson ${ }^{122}$, S. Nemecek ${ }^{129}$, P. Nemethy ${ }^{112}$, M. Nessi ${ }^{32, a l}$, M.S. Neubauer ${ }^{169}$, M. Neumann ${ }^{178}$, P.R. Newman ${ }^{19}$, T.Y. Ng ${ }^{62 c}$, T. Nguyen Manh ${ }^{97}$, R.B. Nickerson ${ }^{122}$, R. Nicolaidou ${ }^{138}$, J. Nielsen ${ }^{139}$,

V. Nikolaenko ${ }^{132, a k}$, I. Nikolic-Audit ${ }^{83}$, K. Nikolopoulos ${ }^{19}$, J.K. Nilsen ${ }^{121}$, P. Nilsson ${ }^{27}$, Y. Ninomiya ${ }^{157}$, A. Nisati ${ }^{134 a}$, N. Nishu ${ }^{36 c}$, R. Nisius ${ }^{103}$, I. Nitsche ${ }^{46}$, T. Nitta ${ }^{174}$, T. Nobe $^{157}$, Y. Noguchi ${ }^{71}$, M. Nomachi ${ }^{120}$, I. Nomidis ${ }^{31}$, M.A. Nomura ${ }^{27}$, T. Nooney ${ }^{79}$, M. Nordberg ${ }^{32}$, N. Norjoharuddeen ${ }^{122}$, O. Novgorodova ${ }^{47}$, M. Nozaki ${ }^{69}$, L. Nozka ${ }^{117}$, K. Ntekas ${ }^{166}$, E. Nurse ${ }^{81}$, F. Nuti ${ }^{91}$, K. O'connor ${ }^{25}$, D.C. O'Neil ${ }^{144}$, A.A. O'Rourke ${ }^{45}$, V. O'Shea ${ }^{56}$, F.G. Oakham ${ }^{31, d}$, H. Oberlack ${ }^{103}$, T. Obermann ${ }^{23}$, J. Ocariz ${ }^{83}$, A. Ochi ${ }^{70}$, I. Ochoa ${ }^{38}$, J.P. Ochoa-Ricoux ${ }^{34 a}$, S. Oda ${ }^{73}$, S. Odaka ${ }^{69}$, A. Oh ${ }^{87}$, S.H. Oh ${ }^{48}$, C.C. Ohm ${ }^{16}$, H. Ohman ${ }^{168}$, H. Oide ${ }^{53 a, 53 b}$, H. Okawa ${ }^{164}$, Y. Okumura ${ }^{157}$, T. Okuyama ${ }^{69}$, A. Olariu ${ }^{28 b}$, L.F. Oleiro Seabra ${ }^{128 a}$, S.A. Olivares Pino ${ }^{34 a}$, D. Oliveira Damazio ${ }^{27}$, A. Olszewski ${ }^{42}$, J. Olszowska ${ }^{42}$, A. Onofre ${ }^{128 a, 128 e}$, K. Onogi ${ }^{105}$, P.U.E. Onyisi ${ }^{11, z}$, H. Oppen ${ }^{121}$, M.J. Oreglia ${ }^{33}$, Y. Oren ${ }^{155}$, D. Orestano ${ }^{136 a, 136 b}$, N. Orlando ${ }^{62 b}$, R.S. Orr ${ }^{161}$, B. Osculati ${ }^{53 a, 53 b, *}$, R. Ospanov ${ }^{36 a}$, G. Otero y Garzon ${ }^{29}$, H. Otono ${ }^{73}$, M. Ouchrif ${ }^{137 d}$, F. Ould-Saada ${ }^{121}$, A. Ouraou ${ }^{138}$, K.P. Oussoren ${ }^{109}$, Q. Ouyang ${ }^{35 a}$, M. Owen ${ }^{56}$, R.E. Owen ${ }^{19}$, V.E. Ozcan ${ }^{20 a}$, N. Ozturk ${ }^{8}$, K. Pachal ${ }^{144}$, A. Pacheco Pages ${ }^{13}$, L. Pacheco Rodriguez ${ }^{138}$, C. Padilla Aranda ${ }^{13}$, S. Pagan Griso ${ }^{16}$, M. Paganini ${ }^{179}$, F. Paige ${ }^{27}$, 
G. Palacino ${ }^{64}$, S. Palazzo ${ }^{40 a, 40 b}$, S. Palestini ${ }^{32}$, M. Palka ${ }^{41 b}$, D. Pallin ${ }^{37}$, E.St. Panagiotopoulou ${ }^{10}$, I. Panagoulias ${ }^{10}$, C.E. Pandini ${ }^{126 a, 126 b}$, J.G. Panduro Vazquez ${ }^{80}$, P. Pani ${ }^{32}$, S. Panitkin ${ }^{27}$,

D. Pantea ${ }^{28 b}$, L. Paolozzi ${ }^{52}$, Th.D. Papadopoulou ${ }^{10}$, K. Papageorgiou ${ }^{9, s}$, A. Paramonov ${ }^{6}$,

D. Paredes Hernandez ${ }^{179}$, A.J. Parker ${ }^{75}$, M.A. Parker ${ }^{30}$, K.A. Parker ${ }^{45}$, F. Parodi ${ }^{53 a, 53 b}$,

J.A. Parsons ${ }^{38}$, U. Parzefall ${ }^{51}$, V.R. Pascuzzi ${ }^{161}$, J.M. Pasner ${ }^{139}$, E. Pasqualucci ${ }^{134 a}$,

S. Passaggio ${ }^{53 a}$, Fr. Pastore ${ }^{80}$, S. Pataraia ${ }^{86}$, J.R. Pater ${ }^{87}$, T. Pauly ${ }^{32}$, B. Pearson ${ }^{103}$,

S. Pedraza Lopez ${ }^{170}$, R. Pedro ${ }^{128 a, 128 b}$, S.V. Peleganchuk ${ }^{111, c}$, O. Penc ${ }^{129}$, C. Peng ${ }^{35 a}$,

H. Peng ${ }^{36 a}$, J. Penwell ${ }^{64}$, B.S. Peralva ${ }^{26 b}$, M.M. Perego ${ }^{138}$, D.V. Perepelitsa ${ }^{27}$, F. Peri ${ }^{17}$,

L. Perini ${ }^{94 a, 94 b}$, H. Pernegger ${ }^{32}$, S. Perrella ${ }^{106 a, 106 b}$, R. Peschke ${ }^{45}$, V.D. Peshekhonov ${ }^{68, *}$,

K. Peters $^{45}$, R.F.Y. Peters ${ }^{87}$, B.A. Petersen $^{32}$, T.C. Petersen ${ }^{39}$, E. Petit ${ }^{58}$, A. Petridis ${ }^{1}$,

C. Petridou ${ }^{156}$, P. Petroff ${ }^{119}$, E. Petrolo ${ }^{134 a}$, M. Petrov ${ }^{122}$, F. Petrucci ${ }^{136 a, 136 b}$, N.E. Pettersson ${ }^{89}$,

A. Peyaud ${ }^{138}$, R. Pezoa ${ }^{34 b}$, F.H. Phillips ${ }^{93}$, P.W. Phillips ${ }^{133}$, G. Piacquadio ${ }^{150}$, E. Pianori ${ }^{173}$,

A. Picazio ${ }^{89}$, E. Piccaro ${ }^{79}$, M.A. Pickering ${ }^{122}$, R. Piegaia ${ }^{29}$, J.E. Pilcher ${ }^{33}$, A.D. Pilkington ${ }^{87}$,

A.W.J. Pin ${ }^{87}$, M. Pinamonti ${ }^{135 a, 135 b}$, J.L. Pinfold ${ }^{3}$, H. Pirumov ${ }^{45}$, M. Pitt ${ }^{175}$, L. Plazak ${ }^{146 a}$,

M.-A. Pleier ${ }^{27}$, V. Pleskot ${ }^{86}$, E. Plotnikova ${ }^{68}$, D. Pluth ${ }^{67}$, P. Podberezko ${ }^{111}$, R. Poettgen ${ }^{84}$,

R. Poggi ${ }^{123 a}$,123b, L. Poggioli ${ }^{119}$, I. Pogrebnyak ${ }^{93}$, D. Pohl ${ }^{23}$, G. Polesello ${ }^{123 a}$, A. Poley ${ }^{45}$,

A. Policicchio ${ }^{40 a, 40 b}$, R. Polifka ${ }^{32}$, A. Polini ${ }^{22 a}$, C.S. Pollard ${ }^{56}$, V. Polychronakos ${ }^{27}$, K. Pommès $^{32}$,

D. Ponomarenko ${ }^{100}$, L. Pontecorvo ${ }^{134 a}$, G.A. Popeneciu ${ }^{28 d}$, S. Pospisil ${ }^{130}$, K. Potamianos ${ }^{16}$,

I.N. Potrap ${ }^{68}$, C.J. Potter ${ }^{30}$, H. Potti ${ }^{11}$, T. Poulsen ${ }^{84}$, J. Poveda ${ }^{32}$, M.E. Pozo Astigarraga ${ }^{32}$,

P. Pralavorio ${ }^{88}$, A. Pranko ${ }^{16}$, S. Prell ${ }^{67}$, D. Price ${ }^{87}$, M. Primavera ${ }^{76 a}$, S. Prince ${ }^{90}$, N. Proklova ${ }^{100}$,

K. Prokofiev ${ }^{62 c}$, F. Prokoshin ${ }^{34 b}$, S. Protopopescu ${ }^{27}$, J. Proudfoot ${ }^{6}$, M. Przybycien ${ }^{41 a}$, A. Puri ${ }^{169}$,

P. Puzo ${ }^{119}$, J. Qian ${ }^{92}$, G. Qin ${ }^{56}$, Y. Qin ${ }^{87}$, A. Quadt ${ }^{57}$, M. Queitsch-Maitland ${ }^{45}$, D. Quilty ${ }^{56}$,

S. Raddum ${ }^{121}$, V. Radeka ${ }^{27}$, V. Radescu ${ }^{122}$, S.K. Radhakrishnan ${ }^{150}$, P. Radloff ${ }^{118}$, P. Rados ${ }^{91}$,

F. Ragusa ${ }^{94 a, 94 b}$, G. Rahal ${ }^{181}$, J.A. Raine ${ }^{87}$, S. Rajagopalan ${ }^{27}$, C. Rangel-Smith ${ }^{168}$, T. Rashid ${ }^{119}$,

S. Raspopov ${ }^{5}$, M.G. Ratti ${ }^{94 a, 94 b}$, D.M. Rauch ${ }^{45}$, F. Rauscher ${ }^{102}$, S. Rave ${ }^{86}$, I. Ravinovich ${ }^{175}$,

J.H. Rawling ${ }^{87}$, M. Raymond ${ }^{32}$, A.L. Read ${ }^{121}$, N.P. Readioff ${ }^{58}$, M. Reale ${ }^{76 a, 76 b}$,

D.M. Rebuzzi 123a,123b, A. Redelbach ${ }^{177}$, G. Redlinger ${ }^{27}$, R. Reece ${ }^{139}$, R.G. Reed ${ }^{147 c}$, K. Reeves ${ }^{44}$,

L. Rehnisch ${ }^{17}$, J. Reichert ${ }^{124}$, A. Reiss ${ }^{86}$, C. Rembser ${ }^{32}$, H. Ren ${ }^{35 a}$, M. Rescigno ${ }^{134 a}$,

S. Resconi ${ }^{94 a}$, E.D. Resseguie ${ }^{124}$, S. Rettie ${ }^{171}$, E. Reynolds ${ }^{19}$, O.L. Rezanova ${ }^{111, c}$, P. Reznicek ${ }^{131}$,

R. Rezvani ${ }^{97}$, R. Richter ${ }^{103}$, S. Richter ${ }^{81}$, E. Richter-Was ${ }^{41 b}$, O. Ricken ${ }^{23}$, M. Ridel ${ }^{83}$, P. Rieck ${ }^{103}$,

C.J. Riegel ${ }^{178}$, J. Rieger ${ }^{57}$, O. Rifki ${ }^{115}$, M. Rijssenbeek ${ }^{150}$, A. Rimoldi123a,123b, M. Rimoldi ${ }^{18}$,

L. Rinaldi ${ }^{22 a}$, G. Ripellino ${ }^{149}$, B. Ristic ${ }^{32}$, E. Ritsch ${ }^{32}$, I. Riu ${ }^{13}$, F. Rizatdinova ${ }^{116}$, E. Rizvi ${ }^{79}$,

C. Rizzi ${ }^{13}$, R.T. Roberts ${ }^{87}$, S.H. Robertson ${ }^{90, o}$, A. Robichaud-Veronneau ${ }^{90}$, D. Robinson ${ }^{30}$,

J.E.M. Robinson ${ }^{45}$, A. Robson ${ }^{56}$, E. Rocco ${ }^{86}$, C. Roda ${ }^{126 a, 126 b}$, Y. Rodina ${ }^{88, a m}$,

S. Rodriguez Bosca ${ }^{170}$, A. Rodriguez Perez ${ }^{13}$, D. Rodriguez Rodriguez ${ }^{170}$, S. Roe ${ }^{32}$, C.S. Rogan ${ }^{59}$,

O. Røhne ${ }^{121}$, J. Roloff ${ }^{59}$, A. Romaniouk ${ }^{100}$, M. Romano $22 \mathrm{a}, 22 \mathrm{~b}$, S.M. Romano Saez ${ }^{37}$,

E. Romero Adam ${ }^{170}$, N. Rompotis ${ }^{77}$, M. Ronzani ${ }^{51}$, L. Roos ${ }^{83}$, S. Rosati ${ }^{134 a}$, K. Rosbach ${ }^{51}$,

P. Rose ${ }^{139}$, N.-A. Rosien ${ }^{57}$, E. Rossi ${ }^{106 a, 106 b}$, L.P. Rossi ${ }^{53 a}$, J.H.N. Rosten ${ }^{30}$, R. Rosten ${ }^{140}$,

M. Rotaru ${ }^{28 b}$, J. Rothberg ${ }^{140}$, D. Rousseau ${ }^{119}$, A. Rozanov ${ }^{88}$, Y. Rozen ${ }^{154}$, X. Ruan ${ }^{147 c}$,

F. Rubbo ${ }^{145}$, F. Rühr ${ }^{51}$, A. Ruiz-Martinez ${ }^{31}$, Z. Rurikova ${ }^{51}$, N.A. Rusakovich ${ }^{68}$, H.L. Russell ${ }^{90}$, J.P. Rutherfoord ${ }^{7}$, N. Ruthmann ${ }^{32}$, Y.F. Ryabov ${ }^{125}$, M. Rybar ${ }^{169}$, G. Rybkin ${ }^{119}$, S. Ryu ${ }^{6}$,

A. Ryzhov ${ }^{132}$, G.F. Rzehorz ${ }^{57}$, A.F. Saavedra ${ }^{152}$, G. Sabato ${ }^{109}$, S. Sacerdoti ${ }^{29}$,

H.F-W. Sadrozinski ${ }^{139}$, R. Sadykov ${ }^{68}$, F. Safai Tehrani ${ }^{134 a}$, P. Saha ${ }^{110}$, M. Sahinsoy ${ }^{60 a}$,

M. Saimpert ${ }^{45}$, M. Saito ${ }^{157}$, T. Saito ${ }^{157}$, H. Sakamoto ${ }^{157}$, Y. Sakurai ${ }^{174}$, G. Salamanna ${ }^{136 a, 136 b}$,

J.E. Salazar Loyola ${ }^{34 b}$, D. Salek ${ }^{109}$, P.H. Sales De Bruin ${ }^{168}$, D. Salihagic ${ }^{103}$, A. Salnikov ${ }^{145}$,

J. Salt ${ }^{170}$, D. Salvatore ${ }^{40 a, 40 b}$, F. Salvatore ${ }^{151}$, A. Salvucci ${ }^{62 a, 62 b, 62 c}$, A. Salzburger ${ }^{32}$,

D. Sammel ${ }^{51}$, D. Sampsonidis ${ }^{156}$, D. Sampsonidou ${ }^{156}$, J. Sánchez ${ }^{170}$, V. Sanchez Martinez ${ }^{170}$,

A. Sanchez Pineda ${ }^{167 a, 167 c}$, H. Sandaker ${ }^{121}$, R.L. Sandbach ${ }^{79}$, C.O. Sander ${ }^{45}$, M. Sandhoff ${ }^{178}$, 
C. Sandoval ${ }^{21}$, D.P.C. Sankey ${ }^{133}$, M. Sannino ${ }^{53 a, 53 b}$, Y. Sano ${ }^{105}$, A. Sansoni ${ }^{50}$, C. Santoni ${ }^{37}$, H. Santos ${ }^{128 a}$, I. Santoyo Castillo ${ }^{151}$, A. Sapronov ${ }^{68}$, J.G. Saraiva ${ }^{128 a, 128 d}$, B. Sarrazin ${ }^{23}$, O. Sasaki ${ }^{69}$, K. Sato ${ }^{164}$, E. Sauvan ${ }^{5}$, G. Savage ${ }^{80}$, P. Savard ${ }^{161, d}$, N. Savic ${ }^{103}$, C. Sawyer ${ }^{133}$, L. Sawyer ${ }^{82, u}$, J. Saxon ${ }^{33}$, C. Sbarra ${ }^{22 a}$, A. Sbrizzi ${ }^{22 a, 22 b}$, T. Scanlon ${ }^{81}$, D.A. Scannicchio ${ }^{166}$, J. Schaarschmidt ${ }^{140}$, P. Schacht ${ }^{103}$, B.M. Schachtner ${ }^{102}$, D. Schaefer ${ }^{32}$, L. Schaefer ${ }^{124}$, R. Schaefer ${ }^{45}$, J. Schaeffer ${ }^{86}$, S. Schaepe ${ }^{23}$, S. Schaetzel ${ }^{60 b}$, U. Schäfer ${ }^{86}$, A.C. Schaffer ${ }^{119}$, D. Schaile ${ }^{102}$, R.D. Schamberger ${ }^{150}$, V.A. Schegelsky ${ }^{125}$, D. Scheirich ${ }^{131}$, M. Schernau ${ }^{166}$, C. Schiavi ${ }^{53 a, 53 b}$, S. Schier ${ }^{139}$, L.K. Schildgen ${ }^{23}$, C. Schillo ${ }^{51}$, M. Schioppa ${ }^{40 a, 40 b}$, S. Schlenker ${ }^{32}$, K.R. Schmidt-Sommerfeld ${ }^{103}$, K. Schmieden ${ }^{32}$, C. Schmitt ${ }^{86}$, S. Schmitt ${ }^{45}$, S. Schmitz ${ }^{86}$, U. Schnoor ${ }^{51}$, L. Schoeffel ${ }^{138}$, A. Schoening ${ }^{60 b}$, B.D. Schoenrock ${ }^{93}$, E. Schopf ${ }^{23}$, M. Schott ${ }^{86}$, J.F.P. Schouwenberg ${ }^{108}$, J. Schovancova ${ }^{32}$, S. Schramm ${ }^{52}$, N. Schuh ${ }^{86}$, A. Schulte ${ }^{86}$, M.J. Schultens ${ }^{23}$, H.-C. Schultz-Coulon ${ }^{60 a}$, H. Schulz ${ }^{17}$, M. Schumacher ${ }^{51}$, B.A. Schumm ${ }^{139}$, Ph. Schune ${ }^{138}$, A. Schwartzman ${ }^{145}$, T.A. Schwarz ${ }^{92}$, H. Schweiger ${ }^{87}$, Ph. Schwemling ${ }^{138}$, R. Schwienhorst ${ }^{93}$, J. Schwindling ${ }^{138}$, A. Sciandra ${ }^{23}$, G. Sciolla ${ }^{25}$, M. Scornajenghi ${ }^{40 a, 40 b}$, F. Scuri ${ }^{126 a, 126 b}$, F. Scutti ${ }^{91}$, J. Searcy ${ }^{92}$, P. Seema ${ }^{23}$, S.C. Seidel ${ }^{107}$, A. Seiden ${ }^{139}$, J.M. Seixas ${ }^{26 a}$, G. Sekhniaidze ${ }^{106 a}$, K. Sekhon ${ }^{92}$, S.J. Sekula ${ }^{43}$, N. Semprini-Cesari ${ }^{22 a, 22 b}$, S. Senkin ${ }^{37}$,

C. Serfon ${ }^{121}$, L. Serin ${ }^{119}$, L. Serkin ${ }^{167 a, 167 b}$, M. Sessa ${ }^{136 a, 136 b}$, R. Seuster ${ }^{172}$, H. Severini ${ }^{115}$, T. Sfiligoj ${ }^{78}$, F. Sforza ${ }^{165}$, A. Sfyrla ${ }^{52}$, E. Shabalina ${ }^{57}$, N.W. Shaikh148a,148b, L.Y. Shan ${ }^{35 a}$, R. Shang ${ }^{169}$, J.T. Shank ${ }^{24}$, M. Shapiro ${ }^{16}$, P.B. Shatalov ${ }^{99}$, K. Shaw ${ }^{167 a, 167 b}$, S.M. Shaw ${ }^{87}$, A. Shcherbakova ${ }^{148 a, 148 b}$, C.Y. Shehu ${ }^{151}$, Y. Shen ${ }^{115}$, N. Sherafati ${ }^{31}$, P. Sherwood ${ }^{81}$, L. Shi ${ }^{153, a n}$, S. Shimizu ${ }^{70}$, C.O. Shimmin ${ }^{179}$, M. Shimojima ${ }^{104}$, I.P.J. Shipsey ${ }^{122}$, S. Shirabe ${ }^{73}$, M. Shiyakova ${ }^{68, a o}$, J. Shlomi ${ }^{175}$, A. Shmeleva ${ }^{98}$, D. Shoaleh Saadi ${ }^{97}$, M.J. Shochet ${ }^{33}$, S. Shojaii ${ }^{94 a, 94 b}$, D.R. Shope ${ }^{115}$, S. Shrestha ${ }^{113}$, E. Shulga ${ }^{100}$, M.A. Shupe ${ }^{7}$, P. Sicho ${ }^{129}$, A.M. Sickles ${ }^{169}$, P.E. Sidebo ${ }^{149}$, E. Sideras Haddad ${ }^{147 c}$, O. Sidiropoulou ${ }^{177}$, A. Sidoti ${ }^{22 a, 22 b}$, F. Siegert ${ }^{47}$, Dj. Sijacki ${ }^{14}$, J. Silva ${ }^{128 a, 128 d}$, S.B. Silverstein ${ }^{148 a}$, V. Simak ${ }^{130}$, Lj. Simic ${ }^{14}$, S. Simion ${ }^{119}$, E. Simioni ${ }^{86}$, B. Simmons ${ }^{81}$, M. Simon ${ }^{86}$, P. Sinervo ${ }^{161}$, N.B. Sinev ${ }^{118}$, M. Sioli ${ }^{22 a, 22 b}$, G. Siragusa ${ }^{177}$, I. Siral ${ }^{92}$, S.Yu. Sivoklokov ${ }^{101}$, J. Sjölin 148a,148b, M.B. Skinner ${ }^{75}$, P. Skubic ${ }^{115}$, M. Slater ${ }^{19}$, T. Slavicek ${ }^{130}$, M. Slawinska ${ }^{42}$, K. Sliwa ${ }^{165}$, R. Slovak ${ }^{131}$, V. Smakhtin ${ }^{175}$, B.H. Smart ${ }^{5}$, J. Smiesko ${ }^{146 a}$, N. Smirnov ${ }^{100}$, S.Yu. Smirnov ${ }^{100}$, Y. Smirnov ${ }^{100}$, L.N. Smirnova ${ }^{101, a p}$, O. Smirnova ${ }^{84}$, J.W. Smith ${ }^{57}$, M.N.K. Smith ${ }^{38}$, R.W. Smith ${ }^{38}$, M. Smizanska ${ }^{75}$, K. Smolek ${ }^{130}$, A.A. Snesarev ${ }^{98}$, I.M. Snyder ${ }^{118}$, S. Snyder ${ }^{27}$, R. Sobie ${ }^{172, o}$ F. Socher ${ }^{47}$, A. Soffer ${ }^{155}$, A. Søgaard ${ }^{49}$, D.A. Soh ${ }^{153}$, G. Sokhrannyi ${ }^{78}$, C.A. Solans Sanchez ${ }^{32}$, M. Solar ${ }^{130}$, E.Yu. Soldatov ${ }^{100}$, U. Soldevila ${ }^{170}$, A.A. Solodkov ${ }^{132}$, A. Soloshenko 68 , O.V. Solovyanov ${ }^{132}$, V. Solovyev ${ }^{125}$, P. Sommer ${ }^{51}$, H. Son ${ }^{165}$, A. Sopczak ${ }^{130}$, D. Sosa ${ }^{60 b}$, C.L. Sotiropoulou ${ }^{126 a, 126 b}$, R. Soualah ${ }^{167 a, 167 c}$, A.M. Soukharev ${ }^{111, c}$, D. South ${ }^{45}$, B.C. Sowden ${ }^{80}$, S. Spagnolo ${ }^{76 a, 76 b}$, M. Spalla ${ }^{126 a, 126 b}$, M. Spangenberg ${ }^{173}$, F. Spanò ${ }^{80}$, D. Sperlich ${ }^{17}$,

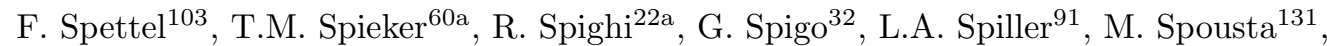
R.D. St. Denis ${ }^{56, *}$, A. Stabile ${ }^{94 a}$, R. Stamen ${ }^{60 a}$, S. Stamm ${ }^{17}$, E. Stanecka ${ }^{42}$, R.W. Stanek ${ }^{6}$, C. Stanescu ${ }^{136 a}$, M.M. Stanitzki ${ }^{45}$, B.S. Stapf ${ }^{109}$, S. Stapnes ${ }^{121}$, E.A. Starchenko ${ }^{132}$, G.H. Stark ${ }^{33}$, J. Stark ${ }^{58}$, S.H Stark ${ }^{39}$, P. Staroba ${ }^{129}$, P. Starovoitov ${ }^{60 a}$, S. Stärz ${ }^{32}$, R. Staszewski ${ }^{42}$, M. Stegler ${ }^{45}$, P. Steinberg ${ }^{27}$, B. Stelzer ${ }^{144}$, H.J. Stelzer ${ }^{32}$, O. Stelzer-Chilton ${ }^{163 a}$, H. Stenzel ${ }^{55}$, G.A. Stewart ${ }^{56}$, M.C. Stockton ${ }^{118}$, M. Stoebe ${ }^{90}$, G. Stoicea ${ }^{28 b}$, P. Stolte ${ }^{57}$, S. Stonjek ${ }^{103}$, A.R. Stradling ${ }^{8}$, A. Straessner ${ }^{47}$, M.E. Stramaglia ${ }^{18}$, J. Strandberg ${ }^{149}$, S. Strandberg ${ }^{148 a, 148 b}$, M. Strauss ${ }^{115}$, P. Strizenec ${ }^{146 b}$, R. Ströhmer ${ }^{177}$, D.M. Strom ${ }^{118}$, R. Stroynowski ${ }^{43}$, A. Strubig ${ }^{49}$, S.A. Stucci ${ }^{27}$, B. Stugu ${ }^{15}$, N.A. Styles ${ }^{45}$, D. $\mathrm{Su}^{145}$, J. Su${ }^{127}$, S. Suchek ${ }^{60 a}$, Y. Sugaya ${ }^{120}$, M. Suk ${ }^{130}$, V.V. Sulin ${ }^{98}$, DMS Sultan ${ }^{162 a, 162 b}$, S. Sultansoy ${ }^{4 c}$, T. Sumida ${ }^{71}$, S. Sun ${ }^{59}$, X. Sun ${ }^{3}$, K. Suruliz ${ }^{151}$, C.J.E. Suster ${ }^{152}$, M.R. Sutton ${ }^{151}$, S. Suzuki ${ }^{69}$, M. Svatos ${ }^{129}$, M. Swiatlowski ${ }^{33}$, S.P. Swift ${ }^{2}$,

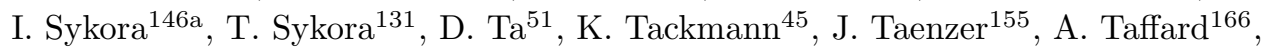


R. Tafirout ${ }^{163 a}$, E. Tahirovic ${ }^{79}$, N. Taiblum ${ }^{155}$, H. Takai ${ }^{27}$, R. Takashima ${ }^{72}$, E.H. Takasugi ${ }^{103}$, T. Takeshita ${ }^{142}$, Y. Takubo ${ }^{69}$, M. Talby ${ }^{88}$, A.A. Talyshev ${ }^{111, c}$, J. Tanaka ${ }^{157}$, M. Tanaka ${ }^{159}$, R. Tanaka ${ }^{119}$, S. Tanaka ${ }^{69}$, R. Tanioka ${ }^{70}$, B.B. Tannenwald ${ }^{113}$, S. Tapia Araya ${ }^{34 b}$, S. Tapprogge ${ }^{86}$, S. Tarem ${ }^{154}$, G.F. Tartarelli ${ }^{94 a}$, P. Tas ${ }^{131}$, M. Tasevsky ${ }^{129}$, T. Tashiro ${ }^{71}$, E. Tassi ${ }^{40 a, 40 b}$, A. Tavares Delgado ${ }^{128 a, 128 b}$, Y. Tayalati ${ }^{137 e}$, A.C. Taylor ${ }^{107}$, A.J. Taylor ${ }^{49}$, G.N. Taylor ${ }^{91}$, P.T.E. Taylor ${ }^{91}$, W. Taylor ${ }^{163 b}$, P. Teixeira-Dias ${ }^{80}$, D. Temple ${ }^{144}$, H. Ten Kate $^{32}$, P.K. Teng ${ }^{153}$,

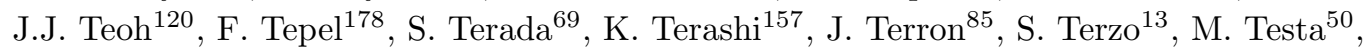
R.J. Teuscher ${ }^{161, o}$, T. Theveneaux-Pelzer ${ }^{88}$, F. Thiele ${ }^{39}$, J.P. Thomas ${ }^{19}$, J. Thomas-Wilsker ${ }^{80}$, P.D. Thompson ${ }^{19}$, A.S. Thompson ${ }^{56}$, L.A. Thomsen ${ }^{179}$, E. Thomson ${ }^{124}$, M.J. Tibbetts ${ }^{16}$, R.E. Ticse Torres ${ }^{88}$, V.O. Tikhomirov ${ }^{98, a q}$, Yu.A. Tikhonov ${ }^{111, c}$, S. Timoshenko ${ }^{100}$, P. Tipton ${ }^{179}$, S. Tisserant ${ }^{88}$, K. Todome ${ }^{159}$, S. Todorova-Nova ${ }^{5}$, S. Todt ${ }^{47}$, J. Tojo ${ }^{73}$, S. Tokár ${ }^{146 a}$, K. Tokushuku ${ }^{69}$, E. Tolley ${ }^{59}$, L. Tomlinson ${ }^{87}$, M. Tomoto ${ }^{105}$, L. Tompkins ${ }^{145}$,ar, K. Toms ${ }^{107}$, B. Tong ${ }^{59}$, P. Tornambe ${ }^{51}$, E. Torrence ${ }^{118}$, H. Torres ${ }^{47}$, E. Torró Pastor ${ }^{140}$, J. Toth ${ }^{88, a s}$,

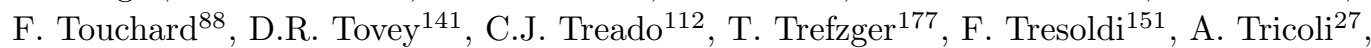
I.M. Trigger ${ }^{163 a}$, S. Trincaz-Duvoid ${ }^{83}$, M.F. Tripiana ${ }^{13}$, W. Trischuk ${ }^{161}$, B. Trocmé ${ }^{58}$, A. Trofymov ${ }^{45}$, C. Troncon ${ }^{94 a}$, M. Trottier-McDonald ${ }^{16}$, M. Trovatelli ${ }^{172}$, L. Truong ${ }^{147 b}$, M. Trzebinski ${ }^{42}$, A. Trzupek ${ }^{42}$, K.W. Tsang ${ }^{62 a}$, J.C-L. Tseng ${ }^{122}$, P.V. Tsiareshka ${ }^{95}$, G. Tsipolitis ${ }^{10}$, N. Tsirintanis ${ }^{9}$, S. Tsiskaridze ${ }^{13}$, V. Tsiskaridze ${ }^{51}$, E.G. Tskhadadze ${ }^{54 a}$, K.M. Tsui ${ }^{62 a}$, I.I. Tsukerman ${ }^{99}$, V. Tsulaia ${ }^{16}$, S. Tsuno ${ }^{69}$, D. Tsybychev ${ }^{150}$, Y. Tu ${ }^{62 b}$,

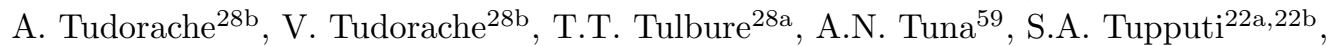
S. Turchikhin ${ }^{68}$, D. Turgeman ${ }^{175}$, I. Turk Cakir ${ }^{4 b, a t}$, R. Turra ${ }^{94 a}$, P.M. Tuts ${ }^{38}$, G. Ucchielli ${ }^{22 a, 22 b}$,

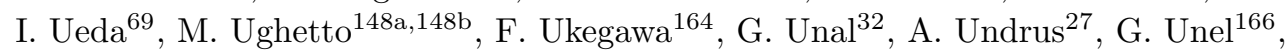
F.C. Ungaro ${ }^{91}$, Y. Unno ${ }^{69}$, C. Unverdorben ${ }^{102}$, J. Urban ${ }^{146 b}$, P. Urquijo ${ }^{91}$, P. Urrejola ${ }^{86}$,

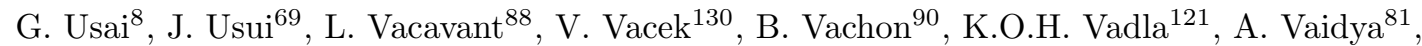
C. Valderanis ${ }^{102}$, E. Valdes Santurio ${ }^{148 a, 148 b}$, M. Valente ${ }^{52}$, S. Valentinetti ${ }^{22 a, 22 b}$, A. Valero ${ }^{170}$, L. Valéry ${ }^{13}$, S. Valkar ${ }^{131}$, A. Vallier ${ }^{5}$, J.A. Valls Ferrer ${ }^{170}$, W. Van Den Wollenberg ${ }^{109}$, H. van der Graaf ${ }^{109}$, P. van Gemmeren ${ }^{6}$, J. Van Nieuwkoop ${ }^{144}$, I. van Vulpen ${ }^{109}$, M.C. van Woerden ${ }^{109}$, M. Vanadia ${ }^{135 a, 135 b}$, W. Vandelli ${ }^{32}$, A. Vaniachine ${ }^{160}$, P. Vankov ${ }^{109}$,

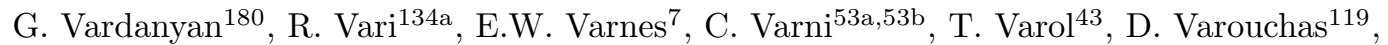
A. Vartapetian ${ }^{8}$, K.E. Varvell ${ }^{152}$, J.G. Vasquez ${ }^{179}$, G.A. Vasquez ${ }^{34 b}$, F. Vazeille ${ }^{37}$, D. Vazquez Furelos ${ }^{13}$, T. Vazquez Schroeder ${ }^{90}$, J. Veatch ${ }^{57}$, V. Veeraraghavan ${ }^{7}$, L.M. Veloce ${ }^{161}$, F. Veloso ${ }^{128 a, 128 c}$, S. Veneziano ${ }^{134 a}$, A. Ventura ${ }^{76 a, 76 b}$, M. Venturi ${ }^{172}$, N. Venturi ${ }^{32}$,

A. Venturini ${ }^{25}$, V. Vercesi ${ }^{123 a}$, M. Verducci ${ }^{136 a, 136 b}$, W. Verkerke ${ }^{109}$, A.T. Vermeulen ${ }^{109}$, J.C. Vermeulen ${ }^{109}$, M.C. Vetterli ${ }^{144, d}$, N. Viaux Maira ${ }^{34 b}$, O. Viazlo ${ }^{84}$, I. Vichou ${ }^{169, *}$, T. Vickey ${ }^{141}$, O.E. Vickey Boeriu ${ }^{141}$, G.H.A. Viehhauser ${ }^{122}$, S. Viel ${ }^{16}$, L. Vigani $^{122}$,

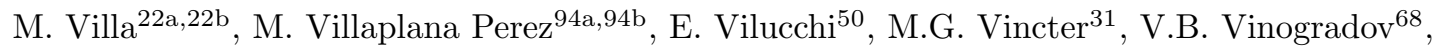

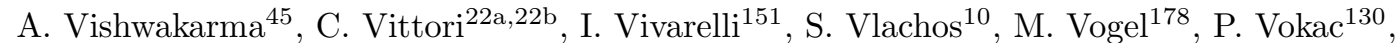
G. Volpi ${ }^{13}$, H. von der Schmitt ${ }^{103}$, E. von Toerne ${ }^{23}$, V. Vorobel ${ }^{131}$, K. Vorobev ${ }^{100}$, M. Vos $^{170}$, R. Voss $^{32}$, J.H. Vossebeld 77 , N. Vranjes ${ }^{14}$, M. Vranjes Milosavljevic ${ }^{14}$, V. Vrba ${ }^{130}$, M. Vreeswijk ${ }^{109}$, R. Vuillermet ${ }^{32}$, I. Vukotic ${ }^{33}$, P. Wagner ${ }^{23}$, W. Wagner ${ }^{178}$, J. Wagner-Kuhr ${ }^{102}$, H. Wahlberg ${ }^{74}$, S. Wahrmund ${ }^{47}$, J. Walder ${ }^{75}$, R. Walker ${ }^{102}$, W. Walkowiak ${ }^{143}$, V. Wallangen ${ }^{148 a, 148 b}$, C. Wang ${ }^{35 b}$, C. Wang ${ }^{36 b, a u}$, F. Wang ${ }^{176}$, H. Wang ${ }^{16}$, H. Wang ${ }^{3}$, J. Wang ${ }^{45}$, J. Wang ${ }^{152}$, Q. Wang ${ }^{115}$, R. Wang ${ }^{6}$, S.M. Wang ${ }^{153}$, T. Wang ${ }^{38}$, W. Wang ${ }^{153, a v}$,

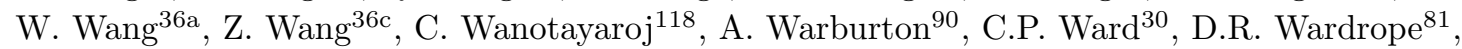
A. Washbrook ${ }^{49}$, P.M. Watkins ${ }^{19}$, A.T. Watson ${ }^{19}$, M.F. Watson ${ }^{19}$, G. Watts ${ }^{140}$, S. Watts ${ }^{87}$, B.M. Waugh ${ }^{81}$, A.F. Webb ${ }^{11}$, S. Webb ${ }^{86}$, M.S. Weber ${ }^{18}$, S.W. Weber ${ }^{177}$, S.A. Weber ${ }^{31}$, J.S. Webster ${ }^{6}$, A.R. Weidberg ${ }^{122}$, B. Weinert ${ }^{64}$, J. Weingarten ${ }^{57}$, M. Weirich $^{86}$, C. Weiser ${ }^{51}$,

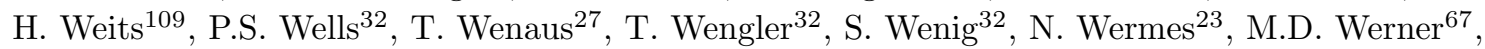


P. Werner ${ }^{32}$, M. Wessels ${ }^{60 a}$, T.D. Weston ${ }^{18}$, K. Whalen ${ }^{118}$, N.L. Whallon ${ }^{140}$, A.M. Wharton ${ }^{75}$, A.S. White ${ }^{92}$, A. White ${ }^{8}$, M.J. White ${ }^{1}$, R. White ${ }^{34 b}$, D. Whiteson ${ }^{166}$, B.W. Whitmore ${ }^{75}$, F.J. Wickens ${ }^{133}$, W. Wiedenmann ${ }^{176}$, M. Wielers ${ }^{133}$, C. Wiglesworth ${ }^{39}$, L.A.M. Wiik-Fuchs ${ }^{51}$, A. Wildauer ${ }^{103}$, F. Wilk ${ }^{87}$, H.G. Wilkens ${ }^{32}$, H.H. Williams ${ }^{124}$, S. Williams ${ }^{109}$, C. Willis ${ }^{93}$, S. Willocq ${ }^{89}$, J.A. Wilson ${ }^{19}$, I. Wingerter-Seez ${ }^{5}$, E. Winkels ${ }^{151}$, F. Winklmeier ${ }^{118}$, O.J. Winston ${ }^{151}$, B.T. Winter ${ }^{23}$, M. Wittgen ${ }^{145}$, M. Wobisch ${ }^{82, u}$, T.M.H. Wolf ${ }^{109}$, R. Wolff ${ }^{88}$, M.W. Wolter ${ }^{42}$, H. Wolters ${ }^{128 a, 128 c}$, V.W.S. Wong ${ }^{171}$, S.D. Worm ${ }^{19}$, B.K. Wosiek ${ }^{42}$, J. Wotschack ${ }^{32}$, K.W. Wozniak ${ }^{42}$, M. Wu ${ }^{33}$, S.L. $\mathrm{Wu}^{176}$, X. Wu ${ }^{52}$, Y. Wu ${ }^{92}$, T.R. Wyatt ${ }^{87}$, B.M. Wynne ${ }^{49}$, S. Xella ${ }^{39}$, Z. Xi ${ }^{92}$, L. Xia ${ }^{35 c}$, D. Xu ${ }^{35 a}$, L. Xu ${ }^{27}$, T. Xu ${ }^{138}$, B. Yabsley ${ }^{152}$, S. Yacoob ${ }^{147 a}$, D. Yamaguchi ${ }^{159}$, Y. Yamaguchi ${ }^{159}$, A. Yamamoto ${ }^{69}$, S. Yamamoto ${ }^{157}$,

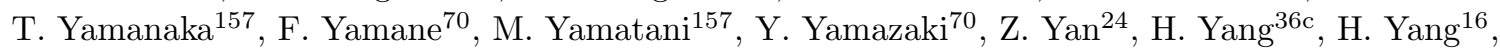
Y. Yang ${ }^{153}$, Z. Yang ${ }^{15}$, W-M. Yao ${ }^{16}$, Y.C. Yap ${ }^{83}$, Y. Yasu ${ }^{69}$, E. Yatsenko ${ }^{5}$, K.H. Yau Wong ${ }^{23}$,

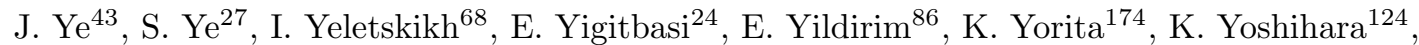
C. Young ${ }^{145}$, C.J.S. Young ${ }^{32}$, J. $\mathrm{Yu}^{8}$, J. Yu ${ }^{67}$, S.P.Y. Yuen ${ }^{23}$, I. Yusuff ${ }^{30, a w}$, B. Zabinski ${ }^{42}$, G. Zacharis ${ }^{10}$, R. Zaidan ${ }^{13}$, A.M. Zaitsev ${ }^{132, a k}$, N. Zakharchuk ${ }^{45}$, J. Zalieckas ${ }^{15}$, A. Zaman ${ }^{150}$, S. Zambito ${ }^{59}$, D. Zanzi ${ }^{11}$, C. Zeitnitz ${ }^{178}$, G. Zemaityte ${ }^{122}$, A. Zemla ${ }^{41 a}$, J.C. Zeng ${ }^{169}$, Q. Zeng ${ }^{145}$, O. Zenin ${ }^{132}$, T. Ženiš ${ }^{146 a}$, D. Zerwas ${ }^{119}$, D. Zhang ${ }^{92}$, F. Zhang ${ }^{176}$, G. Zhang ${ }^{36 a, a x}$, H. Zhang ${ }^{119}$, J. Zhang ${ }^{6}$, L. Zhang ${ }^{51}$, L. Zhang ${ }^{36 a}$, M. Zhang ${ }^{169}$, P. Zhang ${ }^{35 b}$, R. Zhang ${ }^{23}$, R. Zhang ${ }^{36 a, a u}$, X. Zhang ${ }^{36 b}$, Y. Zhang ${ }^{35 a}$, Z. Zhang ${ }^{119}$, X. Zhao ${ }^{43}$, Y. Zhao ${ }^{36 b, a y}$, Z. Zhao ${ }^{36 a}$, A. Zhemchugov ${ }^{68}$, B. Zhou ${ }^{92}$, C. Zhou ${ }^{176}$, L. Zhou ${ }^{43}$, M. Zhou ${ }^{35 a}$, M. Zhou ${ }^{150}$, N. Zhou ${ }^{35 c}$, C.G. Zhu ${ }^{36 b}, H^{2} Z^{2} u^{35 a}$, J. Zhu ${ }^{92}$, Y. Zhu ${ }^{36 a}$, X. Zhuang ${ }^{35 a}$, K. Zhukov ${ }^{98}$, A. Zibell ${ }^{177}$, D. Zieminska ${ }^{64}$, N.I. Zimine ${ }^{68}$, C. Zimmermann ${ }^{86}$, S. Zimmermann ${ }^{51}$, Z. Zinonos ${ }^{103}$, M. Zinser ${ }^{86}$, M. Ziolkowski ${ }^{143}$, L. Živković ${ }^{14}$, G. Zobernig ${ }^{176}$, A. Zoccoli ${ }^{22 a, 22 b}$, R. Zou ${ }^{33}$, M. zur Nedden ${ }^{17}$, L. Zwalinski ${ }^{32}$.

1 Department of Physics, University of Adelaide, Adelaide, Australia

2 Physics Department, SUNY Albany, Albany NY, United States of America

3 Department of Physics, University of Alberta, Edmonton AB, Canada

4 (a) Department of Physics, Ankara University, Ankara; ${ }^{(b)}$ Istanbul Aydin University, Istanbul;

(c) Division of Physics, TOBB University of Economics and Technology, Ankara, Turkey

5 LAPP, CNRS/IN2P3 and Université Savoie Mont Blanc, Annecy-le-Vieux, France

6 High Energy Physics Division, Argonne National Laboratory, Argonne IL, United States of America

7 Department of Physics, University of Arizona, Tucson AZ, United States of America

8 Department of Physics, The University of Texas at Arlington, Arlington TX, United States of America

9 Physics Department, National and Kapodistrian University of Athens, Athens, Greece

10 Physics Department, National Technical University of Athens, Zografou, Greece

11 Department of Physics, The University of Texas at Austin, Austin TX, United States of America

12 Institute of Physics, Azerbaijan Academy of Sciences, Baku, Azerbaijan

13 Institut de Física d'Altes Energies (IFAE), The Barcelona Institute of Science and Technology, Barcelona, Spain

14 Institute of Physics, University of Belgrade, Belgrade, Serbia

15 Department for Physics and Technology, University of Bergen, Bergen, Norway

16 Physics Division, Lawrence Berkeley National Laboratory and University of California, Berkeley CA, United States of America

17 Department of Physics, Humboldt University, Berlin, Germany

18 Albert Einstein Center for Fundamental Physics and Laboratory for High Energy Physics, University of Bern, Bern, Switzerland

19 School of Physics and Astronomy, University of Birmingham, Birmingham, United Kingdom

20 (a) Department of Physics, Bogazici University, Istanbul; ${ }^{(b)}$ Department of Physics Engineering, Gaziantep University, Gaziantep; ${ }^{(d)}$ Istanbul Bilgi University, Faculty of Engineering and Natural 
Sciences, Istanbul; ${ }^{(e)}$ Bahcesehir University, Faculty of Engineering and Natural Sciences, Istanbul, Turkey

21 Centro de Investigaciones, Universidad Antonio Narino, Bogota, Colombia

22 (a) INFN Sezione di Bologna; ${ }^{(b)}$ Dipartimento di Fisica e Astronomia, Università di Bologna, Bologna, Italy

23 Physikalisches Institut, University of Bonn, Bonn, Germany

24 Department of Physics, Boston University, Boston MA, United States of America

25 Department of Physics, Brandeis University, Waltham MA, United States of America

26 (a) Universidade Federal do Rio De Janeiro COPPE/EE/IF, Rio de Janeiro; ${ }^{(b)}$ Electrical Circuits Department, Federal University of Juiz de Fora (UFJF), Juiz de Fora; ${ }^{(c)}$ Federal University of Sao Joao del Rei (UFSJ), Sao Joao del Rei; ${ }^{(d)}$ Instituto de Fisica, Universidade de Sao Paulo, Sao Paulo, Brazil

27 Physics Department, Brookhaven National Laboratory, Upton NY, United States of America

28 (a) Transilvania University of Brasov, Brasov; ${ }^{(b)}$ Horia Hulubei National Institute of Physics and Nuclear Engineering, Bucharest; ${ }^{(c)}$ Department of Physics, Alexandru Ioan Cuza University of Iasi, Iasi; ${ }^{(d)}$ National Institute for Research and Development of Isotopic and Molecular Technologies, Physics Department, Cluj Napoca; ${ }^{(e)}$ University Politehnica Bucharest, Bucharest; (f) West University in Timisoara, Timisoara, Romania

29 Departamento de Física, Universidad de Buenos Aires, Buenos Aires, Argentina

30 Cavendish Laboratory, University of Cambridge, Cambridge, United Kingdom

31 Department of Physics, Carleton University, Ottawa ON, Canada

32 CERN, Geneva, Switzerland

33 Enrico Fermi Institute, University of Chicago, Chicago IL, United States of America

$34{ }^{(a)}$ Departamento de Física, Pontificia Universidad Católica de Chile, Santiago; ${ }^{(b)}$ Departamento de Física, Universidad Técnica Federico Santa María, Valparaíso, Chile

35 (a) Institute of High Energy Physics, Chinese Academy of Sciences, Beijing; ${ }^{(b)}$ Department of Physics, Nanjing University, Jiangsu; ${ }^{(c)}$ Physics Department, Tsinghua University, Beijing 100084, China

36 (a) Department of Modern Physics and State Key Laboratory of Particle Detection and Electronics, University of Science and Technology of China, Anhui; ${ }^{(b)}$ School of Physics, Shandong University, Shandong; ${ }^{(c)}$ Department of Physics and Astronomy, Key Laboratory for Particle Physics, Astrophysics and Cosmology, Ministry of Education; Shanghai Key Laboratory for Particle Physics and Cosmology, Shanghai Jiao Tong University, Shanghai(also at PKU-CHEP), China

37 Université Clermont Auvergne, CNRS/IN2P3, LPC, Clermont-Ferrand, France

38 Nevis Laboratory, Columbia University, Irvington NY, United States of America

39 Niels Bohr Institute, University of Copenhagen, Kobenhavn, Denmark

$40{ }^{(a)}$ INFN Gruppo Collegato di Cosenza, Laboratori Nazionali di Frascati; ${ }^{(b)}$ Dipartimento di Fisica, Università della Calabria, Rende, Italy

41 (a) AGH University of Science and Technology, Faculty of Physics and Applied Computer Science, Krakow; ${ }^{(b)}$ Marian Smoluchowski Institute of Physics, Jagiellonian University, Krakow, Poland

42 Institute of Nuclear Physics Polish Academy of Sciences, Krakow, Poland

43 Physics Department, Southern Methodist University, Dallas TX, United States of America

44 Physics Department, University of Texas at Dallas, Richardson TX, United States of America

45 DESY, Hamburg and Zeuthen, Germany

46 Lehrstuhl für Experimentelle Physik IV, Technische Universität Dortmund, Dortmund, Germany

47 Institut für Kern- und Teilchenphysik, Technische Universität Dresden, Dresden, Germany

48 Department of Physics, Duke University, Durham NC, United States of America

49 SUPA - School of Physics and Astronomy, University of Edinburgh, Edinburgh, United Kingdom

50 INFN e Laboratori Nazionali di Frascati, Frascati, Italy

51 Fakultät für Mathematik und Physik, Albert-Ludwigs-Universität, Freiburg, Germany

52 Departement de Physique Nucleaire et Corpusculaire, Université de Genève, Geneva, Switzerland

53 (a) INFN Sezione di Genova; ${ }^{(b)}$ Dipartimento di Fisica, Università di Genova, Genova, Italy 
54 (a) E. Andronikashvili Institute of Physics, Iv. Javakhishvili Tbilisi State University, Tbilisi;

(b) High Energy Physics Institute, Tbilisi State University, Tbilisi, Georgia

55 II Physikalisches Institut, Justus-Liebig-Universität Giessen, Giessen, Germany

56 SUPA - School of Physics and Astronomy, University of Glasgow, Glasgow, United Kingdom

57 II Physikalisches Institut, Georg-August-Universität, Göttingen, Germany

58 Laboratoire de Physique Subatomique et de Cosmologie, Université Grenoble-Alpes, CNRS/IN2P3, Grenoble, France

59 Laboratory for Particle Physics and Cosmology, Harvard University, Cambridge MA, United States of America

60 (a) Kirchhoff-Institut für Physik, Ruprecht-Karls-Universität Heidelberg, Heidelberg;

(b) Physikalisches Institut, Ruprecht-Karls-Universität Heidelberg, Heidelberg, Germany

61 Faculty of Applied Information Science, Hiroshima Institute of Technology, Hiroshima, Japan

62 (a) Department of Physics, The Chinese University of Hong Kong, Shatin, N.T., Hong Kong;

(b) Department of Physics, The University of Hong Kong, Hong Kong; ${ }^{(c)}$ Department of Physics and Institute for Advanced Study, The Hong Kong University of Science and Technology, Clear Water Bay, Kowloon, Hong Kong, China

63 Department of Physics, National Tsing Hua University, Taiwan, Taiwan

64 Department of Physics, Indiana University, Bloomington IN, United States of America

65 Institut für Astro- und Teilchenphysik, Leopold-Franzens-Universität, Innsbruck, Austria

66 University of Iowa, Iowa City IA, United States of America

67 Department of Physics and Astronomy, Iowa State University, Ames IA, United States of America

68 Joint Institute for Nuclear Research, JINR Dubna, Dubna, Russia

69 KEK, High Energy Accelerator Research Organization, Tsukuba, Japan

70 Graduate School of Science, Kobe University, Kobe, Japan

71 Faculty of Science, Kyoto University, Kyoto, Japan

72 Kyoto University of Education, Kyoto, Japan

73 Research Center for Advanced Particle Physics and Department of Physics, Kyushu University, Fukuoka, Japan

74 Instituto de Física La Plata, Universidad Nacional de La Plata and CONICET, La Plata, Argentina

75 Physics Department, Lancaster University, Lancaster, United Kingdom

$76{ }^{(a)}$ INFN Sezione di Lecce; ${ }^{(b)}$ Dipartimento di Matematica e Fisica, Università del Salento, Lecce, Italy

77 Oliver Lodge Laboratory, University of Liverpool, Liverpool, United Kingdom

78 Department of Experimental Particle Physics, Jožef Stefan Institute and Department of Physics, University of Ljubljana, Ljubljana, Slovenia

79 School of Physics and Astronomy, Queen Mary University of London, London, United Kingdom

80 Department of Physics, Royal Holloway University of London, Surrey, United Kingdom

81 Department of Physics and Astronomy, University College London, London, United Kingdom

82 Louisiana Tech University, Ruston LA, United States of America

83 Laboratoire de Physique Nucléaire et de Hautes Energies, UPMC and Université Paris-Diderot and CNRS/IN2P3, Paris, France

84 Fysiska institutionen, Lunds universitet, Lund, Sweden

85 Departamento de Fisica Teorica C-15, Universidad Autonoma de Madrid, Madrid, Spain

86 Institut für Physik, Universität Mainz, Mainz, Germany

87 School of Physics and Astronomy, University of Manchester, Manchester, United Kingdom

88 CPPM, Aix-Marseille Université and CNRS/IN2P3, Marseille, France

89 Department of Physics, University of Massachusetts, Amherst MA, United States of America

90 Department of Physics, McGill University, Montreal QC, Canada

91 School of Physics, University of Melbourne, Victoria, Australia

92 Department of Physics, The University of Michigan, Ann Arbor MI, United States of America

93 Department of Physics and Astronomy, Michigan State University, East Lansing MI, United States of America 
(a) INFN Sezione di Milano; ${ }^{(b)}$ Dipartimento di Fisica, Università di Milano, Milano, Italy

95 B.I. Stepanov Institute of Physics, National Academy of Sciences of Belarus, Minsk, Republic of Belarus

96 Research Institute for Nuclear Problems of Byelorussian State University, Minsk, Republic of Belarus

97 Group of Particle Physics, University of Montreal, Montreal QC, Canada

98 P.N. Lebedev Physical Institute of the Russian Academy of Sciences, Moscow, Russia

99 Institute for Theoretical and Experimental Physics (ITEP), Moscow, Russia

100 National Research Nuclear University MEPhI, Moscow, Russia

101 D.V. Skobeltsyn Institute of Nuclear Physics, M.V. Lomonosov Moscow State University, Moscow, Russia

102 Fakultät für Physik, Ludwig-Maximilians-Universität München, München, Germany

103 Max-Planck-Institut für Physik (Werner-Heisenberg-Institut), München, Germany

104 Nagasaki Institute of Applied Science, Nagasaki, Japan

105 Graduate School of Science and Kobayashi-Maskawa Institute, Nagoya University, Nagoya, Japan

$106{ }^{(a)}$ INFN Sezione di Napoli; ${ }^{(b)}$ Dipartimento di Fisica, Università di Napoli, Napoli, Italy

107 Department of Physics and Astronomy, University of New Mexico, Albuquerque NM, United States of America

108 Institute for Mathematics, Astrophysics and Particle Physics, Radboud University Nijmegen/Nikhef, Nijmegen, Netherlands

109 Nikhef National Institute for Subatomic Physics and University of Amsterdam, Amsterdam, Netherlands

110 Department of Physics, Northern Illinois University, DeKalb IL, United States of America

111 Budker Institute of Nuclear Physics, SB RAS, Novosibirsk, Russia

112 Department of Physics, New York University, New York NY, United States of America

113 Ohio State University, Columbus OH, United States of America

114 Faculty of Science, Okayama University, Okayama, Japan

115 Homer L. Dodge Department of Physics and Astronomy, University of Oklahoma, Norman OK, United States of America

116 Department of Physics, Oklahoma State University, Stillwater OK, United States of America

117 Palacký University, RCPTM, Olomouc, Czech Republic

118 Center for High Energy Physics, University of Oregon, Eugene OR, United States of America

119 LAL, Univ. Paris-Sud, CNRS/IN2P3, Université Paris-Saclay, Orsay, France

120 Graduate School of Science, Osaka University, Osaka, Japan

121 Department of Physics, University of Oslo, Oslo, Norway

122 Department of Physics, Oxford University, Oxford, United Kingdom

$123{ }^{(a)}$ INFN Sezione di Pavia; ${ }^{(b)}$ Dipartimento di Fisica, Università di Pavia, Pavia, Italy

124 Department of Physics, University of Pennsylvania, Philadelphia PA, United States of America

125 National Research Centre "Kurchatov Institute" B.P.Konstantinov Petersburg Nuclear Physics Institute, St. Petersburg, Russia

126 (a) INFN Sezione di Pisa; ${ }^{(b)}$ Dipartimento di Fisica E. Fermi, Università di Pisa, Pisa, Italy

127 Department of Physics and Astronomy, University of Pittsburgh, Pittsburgh PA, United States of America

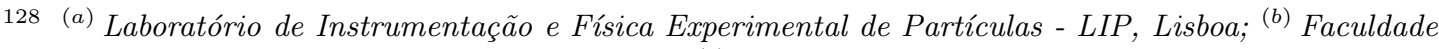
de Ciências, Universidade de Lisboa, Lisboa; ${ }^{(c)}$ Department of Physics, University of Coimbra, Coimbra; ${ }^{(d)}$ Centro de Física Nuclear da Universidade de Lisboa, Lisboa; ${ }^{(e)}$ Departamento de Fisica, Universidade do Minho, Braga; ${ }^{(f)}$ Departamento de Fisica Teorica y del Cosmos, Universidad de Granada, Granada; ${ }^{(g)}$ Dep Fisica and CEFITEC of Faculdade de Ciencias $e$ Tecnologia, Universidade Nova de Lisboa, Caparica, Portugal

129 Institute of Physics, Academy of Sciences of the Czech Republic, Praha, Czech Republic

130 Czech Technical University in Prague, Praha, Czech Republic

131 Charles University, Faculty of Mathematics and Physics, Prague, Czech Republic 
132 State Research Center Institute for High Energy Physics (Protvino), NRC KI, Russia

133 Particle Physics Department, Rutherford Appleton Laboratory, Didcot, United Kingdom

134 (a) INFN Sezione di Roma; ${ }^{(b)}$ Dipartimento di Fisica, Sapienza Università di Roma, Roma, Italy

$135{ }^{(a)}$ INFN Sezione di Roma Tor Vergata; ${ }^{(b)}$ Dipartimento di Fisica, Università di Roma Tor Vergata, Roma, Italy

136 (a) INFN Sezione di Roma Tre; ${ }^{(b)}$ Dipartimento di Matematica e Fisica, Università Roma Tre, Roma, Italy

137 (a) Faculté des Sciences Ain Chock, Réseau Universitaire de Physique des Hautes Energies Université Hassan II, Casablanca; ${ }^{(b)}$ Centre National de l'Energie des Sciences Techniques Nucleaires, Rabat; ${ }^{(c)}$ Faculté des Sciences Semlalia, Université Cadi Ayyad, LPHEA-Marrakech; ${ }^{(d)}$ Faculté des Sciences, Université Mohamed Premier and LPTPM, Oujda; ${ }^{(e)}$ Faculté des sciences, Université Mohammed V, Rabat, Morocco

138 DSM/IRFU (Institut de Recherches sur les Lois Fondamentales de l'Univers), CEA Saclay (Commissariat à l'Energie Atomique et aux Energies Alternatives), Gif-sur-Yvette, France

139 Santa Cruz Institute for Particle Physics, University of California Santa Cruz, Santa Cruz CA, United States of America

140 Department of Physics, University of Washington, Seattle WA, United States of America

141 Department of Physics and Astronomy, University of Sheffield, Sheffield, United Kingdom

142 Department of Physics, Shinshu University, Nagano, Japan

143 Department Physik, Universität Siegen, Siegen, Germany

144 Department of Physics, Simon Fraser University, Burnaby BC, Canada

145 SLAC National Accelerator Laboratory, Stanford CA, United States of America

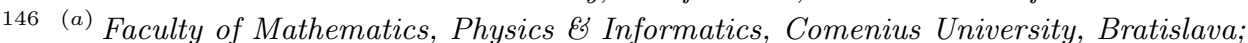

(b) Department of Subnuclear Physics, Institute of Experimental Physics of the Slovak Academy of Sciences, Kosice, Slovak Republic

147 (a) Department of Physics, University of Cape Town, Cape Town; ${ }^{(b)}$ Department of Physics, University of Johannesburg, Johannesburg; ${ }^{(c)}$ School of Physics, University of the Witwatersrand, Johannesburg, South Africa

$148{ }^{(a)}$ Department of Physics, Stockholm University; ${ }^{(b)}$ The Oskar Klein Centre, Stockholm, Sweden

149 Physics Department, Royal Institute of Technology, Stockholm, Sweden

150 Departments of Physics 65 Astronomy and Chemistry, Stony Brook University, Stony Brook NY, United States of America

151 Department of Physics and Astronomy, University of Sussex, Brighton, United Kingdom

152 School of Physics, University of Sydney, Sydney, Australia

153 Institute of Physics, Academia Sinica, Taipei, Taiwan

154 Department of Physics, Technion: Israel Institute of Technology, Haifa, Israel

155 Raymond and Beverly Sackler School of Physics and Astronomy, Tel Aviv University, Tel Aviv, Israel

156 Department of Physics, Aristotle University of Thessaloniki, Thessaloniki, Greece

157 International Center for Elementary Particle Physics and Department of Physics, The University of Tokyo, Tokyo, Japan

158 Graduate School of Science and Technology, Tokyo Metropolitan University, Tokyo, Japan

159 Department of Physics, Tokyo Institute of Technology, Tokyo, Japan

160 Tomsk State University, Tomsk, Russia

161 Department of Physics, University of Toronto, Toronto ON, Canada

$162{ }^{(a)}$ INFN-TIFPA; ${ }^{(b)}$ University of Trento, Trento, Italy

163 (a) TRIUMF, Vancouver BC; ${ }^{(b)}$ Department of Physics and Astronomy, York University, Toronto ON, Canada

164 Faculty of Pure and Applied Sciences, and Center for Integrated Research in Fundamental Science and Engineering, University of Tsukuba, Tsukuba, Japan

165 Department of Physics and Astronomy, Tufts University, Medford MA, United States of America 

America

$167{ }^{(a)}$ INFN Gruppo Collegato di Udine, Sezione di Trieste, Udine; ${ }^{(b)}$ ICTP, Trieste; ${ }^{(c)}$ Dipartimento di Chimica, Fisica e Ambiente, Università di Udine, Udine, Italy

168 Department of Physics and Astronomy, University of Uppsala, Uppsala, Sweden

169 Department of Physics, University of Illinois, Urbana IL, United States of America

170 Instituto de Fisica Corpuscular (IFIC), Centro Mixto Universidad de Valencia - CSIC, Spain

171 Department of Physics, University of British Columbia, Vancouver BC, Canada

172 Department of Physics and Astronomy, University of Victoria, Victoria BC, Canada

173 Department of Physics, University of Warwick, Coventry, United Kingdom

174 Waseda University, Tokyo, Japan

175 Department of Particle Physics, The Weizmann Institute of Science, Rehovot, Israel

176 Department of Physics, University of Wisconsin, Madison WI, United States of America

177 Fakultät für Physik und Astronomie, Julius-Maximilians-Universität, Würzburg, Germany

178 Fakultät für Mathematik und Naturwissenschaften, Fachgruppe Physik, Bergische Universität Wuppertal, Wuppertal, Germany

179 Department of Physics, Yale University, New Haven CT, United States of America

180 Yerevan Physics Institute, Yerevan, Armenia

181 Centre de Calcul de l'Institut National de Physique Nucléaire et de Physique des Particules (IN2P3), Villeurbanne, France

182 Academia Sinica Grid Computing, Institute of Physics, Academia Sinica, Taipei, Taiwan

a Also at Department of Physics, King's College London, London, United Kingdom

${ }^{b}$ Also at Institute of Physics, Azerbaijan Academy of Sciences, Baku, Azerbaijan

c Also at Novosibirsk State University, Novosibirsk, Russia

d Also at TRIUMF, Vancouver BC, Canada

e Also at Department of Physics 85 Astronomy, University of Louisville, Louisville, KY, United States of America

$f$ Also at Physics Department, An-Najah National University, Nablus, Palestine

$g$ Also at Department of Physics, California State University, Fresno CA, United States of America

$h$ Also at Department of Physics, University of Fribourg, Fribourg, Switzerland

${ }^{i}$ Also at II Physikalisches Institut, Georg-August-Universität, Göttingen, Germany

$j$ Also at Departament de Fisica de la Universitat Autonoma de Barcelona, Barcelona, Spain

${ }^{k}$ Also at Departamento de Fisica e Astronomia, Faculdade de Ciencias, Universidade do Porto, Portugal

${ }^{l}$ Also at Tomsk State University, Tomsk, and Moscow Institute of Physics and Technology State University, Dolgoprudny, Russia

${ }^{m}$ Also at The Collaborative Innovation Center of Quantum Matter (CICQM), Beijing, China

$n$ Also at Universita di Napoli Parthenope, Napoli, Italy

- Also at Institute of Particle Physics (IPP), Canada

${ }^{p}$ Also at Horia Hulubei National Institute of Physics and Nuclear Engineering, Bucharest, Romania

$q$ Also at Department of Physics, St. Petersburg State Polytechnical University, St. Petersburg, Russia

$r$ Also at Borough of Manhattan Community College, City University of New York, New York City, United States of America

$s$ Also at Department of Financial and Management Engineering, University of the Aegean, Chios, Greece

${ }^{t}$ Also at Centre for High Performance Computing, CSIR Campus, Rosebank, Cape Town, South Africa

${ }^{u}$ Also at Louisiana Tech University, Ruston LA, United States of America

$v$ Also at Institucio Catalana de Recerca $i$ Estudis Avancats, ICREA, Barcelona, Spain

$w$ Also at Graduate School of Science, Osaka University, Osaka, Japan 
$x$ Also at Fakultät für Mathematik und Physik, Albert-Ludwigs-Universität, Freiburg, Germany

y Also at Institute for Mathematics, Astrophysics and Particle Physics, Radboud University Nijmegen/Nikhef, Nijmegen, Netherlands

$z$ Also at Department of Physics, The University of Texas at Austin, Austin TX, United States of America

aa Also at Institute of Theoretical Physics, Ilia State University, Tbilisi, Georgia

ab Also at CERN, Geneva, Switzerland

ac Also at Georgian Technical University (GTU), Tbilisi, Georgia

ad Also at Ochadai Academic Production, Ochanomizu University, Tokyo, Japan

ae Also at Manhattan College, New York NY, United States of America

af Also at Departamento de Física, Pontificia Universidad Católica de Chile, Santiago, Chile

ag Also at Department of Physics, The University of Michigan, Ann Arbor MI, United States of America

ah Also at The City College of New York, New York NY, United States of America

ai Also at Departamento de Fisica Teorica y del Cosmos, Universidad de Granada, Granada, Portugal

aj Also at Department of Physics, California State University, Sacramento CA, United States of America

ak Also at Moscow Institute of Physics and Technology State University, Dolgoprudny, Russia

al Also at Departement de Physique Nucleaire et Corpusculaire, Université de Genève, Geneva, Switzerland

am Also at Institut de Fisica d'Altes Energies (IFAE), The Barcelona Institute of Science and Technology, Barcelona, Spain

an Also at School of Physics, Sun Yat-sen University, Guangzhou, China

ao Also at Institute for Nuclear Research and Nuclear Energy (INRNE) of the Bulgarian Academy of Sciences, Sofia, Bulgaria

ap Also at Faculty of Physics, M.V.Lomonosov Moscow State University, Moscow, Russia

aq Also at National Research Nuclear University MEPhI, Moscow, Russia

ar Also at Department of Physics, Stanford University, Stanford CA, United States of America

as Also at Institute for Particle and Nuclear Physics, Wigner Research Centre for Physics, Budapest, Hungary

at Also at Giresun University, Faculty of Engineering, Turkey

au Also at CPPM, Aix-Marseille Université and CNRS/IN2P3, Marseille, France

av Also at Department of Physics, Nanjing University, Jiangsu, China

aw Also at University of Malaya, Department of Physics, Kuala Lumpur, Malaysia

ax Also at Institute of Physics, Academia Sinica, Taipei, Taiwan

ay Also at LAL, Univ. Paris-Sud, CNRS/IN2P3, Université Paris-Saclay, Orsay, France

* Deceased 